\title{
Summary of the Third AIAA CFD Drag Prediction Workshop
}

\author{
John C. Vassberg*, Edward N. Tinoco†, Mori Mani ${ }^{\ddagger}$ \\ The Boeing Company, Huntington Beach, CA 92647, Seattle, WA, 98124, St. Louis, MO, 63301, USA
}

Olaf P. Brodersen, Bernhard Eisfeld ${ }^{\S}$

DLR Institute of Aerodynamics and Flow Technology, 38108 Braunschweig, Germany

\author{
Richard A. Wahls, Joseph H. Morrison" \\ NASA Langley Research Center, Hampton, VA 23681, USA \\ Tom Zickuhr,* Kelly R. Laflin ${ }^{\dagger \dagger}$ \\ Cessna Aircraft Company, Wichita, KS 67218, USA \\ Dimitri J. Mavriplis ${ }^{\ddagger \ddagger}$ \\ University of Wyoming, Laramie, WY 82071, USA
}

\begin{abstract}
Results from the Third AIAA Drag Prediction Workshop (DPW-III) are summarized. The workshop focused on the prediction of both absolute and differential drag levels for wingbody and wing-alone configurations that are representative of transonic transport aircraft. The baseline DLR-F6 wing-body geometry, previously utilized in DPW-II, is also augmented with a side-of-body fairing to help reduce the complexity of the flow physics in the wingbody juncture region. In addition, two new wing-alone geometries have been developed for DPW-III. Numerical calculations are performed using industry-relevant test cases that include lift-specific and fixed-alpha flight conditions, as well as full drag polars. Drag, lift and pitching moment predictions from numerous Reynolds-Averaged Navier-Stokes computational fluid dynamics methods are presented, focused on fully-turbulent flows. Solutions are performed on structured, unstructured, and hybrid grid systems. The structured grid sets include pointmatched multi-block meshes and over-set grid systems. The unstructured and hybrid grid sets are comprised of tetrahedral, pyramid, and prismatic elements. Effort was made to provide a high-quality and parametrically consistent family of grids for each grid type about each configuration under study. The wing-body families are comprised of a coarse, medium, and fine grid, while the wing-alone families also include an extra-fine mesh. These mesh sequences are utilized to help determine how the provided flow solutions fair with respect to asymptotic grid convergence, and are used to estimate an absolute drag of each configuration.
\end{abstract}

\footnotetext{
${ }^{*}$ Boeing Technical Fellow, AIAA Associate Fellow, DPW Chairman \& Charter Member

$\dagger$ Boeing Technical Fellow, AIAA Associate Fellow

$\ddagger_{B}$ Boeing Technical Fellow, AIAA Associate Fellow

$\S$ Research Engineer

I Assistant Head, Configuration Aerodynamics Branch, AIAA Associate Fellow, DPW Charter Member

$\|_{\text {Research Scientist, AIAA Senior Member }}$

** Senior Specialist Engineer, AIAA Senior Member, DPW Charter Member

$\dagger^{\dagger}$ Senior Specialist Engineer, AIAA Senior Member

$\ddagger \ddagger$ Professor Mechanical Engineering, AIAA Associate Fellow
} 


\section{Nomenclature}

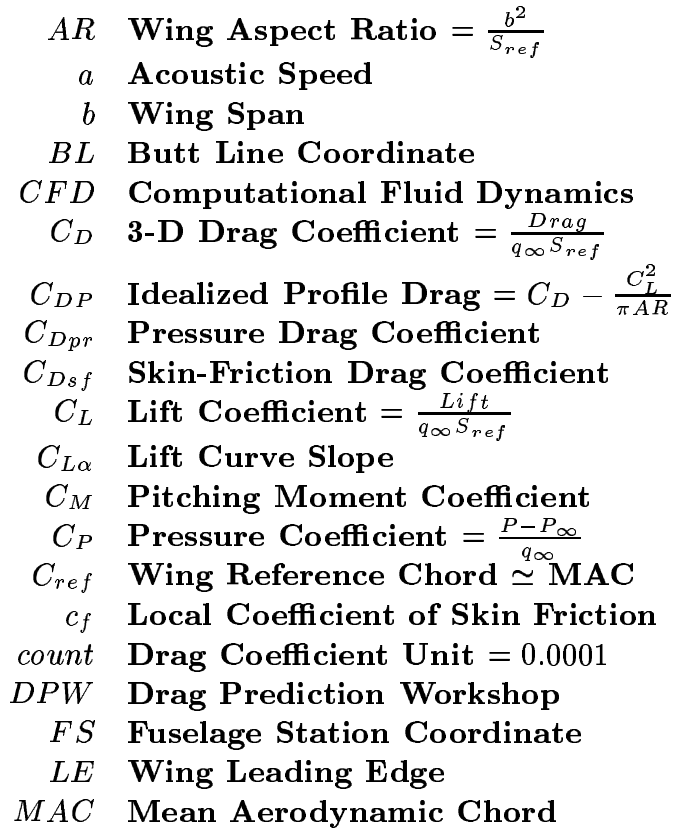

\author{
$N$ Total Number of Grid Points \\ $P$ Static Pressure \\ $q$ Dynamic Pressure $=\frac{1}{2} \rho V^{2}$ \\ $R A N S$ Reynolds-Averaged Navier-Stokes \\ $R e$ Reynolds number $=\frac{\rho_{\infty} V_{\infty} C_{r e f}}{\mu_{\infty}}$ \\ $S_{\text {ref }}$ Reference Area \\ $S O B$ Side-of-Body \\ $T$ Temperature \\ $T E$ Wing Trailing Edge \\ $V$ Velocity \\ $W B$ Wing/Body \\ $W B N P$ Wing/Body/Nacelle/Pylon \\ $W L$ Water Line Coordinate \\ $Y^{+} \quad$ Wall Distance $=R e \sqrt{\frac{c_{f}}{2}} y$ \\ $\alpha$ Angle of Attack \\ $\Delta$ Difference in Quantity \\ $\eta$ Fraction of Wing Semi-Span \\ $\mu$ Fluid Viscosity \\ $\rho$ Fluid Density \\ $\pi \quad 3.141592654 \ldots$ \\ $\infty$ Signifies Freestream Conditions
}

\section{Introduction}

The AIAA CFD Drag Prediction Workshop (DPW) Series was initiated by a working group of members from the AIAA Applied Aerodynamics Technical Committee. From the onset, the DPW organizing committee defined and has adhered to a set of primary objectives for the DPW Series. These include:

- Assess state-of-the-art computational fluid dynamics (CFD) methods as practical aerodynamic tools for the prediction of forces and moments on industry-relevant geometries, with a focus on absolute drag.

- Provide an impartial international forum for evaluating the effectiveness of CFD Navier-Stokes solvers.

- Promote balanced participation across academia, government labs, and industry.

- Use common public-domain subject geometries, simple enough to permit high-fidelity computations.

- Provide baseline grids to encourage participation and help reduce variability of CFD results.

- Openly discuss and identify areas needing additional research and development.

- Conduct rigorous statistical analyses of CFD results to establish confidence levels in predictions.

- Schedule open-forum sessions to further engage interaction among all interested parties.

- Maintain a public-domain accessible database of geometries, grids, and results.

- Document workshop findings; disseminate this information through publications and presentations.

The first workshop ${ }^{1}$ in this series, DPW-I, was held in Anaheim, CA in conjunction with the $19^{\text {th }}$ Applied Aerodynamics Conference of June 2001. The premise of DPW-I was to solicit CFD predictions of a common, industry relevant geometry and assess the results using statistical analysis techniques. Although the focus of the workshop was on drag prediction, lift and pitching moment predictions were also evaluated. The DLR-F4 wing-body configuration was chosen as the subject of DPW-I both because of its simplicity and the availability of publicly released experimental test data. ${ }^{2}$ The workshop committee provided a standard set of multi-block structured, overset, and unstructured grids for the DLR-F4 geometry to encourage participation in the workshop and reduce variability in the CFD results. However, participants were also encouraged to construct their own grids using their best practices so that learned knowledge concerning grid generation 
and drag prediction might be shared ${ }^{3}$ among workshop attendees. The test cases were chosen to reflect the interests of industry and included a fixed- $C_{L}$ single point solution, drag polar, and constant- $C_{L}$ drag rise data sets. Eighteen participants submitted results, using 14 different CFD codes; many submitted multiple sets of data exercising different options in their codes, e.g., turbulence models and/or different grids. A summary of these results was documented by the DPW-I organizing committee. ${ }^{4,5}$ Because of strong participation, DPW-I successfully amassed a CFD data set suitable for statistical analysis. ${ }^{6,7}$ However, the results of that analysis were rather disappointing, showing a 270-drag-count spread in the fixed- $C_{L}$ data, with a 100:1 confidence interval of more than \pm 50 drag counts.

Despite the disheartening results of the statistical analysis, DPW-I was a definitive success. It brought together CFD developers and practitioners and focused their efforts on a common problem. It facilitated an exchange of learned best practices and promoted open discussions, identifying areas requiring further research or additional scrutiny. Possibly most significant, it employed statistical methods to objectively assess CFD results. Finally, it reminded the CFD and applied aerodynamics communities that CFD is not yet a fully matured discipline.

In addition to the accomplishments listed above, DPW-I initiated interest in industry-relevant drag predictions that has been sustained through two more workshops, and looks to continue beyond. Several of the participants presented their DPW-I results ${ }^{8-12}$ at a well-attended special session of the 2002 AIAA Aerospace Sciences Meeting and Exhibit in Reno, NV. The interest generated by DPW-I naturally led to the planning and organization of the $2^{\text {nd }}$ AIAA Drag Prediction Workshop, DPW-II. The DPW-II organizing committee, recognizing the success of DPW-I, maintained its objectives for DPW-II.

The second workshop ${ }^{13}$ was held in Orlando, FL in conjunction with the $21^{\text {st }}$ Applied Aerodynamics Conference of June 2003. For this workshop, the DLR-F6 was chosen as the subject geometry, in both wing-body (WB) and wing-body-nacelle-pylon (WBNP) form. The DPW-II organizing committee worked with DLR and ONERA to make pertinent experimental data available to the public domain. One specific objective of DPW-II was the prediction of the incremental drag associated with nacelle/pylon installation. Although the F6 geometry is similar to that of the F4, its pockets of flow separation at the design condition are more severe; these occur predominantly at the wing/body and wing/pylon juncture regions. Again, this workshop was documented with a summary paper, ${ }^{14,15}$ a statistical analysis, ${ }^{16}$ an invited reflections paper ${ }^{17}$ on the workshop series, and numerous participant papers ${ }^{18-30}$ in two special sessions of the 2004 AIAA Aerospace Sciences Meeting in Reno, NV. A conclusion of DPW-II was that the separated flow regions made it difficult to draw meaningful conclusions with respect to grid convergence and drag prediction. During the follow-up open-forum discussions, the CFD community voiced the desire for the organizing committee to include in the third workshop: a) Blind Test Cases, and b) Simpler Geometries. The request for blind test cases is motivated by an earnest attempt to better establish a measure of the CFD community's capability to predict absolute drag, rather than match it after-the-fact. The request for simpler geometries allows more extensive research in studies of asymptotic grid convergence.

The third workshop ${ }^{31}$ was held in San Fransisco, CA in conjunction with the $24^{\text {th }}$ Applied Aerodynamics Conference of June 2006. The DLR-F6 WB from DPW-II was retained as a baseline configuration for the DPW-III to provide a bridge between these two workshops. However, to test the hypothesis that the grid-convergence issues of DPW-II were the direct result of the large pockets of flow separation, a new wing-body fairing was designed to eliminate the side-of-body separation. Details of the FX2B fairing design are documented by Vassberg et. al. ${ }^{32}$ In addition, to help reduce the wing upper-surface trailingedge flow separation, a higher Reynolds number was introduced for the WB test cases. These changes in both geometry and flow condition also provided the DPW-III participants a blind test since no test data would be available prior to the workshop. Furthermore, two wing-alone geometries were created to provide workshop participants with simpler configurations on which more extensive grid-convergence studies could be conducted; these wings were designed to not exhibit any appreciable separation at their design conditions.

The DPW-III had a total of 15 participants submit multiple datasets for the DLR-F6 WB cases and 10 participants submit datasets for the wing-alone cases. To illustrate the balance of participation achieved in these workshops, the demographics of the DPW-III breakdown follows.

- USA: $66 \%$, Europe: $21 \%$, Asia: $13 \%$

- Industry: 54\%, Government Labs: $33 \%$, Academia: $13 \%$

- Structured: $42 \%$, Hybrid: $46 \%$, Tetrahedra: $12 \%$

- Returning from DPW-II: $54 \%$, New to DPW: $46 \%$ 
In addition to the publications spawned directly by the DPW Series, the DPW databases have been used elsewhere and continue to be downloaded from the website. Two notable references are by Baker ${ }^{33}$ and Salas ${ }^{34}$ both provide independent, rigorous analyses of the grid-sensitivity data generated by the DPW-II. The conclusions of these studies were leveraged by the organizing committee to better construct the test cases for DPW-III, and although the applications of the test cases still have flaws, the lessons learned from each workshop have improved the outcome of subsequent workshops.

When the concept of this workshop series first began to take form in January of 2000, it was impossible then to imagine the magnitude of the cummulative efforts the DPW participants would be willing to invest. Even in retrospect, this is hard to believe. It is a testament that a grass-roots campaign such as this workshop series can accomplish so much. Through the contributions of the DPW participants, the public now has access to a wealth of previously-unavailable CFD data.

This paper is organized in the following manner. Section II provides a description of the subject configurations. Section III outlines the test cases of the third workshop. Section IV gives a brief description of the family of baseline grids utilized in the workshop. Section V summarizes the collective results of the DPW-III. Section VI provides a status of the wind-tunnel test campaign currently under work to collect experimental data on the DLR-F6 and FX2B WB configurations. Tables of data are embedded within the text closely after first reference, while all figures are appended to the end of this publication.

\section{Geometry Descriptions}

\section{II.A. DLR-F6 Wing/Body and FX2B Fairing}

The baseline WB configuration for DPW-III was the DLR-F6 wing/body which was the same geometry used as the test case in DPW-II. The F6 wind tunnel model represents an aircraft designed to cruise at transonic speeds with a wing leading-edge sweep of $27.1^{\circ}$ and a quarter-chord sweep of $25^{\circ}$. The dihedral of the wing is $4.787^{\circ}$. More reference quantities for this model are provided below.

$\begin{array}{rlrl}S_{r e f} / 2 & 72,700.0 \mathrm{~mm}^{2} & X_{r e f} & 157.9 \mathrm{~mm} \\ C_{r e f} & 141.2 \mathrm{~mm} & Y_{\text {ref }} & 0.0 \mathrm{~mm} \\ b / 2 & 585.647 \mathrm{~mm} & Z_{r e f} & -33.92 \mathrm{~mm}\end{array}$

Note that an aspect ratio of 9.5 is used herein instead of the computed value of 9.436 . The purpose of this is to remain consistent with the literature on this geometry. A planform view of the F6 WB configuration is shown in Figure 1. For more detailed information on this geometry, see Brodersen ${ }^{35}$ and Laflin. ${ }^{14}$

The DLR-F6 model has an acute angle, roughly $60^{\circ}$, between the fuselage and wing upper-surface at the trailing-edge which contributes or leads to flow separation at the WB juncture. A new WB fairing was developed by Vassberg et. al..$^{32}$ with the goal of completely removing the separation bubble present at the design conditions. For consideration of a possible follow-on wind-tunnel test, the only design constraint for this fairing was that it does not cut into the baseline DLR-F6 geometry. Further, no attempt was made to optimize drag reduction. By removing the side-of-body pocket of flow separation, this workshop could test the hypothesis that the complexity associated with flow separations is the root cause of the poor asymptotic grid convergence identified in DPW-II.

\section{II.B. DPW Wings W1 and W2}

Two wing-alone geometries DPW-W1/W2 were developed for DPW-III. Both have a common simple trapezoidal planform shape with no leading-edge or trailing-edge breaks. Their leading-edge sweep is $17.2^{\circ}$ and they have a quarter-chord sweep of $15.0^{\circ}$. The following summarizes their reference quantities.

$\begin{array}{rl}S_{r e f} / 2 & 145,161.0 \mathrm{~mm}^{2} \\ C_{r e f} & 197.556 \mathrm{~mm} \\ b / 2 & 762.0 \mathrm{~mm} \\ A R & 8.0\end{array}$

$W 1$

$X_{\text {ref }} \quad 6847.145 \mathrm{~mm}$

$Y_{\text {ref }} \quad 0.0 \mathrm{~mm}$

$Z_{\text {ref }} \quad 2226.615 \mathrm{~mm}$
$W 2$

$X_{\text {ref }} \quad 154.245 \mathrm{~mm}$

$Y_{\text {ref }} \quad 0.0 \mathrm{~mm}$

$Z_{\text {ref }} \quad 0.0 \mathrm{~mm}$

The airfoil selected for DPW-W1 is representative of a supercritical section found on most modern transport aircraft. At the design condition of Mach 0.76 and $C_{L}=0.50$, the upper surface pressure distribution 
has a nearly flat, roof-top shape with a shock located at about $57 \% x / c$. The second wing, DPW-W2, was created by optimizing the first.

A Tranair ${ }^{36}$ single-point optimization at 0.76 Mach and 5 million Reynolds number was performed on the DPW-W1 wing to create a test for drag increment prediction. Since the goal was to preserve the planform and thickness of the original wing, only camber and twist design variables were utilized. The variables were laid out at 7 spanwise cuts (airfoils), with 5 camber and 1 twist variable per airfoil, for a total of 42 geometry degrees of freedom. The twist variable rotates the airfoil about its trailing edge in a plane orthogonal to the y-axis. In addition, the angle of attack was allowed to vary in order to maintain the same lift coefficient at this Mach number as that of the original wing. No attempt was made to tune the locations of the geometry variables. For each airfoil section, camber variables were placed at every 0.2 in $x / c$, starting at 0.1 and ending at $0.9 x / c$. These camber bumps are given by quintic B-splines which interpolate the amplitude of the central knot and vanish at neighboring end-point knot locations. The defining airfoil sections were placed at $0.0,0.2,0.4,0.6,0.75,0.90$ and 1.0 in fractions of semi-span. This allows the entire wing to change shape, including both the wing root and the wing tip. To prevent a singular problem definition, the twist variable at the wing root was fixed at 0 . All variables express a delta change in geometry from the initial wing.

The optimization used the variables described above to minimize the drag as computed by the farfield formulation of induced, profile, and wave drags. This was subject to the fixed lift constraint, and a set of transpiration modeling constraints which are necessary to keep the optimization step size within the domain of applicability of the model problem which is constructed on each Tranair grid. The optimizer used was the sequential quadratic programming algorithm, NPSOL. ${ }^{37,38}$ The largest camber change was approximately $1.4 \mathrm{~mm}$ in full scale units, with the twist change being between $\pm 0.5^{\circ}$.

Additional information regarding the design of these wings is provided by Zickuhr and Tinoco ${ }^{31}$ in the workshop presentations on geometry review.

\section{Test Cases}

The success of the DPW Series is due in large part to the significant amount of personal time and computing resources invested by the participants of the workshops. In order to keep these individual investments from growing out of control, the organizing committee decided to essentially manage DPW-III as two subworkshops within one. Participants were allowed to select one of the following two cases, but were then required to provide all data mandated for that test case. Both cases are blind tests, the first on rather simple wing/body configurations, the second on extremely simple wing-alone shapes. Both cases include a single-point grid-sensitivity study, and an alpha-sweep on a medium-size grid. Note that a fixed-lift condition requires convergence on $\alpha$; this in turn adds additional effort. In addition, the CFD solutions for both test cases were required to represent fully turbulent flow as closely as possible.

In order to collect a consistent set of data from each participant, the organizing committee supplied template dataset files. These templates requested lift, drag (broken down by mechanical component), pitching moment, pressure distributions at specified span stations, trailing-edge separation locations, dimensions of the side-of-body separation bubble, grid family and sizes, turbulence model, computing platform and code performance, number of processors used, number of iterations required, etc. These workshops capture an extensive amount of information that serves as a snapshot of the industry capabilities of the time. For example, in the three workshops held thus far, one obvious trend is that the size of a typical wing/body grid has grown dramatically; the average of size of the Medium WB structured-meshes in DPW-I, DPW-II and DPW-III have been 3.2, 5.4 and 7.8 million grid points, respectively. This represents a growth rate of $30 \%$ per year between DPW-I and DPW-II, and 13\% per year between the last two workshops. Does this indicate that engineering applications are finally approaching the grid resolutions needed for accurate drag predictions on a wing/body configuration? We will at least show that the engineering-class grid resolutions (DPW Medium grids) are likely within the asymptotic range of grid convergence for many, yet not all, of the solvers represented herein.

\section{Case 1: DLR-F6 WB with and without FX2B Fairing}

The first test case is based on the DLR-F6 wing/body configuration. This case study was constructed to serve two purposes: a) provide a link to DPW-II, and $b$ ) test a hypothesis that pockets of flow separation can be a root cause for results showing poor grid convergence. 
Participants were required to provide data for both the baseline DLR-F6 geometry and one that incorporates the FX2B fairing at the following flow conditions on the specified grids.

Fixed-C $C_{L}$ Single Point Grid Sensitivity Study on Three Grids

- $M a c h=0.75, C_{L}=0.5, R e=5$ million.

Drag Polar on Medium Grid

- Mach $=0.75, R e=5$ million, $\alpha=\left[-3.0^{\circ},-2.0^{\circ},-1.0^{\circ},-0.5^{\circ}, 0.0^{\circ}, 0.5^{\circ}, 1.0^{\circ}, 1.5^{\circ}\right]$.

\section{Case 2: DPW-W1/W2 Wing-Alone}

The second test case is based on generic wing-alone geometries designed by members of the organizing committee. Participants were required to provide data for both wings at the following flow conditions on the specified grids.

Fixed- $\alpha$ Single Point Grid Sensitivity Study on Four Grids

- $M a c h=0.76, \alpha=0.5^{\circ}, R e=5$ million.

Drag Polar on Medium Grid

- $M a c h=0.76, R e=5$ million, $\alpha=\left[-1.0^{\circ}, 0.0^{\circ}, 0.5^{\circ}, 1.0^{\circ}, 1.5^{\circ}, 2.0^{\circ}, 2.5^{\circ}, 3.0^{\circ}\right]$.

\section{Baseline Grids}

An overview of the baseline grids is provided in this section. However, the details of these grids are not included herein. For more information regarding these grids, please refer to the companion papers that accompany this summary document. Tinoco ${ }^{39}$ provides descriptions of the Tinoco multiblock structured and AFLR unstructured grid families. Sclafani ${ }^{40}$ provides descriptions of the Sclafani overset structured grid families. Mavriplis ${ }^{41}$ provides descriptions of the LaRC unstructured mesh families. Brodersen ${ }^{42}$ provides descriptions of the DLR hybrid mesh families.

Because of the variation of grid types needed, a set of gridding guidelines, listed below, was established to help facilitate the creation of these grids. The gridding guidelines were provided to the persons responsible for generating the baseline grids in an attempt to maintain some level of uniformity across all types of meshes. Note that each grid family is required to include a Coarse (C), Medium (M), and Fine (F) grid; Case 2 also required an Extra-Fine (X) grid. Further, the organizing committee decided that the Medium mesh should be representative of current engineering applications of CFD being used to estimate absolute drag levels on similar configurations. For unstructured meshes, the size of the Medium mesh is also a function of the intended flow solver. For example, a cell-centered scheme has about 5.5 times the numbers of unknowns as that of a nodal scheme for a given unstructured tetrahedral mesh, with the ratio being closer to 3.5 for typical hybrid meshes. In the tables that follow, only number of grid points are given.

Table I provides the number of grid points for each grid family, configuration, and resolution for Case 1. The Tinoco through AFLR families were baseline grids provided by the organizing committee and are available to the public domain. (Although not shown in this table, the Tinoco grid family also includes a Medium-Fine mesh for both wing/body configurations.) The Embraer through TAS grids were generated by individual participants; they were not generally available before the workshop (they currently are) and are included here to document grid sizes to the extent known. The types of meshes used include multiblock (MB), overset (OS), unstructured (UN), and hybrid (HY); the LaRC grid family was used in unstructured and hybrid states (UH). These grids range in size from 2.3-to-41.1 million points. Also included in this table is a mapping of the participants who used each grid. The usage key is described in Section V.

Table II provides the number of grid points for each grid family, configuration, and resolution for Case 2. All except the JFLO grid family were provided by the organizing committee and are freely available to the public domain. The Tinoco and JFLO families are multiblock; the Sclafani grid family is overset; the DLR grid family is hybrid; the LaRC and Raytheon grid families are unstructured tetrahedral, although these meshes can be converted to hybrid. Case 2 families include an Extra-Fine mesh in the sequence. The grids range in size from 0.95 -to-55.0 million points. 
Gridding Guidelines

- Boundary Layer Region

$-Y^{+} \leq\left[1, \frac{2}{3}, \frac{4}{9}, \frac{8}{27}\right][C, M, F, X]$

$-\Delta_{1} \sim 0.0006 \mathrm{~mm}$, [Approximate dimensional spacing for $Y^{+}=1$ ]

$-\Delta_{2}=\Delta_{1}$, [Two cell layers of constant spacing at viscous wall]

- Growth Rates $\leq 1.25$, [Preferably $\leq 1.20]$

- Farfield: $\sim 100 C_{r e f}$-lengths away from geometry

- Local Spacings on Medium Grid

- Chordwise: $0.1 \%$ local chord at Wing Leading Edge \& Trailing Edge

- Spanwise: $0.1 \%$ semispan at root \& tip

- Cell Size on Fuselage Nose \& Tail: $2 \% C_{r e f}$

- Cells across Wing Trailing-Edge Base: $[8,12,16,24][C, M, F, X]$

- Grid Family

- Medium Mesh Representative of Current Engineering Drag Predictions

- Maintain a Parametric Family of Uniformly-Refined Grids in Sequence

- Grid Size to Grow 3X for Each Level Refinement [Structured: 1.5X in Each I,J,K Direction]

- Give Consideration to Multigridable Dimensions on Structured Meshes

- Sample Size for DLR-F6 Wing/Body: [2.7M,8M,24M] [C, $M, F]$

Table I: Case 1 DLR-F6/FX2B Grids - Number of Grid Points.

\begin{tabular}{|c|c|c|c|c|c|c|c|c|}
\hline & & & DLR-F6 & & & FX2B & & \\
\hline Family & Type & Fine & Medium & Coarse & Fine & Medium & Coarse & Usage \\
\hline Tinoco & $\mathrm{MB}$ & $27,185,664$ & $8,080,896$ & $2,298,880$ & $27,185,664$ & $8,080,896$ & $2,298,880$ & A-C,G-J \\
\hline SAUNA & $\mathrm{MB}$ & $9,761,201$ & $4,731,073$ & $2,551,989$ & $9,761,201$ & $4,731,073$ & $2,551,989$ & $\bar{D}$ \\
\hline Extruded & $\mathrm{MB}$ & $28,367,120$ & $9,343,009$ & $2,996,626$ & $28,367,120$ & $9,329,185$ & $3,028,420$ & $\mathrm{E}$ \\
\hline Gridgen & $\mathrm{MB}$ & $27,982,776$ & $8,927,196$ & $2,739,621$ & $27,982,776$ & $9,138,772$ & $2,842,878$ & $\mathrm{~F}, \mathrm{~T}$ \\
\hline Sclafani & OS & $26,892,352$ & $7,985,236$ & $2,387,918$ & $26,969,192$ & $8,020,348$ & $2,395,170$ & $\mathrm{~K}-\mathrm{M}, \mathrm{U}$ \\
\hline DLR & $\mathrm{HY}$ & $8,535,263$ & $5,102,446$ & $2,464,385$ & $10,305,876$ & $6,111,664$ & $2,873,102$ & $\mathrm{~N}$ \\
\hline ANSYS & $\mathrm{HY}$ & $18,120,772$ & $8,038,922$ & $3,059,189$ & $20,472,520$ & $8,272,308$ & $3,163,605$ & $\mathrm{O}$ \\
\hline LaRC & $\mathrm{UH}$ & $40,014,934$ & $14,298,135$ & $5,354,214$ & $41,069,036$ & $14,598,610$ & $5,618,073$ & $\mathrm{P}, \mathrm{Q}$ \\
\hline AFLR & UN & $11,374,451$ & $3,792,485$ & $1,492,082$ & $11,849,212$ & $3,178,559$ & $1,640,590$ & $\mathrm{R}, \mathrm{S}, \mathrm{Y}$ \\
\hline Embraer & $\mathrm{UN}$ & $24,030,000$ & $8,320,000$ & $3,550,000$ & $24,030,000$ & $8,320,000$ & $3,550,000$ & $\mathrm{~V}$ \\
\hline STAR & UN & - & $12,377,058$ & - & $21,509,137$ & $12,469,599$ & $8,421,799$ & $\mathrm{~W}$ \\
\hline USM3D & $\mathrm{UN}$ & - & - & - & - & - & - & $\mathrm{X}$ \\
\hline TAS & $\mathrm{UN}$ & $17,535,215$ & $9,431,154$ & $5,399,929$ & $17,219,535$ & $9,481,477$ & $5,422,128$ & $\mathrm{Z}$ \\
\hline
\end{tabular}

Table II: Case 2 DPW-W1/W2 Grids - Number of Grid Points.

\begin{tabular}{|c||c|c|c|c||c|c|c|c|}
\hline \multicolumn{1}{|c||}{} & \multicolumn{5}{c||}{ DPW-W1 } & \multicolumn{4}{c|}{ DPW-W2 } \\
\hline Family & Extra-Fine & Fine & Medium & Coarse & Extra-Fine & Fine & Medium & Coarse \\
\hline \hline Tinoco & $14,811,489$ & $8,620,123$ & $4,204,203$ & $1,602,651$ & $14,811,489$ & $8,620,123$ & $4,204,203$ & $1,602,651$ \\
\hline Sclafani & $55,014,321$ & $16,265,909$ & $4,856,149$ & $1,442,285$ & $55,014,321$ & $16,265,909$ & $4,856,149$ & $1,442,285$ \\
\hline DLR & $17,053,510$ & $10,150,588$ & $5,288,507$ & $2,174,364$ & $16,631,805$ & $9,910,645$ & $5,030,379$ & $1,928,405$ \\
\hline LaRC & $36,956,019$ & $11,492,625$ & $4,495,117$ & $1,818,508$ & $38,462,630$ & $11,903,329$ & $4,658,853$ & $1,882,672$ \\
\hline Raytheon & $12,748,678$ & $6,138,245$ & $2,417,082$ & 983,633 & $12,419,567$ & $5,963,713$ & $2,325,884$ & 947,409 \\
\hline JFLO & - & - & - & - & - & - & - & - \\
\hline
\end{tabular}




\section{Results}

Participants of the DPW-III were required to provide results on either the wing/body or wing-alone test case. To their credit, many participants chose to investigate both cases; several provided multiple datasets on a given case. This section summarizes the data collected on these test cases. These data are then used to estimate absolute drag levels for the DLR-F6 with and without the FX2B fairing, and lift-to-drag ratios of the DPW-W1 and DPW-W2 wings. These estimates are made prior to any experimental data being available (at test-case Reynolds number) on any of the configurations. There were twenty-six submissions for Case 1 and eleven for Case 2. To help minimize confusion, the Case 1 submissions are tagged with a single capitalized letter from A-Z, while the Case 2 submissions are labeled 1-11. The following subsections detail the results for the two cases.

\section{Case 1: DLR-F6 WB with and without FX2B Fairing}

The first test case is the DLR-F6 wing/body configuration with and without the FX2B fairing. Table III provides the CFD code, grid type, grid family, turbulence model, and submitter name and organization for each block of data submitted. Twenty of the twenty-six submissions were complete in the sense that they included all of the mandatory data requested. While almost all of the data were supplied by the six remaining, these data blocks could not be used to conduct a consistent Richardson extrapolation to the continuum in the grid-sensitivity study.

Table III: Case 1 Submissions.

\begin{tabular}{|c|c|c|c|c|c|}
\hline Tag & Code & Grid Type & Grid Family & Turbulence Model & Submitter \\
\hline \hline A & PAB3D & Multiblock & Tinoco & Girimaji EASM & ASM Elmiligui \\
\hline B & PAB3D & Multiblock & Tinoco & K-Epsilon & ASM Elmiligui \\
\hline C & PAB3D & Multiblock & Tinoco & SZL EASM & ASM Elmiligui \\
\hline D & STAR-CCM+ & Multiblock & SAUNA & Wilcox K-Omega & QinetiQ Milne \\
\hline E & UPACS & Multiblock & Extruded & Modified SA & JAXA Murayama \\
\hline F & UPACS & Multiblock & Gridgen & Modified SA & JAXA Murayama \\
\hline G & CFL3D-Thin & Multiblock & Tinoco & SA & Boeing Tinoco \\
\hline H & CFL3D-Thin & Multiblock & Tinoco & SST & Boeing Tinoco \\
\hline I & CFL3D-Full & Multiblock & Tinoco & SA & Boeing Tinoco \\
\hline J & CFL3D-Full & Multiblock & Tinoco & SST & Boeing Tinoco \\
\hline K & CFL3D-Full & Overset & Sclafani & SST & LaRC Rumsey \\
\hline L & CFL3D-Thin & Overset & Sclafani & SST & LaRC Rumsey \\
\hline M & Overflow & Overset & Sclafani & SA & Boeing Sclafani \\
\hline N & TAU & Hybrid & DLR & SA Edwards & DLR Brodersen \\
\hline O & Edge & Hybrid & ANSYS CFX & Hellsten EARSM & FOI Eliason \\
\hline P & FUN3D & Unstructured & LaRC Nodal & SA & LaRC Lee-Rausch \\
\hline Q & NSU3D & Hybrid & LaRC Mixed & SA & UWy Mavriplis \\
\hline R & CFD++ & Hybrid & AFLR & SA & Boeing Venkat \\
\hline S & BCFD & Hybrid & AFLR & SA & Boeing Winkler \\
\hline T & UPACS & Multiblock & Gridgen & SST & JAXA Murayama \\
\hline U & OVERFLOW & Overset & Sclafani & SST & LaRC Rumsey \\
\hline V & FLUENT & Unstructured & Embraer & K-Epsilon & Fluent Scheidigger \\
\hline W & STAR-CCM+ & Unstructured & STAR & SST & CD-Adapco Vaughn \\
\hline X & USM3D & Unstructured & USM3D & SA/WF & Raytheon Venkat \\
\hline Y & BCFD & Hybrid & AFLR & SST & Boeing Winkler \\
\hline Z & TAS & Unstructured & TAS & Modified SA & JAXA Murayama \\
\hline
\end{tabular}


A representative example of the wing pressure distributions at the design condition of $M=0.75, C_{L}=0.5$, and $R e=5$ million is given in Figure 2. In this example, the $15 \%$ semispan (near side-of-body) uppersurface pressure distribution of the baseline DLR-F6 clearly exhibits signs of flow separation, while the flow about the FX2B geometry is well attached. In general, the DPW-III submissions unanimously agreed that the flow was well attached for the FX2B configuration, however, they were divided dramatically on the size of the side-of-body separation bubble for the baseline DLR-F6. More discussion on this will follow.

\section{Case 1a: Fixed- $C_{L}$ Single-Point Grid Sensitivity Study}

Figures 3-6 provide grid-sensitivity trends for idealized profile drag at the single-point fixed- $C_{L}$ condition. In these figures, side-by-side comparison plots are shown, where the baseline DLR-F6 data is captured in the left plot and the FX2B data on the right. The curves of these plots are labeled with the tag letter given in Table III. Note that all data blocks based on the Tinoco grid family also include results from a Medium-Fine grid; for consistency, these data are not included in the tables, nor were they utilized in the ranking procedure described at the end of this subsection. Figure 3 includes all data from all submissions. At first glance of this overview plot, the scatter in this data can be quite disheartening. Recall that the Medium mesh is supposed to represent the current engineering practice. In these figures, the Medium mesh grid factor falls within the range [2E-05, 3E-05]. Hence, the range of the idealized profile drag scatter of the current engineering practice is on the order of 50 counts, for either configuration. A companion paper by Morrison and Hemsch ${ }^{43}$ will address this scatter and better quantify confidence levels through a rigorous statistical analysis of the data.

The composite curves of Figure 3 are broken out by grid type in Figures 4-6. Figure 4 illustrates the trend of the multiblock CFD results. In this figure, note that three different multiblock families are shown. The solid lines represent results using the Tinoco grid family; the dashed lines capture the Gridgen families; the chain-dot refer to the SAUNA grid family. For the DLR-F6 baseline, note that if extrapolated to the continuum, many of the Tinoco-grid-family results indicate a low-drag value, while the Gridgen-grid-family data point to a higher value. For the FX2B geometry, the data associated with these two grid families are more comparable. Figure 5 shows the grid-sensitivity trends for the Sclafani-grid-family of overset-grid results. Figure 6 provides the corresponding trends of the unstructured and hybrid CFD solutions. In this figure, note that the unstructured-mesh families are grouped. The solid lines represent results based on the LaRC grid families; the dashed lines depict results using the AFLR grid families; the chain-dot lines capture data based on the five remaining unstructured-mesh families.

Figure 7 illustrates the size and location of the side-of-body separation bubble for the baseline DLR-F6 wing/body configuration, as computed by the participants. The air flow is from left to right, the bold vertical line depicts the wing trailing edge, while the bold curved horizontal line is the wing/body intersection. The A-Z symbols plotted in this figure represent the (X-EYE,Y-EDGE) coordinates of the computed separation bubbles. Here, X-EYE depicts the streamwise position of the bubble eye (which is approximately the streamwise location of the maximum bubble width), and Y-EDGE is the maximum spanwise extent of the bubble. For reference, these data are overlayed on an oil-flow pattern taken from an ONERA wind-tunnel test. However, note that the oil-flow pattern of the test corresponds to a lower Reynolds-number flow. For clarity, these data are organized into three subplots by grid type. This figure illustrates that there is large scatter in the computed sizes of the separation bubble, represented by Y-EDGE, even within the common grid types.

Upon reviewing the previous five figures, it appears visually that some of the grid-convergence data may be classified as outliers. How to quantify a block of data as an outlier can be a rather tricky matter. Nonetheless, there is an enormous amount of information encoded in the database provided by the participants and presented previously in Figures 3-6. It would seem a shame not to try to decypher this data in an attempt to extract drag values. At the risk of failure, the first author includes herein an approach to identify outliers. The basic idea is to develop a measure-of-merit for each block of CFD data, rank the data blocks by this merit, and then cull out the solutions that represent outliers.

If a Richardson extrapolation is performed on a pair of data from a grid-sensitivity curve, an estimate of the continuum value is obtained. If this extrapolation is performed using data from the Fine and Medium meshes, the resulting value does not necessarily equal that of a similar extrapolation using data from the Medium and Coarse meshes. If the two values are equal, the trend line is exactly straight, and thus meets the necessary (but insufficient) requirement that data from all three meshes fall within an asymptotic range. The difference of these two extrapolated values provides a quantifiable measure of how the data deviates from a

Vassberg, et al., AIAA Paper 2007-0260, Reno, NV, January 2007 
straight line. For the constant-lift grid-sensitivity of Case 1, this measure-of-merit could be comprised of $\alpha$, $C_{D}, C_{D p r}, C_{D s f}$, or $C_{M}$. In the following Lagrange equation, let $\mathcal{Y}$ represent any one of these quantities, and $\mathcal{X}=N^{-\frac{2}{3}}$. Here, $N$ is the total number of grid points in the grid system. As defined, $\mathcal{X}$ is an appropriate parameter for a second-order scheme applied to results on a parametric family of three-dimensional meshes that have been uniformly refined in all three coordinate directions.

$$
\begin{gathered}
\mathcal{Y}_{F M}=\frac{\mathcal{X}_{M} \mathcal{Y}_{F}-\mathcal{X}_{F} \mathcal{Y}_{M}}{\mathcal{X}_{M}-\mathcal{X}_{F}}, \quad \mathcal{Y}_{M C}=\frac{\mathcal{X}_{C} \mathcal{Y}_{M}-\mathcal{X}_{M} \mathcal{Y}_{C}}{\mathcal{X}_{C}-\mathcal{X}_{M}} \\
\mathcal{D}(\mathcal{Y})=\left|\mathcal{Y}_{F M}-\mathcal{Y}_{M C}\right|
\end{gathered}
$$

Here, $\mathcal{Y}_{F M}$ and $\mathcal{Y}_{M C}$ represent the continuum estimates using the Fine/Medium and Medium/Coarse pairs of data, respectively, and $\mathcal{D}(\mathcal{Y})$ is the absolute value of their difference.

Unfortunately, the level to which the uniform-refinement critereon has been achieved varies across the set of baseline grid families. For structured meshes, this requirement can be easily met by uniformly scaling the I, J \& K dimensions. For unstructured meshes, the situation is more complicated. One approach is to use a global scaling parameter related to cell sizes, but this technique is difficult to enforce uniformily and does not guarantee the mesh connectivity to be self-similar between coarse and fine meshes. Another approach is to subdivide each element into smaller elements, but this can lead to undesireable cell aspect ratios, especially in the finest grid.

Since this is the drag prediction workshop, let's build the metric based on drag. However, to avoid compensating deviations of drag parts benefiting one set of data over another, we independently determine the differences of continuum estimates of $C_{D p r}$ and $C_{D s f}$, and then sum their absolute values.

$$
\mathcal{M O M} \equiv \mathcal{D}\left(C_{D p r}\right)+\mathcal{D}\left(C_{D s f}\right)
$$

Here, $\mathcal{M O M}$ is the foundation measure-of-merit used herein to rank the blocks of data collected; the smaller the value of $\mathcal{M O M}$, the higher the quality of the data block. To further minimize the chance of other compensating errors favoring one data block over another, use the average of the DLR-F6 and FX2B values. Serendipitously, this ranks the DLR-F6 and FX2B data blocks in the same order, simplifying matters.

$$
\operatorname{Avg} \mathcal{M O M} \equiv \frac{1}{2}\left(\mathcal{M O M}_{F 6}+\mathcal{M O} \mathcal{M}_{F X 2 B}\right)
$$

A few items to note about this choice of measure-of-merit. Firstly, it is not biased by the absolute value of drag. Hence, the "correct" value of drag does not need to be known or inferred in any manner. Secondly, the measure-of-merit is not biased by the slope of the asymptotic grid convergence. It is only a function of the linearity of the grid-convergence trend lines. However, what remains somewhat ad hoc is the method to determine what value of $\mathrm{Avg} \mathcal{M O M}$ separates the outliers from the core solution sets.

As mentioned earlier, only twenty of the submissions provided sufficient data to perform a consistent set of Richarson extrapolations. These data are summarized in Table IV for the baseline DLR-F6 and in Table V for the FX2B configuration. (Recall that these tables omit all Medium-Fine data based on the Tinoco grids so that comparison across data blocks is more consistent.) The estimated continuum values in these tables are based on Fine/Medium mesh extrapolations. Of these twenty blocks of data, one did not provide the pressure and skin-friction parts of drag, and therefore cannot be ranked with the aforementioned technique. Table VI puts in rank order the remaining nineteen data blocks, and Figure 8 illustrates $\mathcal{M O} \mathcal{M}$ as ordered by averaged values. This plot is scaled to 20 counts; some data falls out of view. It is interesting that the first eight data blocks have similar DLR-F6 and FX2B $\mathcal{M O M}$ values. An unusual data block is that of Rank 12 which has a very good FX2B $\mathcal{M O M}$, yet a reasonably poor DLR-F6 value. A trend seen in this figure is that there are two noticable departures of $\mathcal{M O M}$ values, one at Rank 09, and another at Rank 14. However, the departures of Ranks 09-13 are small by comparison with those of Ranks 14-19. Based soley on this observation, Ranks 14-19 are considered outliers. 
Table IV: Case 1 DLR-F6 Data Extrapolated To Continuum.

\begin{tabular}{|c|c|c|c|c|c|c|}
\hline Tag & $\alpha$ & $C_{D}$ & $C_{D . P R}$ & $C_{D . S F}$ & $C_{M}$ & $C_{D}-C_{L}^{2} /(\pi A R)$ \\
\hline \hline A & 0.0244 & 0.02686 & 0.01404 & 0.01283 & -0.14617 & 0.01843 \\
\hline B & -0.0614 & 0.02846 & 0.01420 & 0.01425 & -0.15065 & 0.02005 \\
\hline C & 0.1518 & 0.02613 & 0.01428 & 0.01185 & -0.13747 & 0.01778 \\
\hline D & -0.0990 & 0.02732 & 0.01470 & 0.01261 & -0.15342 & 0.01894 \\
\hline E & 0.1824 & 0.02761 & 0.01552 & 0.01209 & -0.14624 & 0.01930 \\
\hline F & 0.2003 & 0.02772 & 0.01564 & 0.01208 & -0.14602 & 0.01941 \\
\hline G & -0.1633 & 0.02622 & 0.01392 & 0.01231 & -0.15753 & 0.01784 \\
\hline H & 0.0472 & 0.02641 & 0.01438 & 0.01203 & -0.14280 & 0.01803 \\
\hline I & -0.0909 & 0.02636 & 0.01402 & 0.01235 & -0.14770 & 0.01799 \\
\hline J & 0.0863 & 0.02658 & 0.01453 & 0.01205 & -0.13211 & 0.01820 \\
\hline K & 0.2692 & 0.02747 & 0.01544 & 0.01205 & -0.13538 & 0.01910 \\
\hline L & 0.0737 & 0.02695 & 0.01485 & 0.01210 & -0.14450 & 0.01857 \\
\hline M & 0.2663 & 0.02759 & 0.01561 & 0.01199 & -0.13944 & 0.01923 \\
\hline N & 0.1022 & 0.02612 & 0.01335 & 0.01252 & -0.15245 & 0.01775 \\
\hline O & 0.0400 & 0.02710 & 0.01375 & 0.01334 & -0.16255 & 0.01844 \\
\hline P & 1.2476 & 0.03226 & 0.02193 & 0.01035 & -0.11064 & 0.02396 \\
\hline Q & 0.1006 & 0.02626 & 0.01473 & 0.01151 & -0.14349 & 0.01788 \\
\hline R & -1.1783 & 0.03325 & - & - & - & 0.02487 \\
\hline S & 0.4938 & 0.02939 & 0.01740 & 0.01199 & -0.12926 & 0.02101 \\
\hline Z & -0.1089 & 0.02846 & 0.01628 & 0.01215 & -0.15433 & 0.02002 \\
\hline
\end{tabular}

Table V: Case 1 FX2B Data Extrapolated To Continuum.

\begin{tabular}{|c|c|c|c|c|c|c|}
\hline Tag & $\alpha$ & $C_{D}$ & $C_{D . P R}$ & $C_{D . S F}$ & $C_{M}$ & $C_{D}-C_{L}^{2} /(\pi A R)$ \\
\hline \hline $\mathrm{A}$ & 0.1296 & 0.02701 & 0.01409 & 0.01293 & -0.13977 & 0.01860 \\
\hline B & 0.0950 & 0.02854 & 0.01430 & 0.01422 & -0.14129 & 0.02013 \\
\hline C & 0.2210 & 0.02639 & 0.01438 & 0.01199 & -0.13328 & 0.01798 \\
\hline D & -0.1150 & 0.02705 & 0.01427 & 0.01275 & -0.15310 & 0.01867 \\
\hline E & -0.0887 & 0.02628 & 0.01393 & 0.01235 & -0.15479 & 0.01790 \\
\hline F & -0.0888 & 0.02628 & 0.01391 & 0.01237 & -0.15489 & 0.01790 \\
\hline G & -0.0569 & 0.02624 & 0.01382 & 0.01244 & -0.15189 & 0.01786 \\
\hline H & 0.1178 & 0.02639 & 0.01423 & 0.01217 & -0.13818 & 0.01802 \\
\hline I & -0.0673 & 0.02636 & 0.01388 & 0.01251 & -0.14343 & 0.01798 \\
\hline J & 0.1180 & 0.02655 & 0.01437 & 0.01220 & -0.13562 & 0.01818 \\
\hline K & 0.0507 & 0.02656 & 0.01438 & 0.01220 & -0.14227 & 0.01818 \\
\hline L & 0.0493 & 0.02643 & 0.01425 & 0.01220 & -0.14353 & 0.01805 \\
\hline M & -0.0907 & 0.02596 & 0.01366 & 0.01230 & -0.14997 & 0.01759 \\
\hline N & -0.1414 & 0.02527 & 0.01324 & 0.01202 & -0.16042 & 0.01691 \\
\hline O & 0.1570 & 0.02727 & 0.01472 & 0.01255 & -0.13316 & 0.01875 \\
\hline P & 0.3866 & 0.02584 & 0.01505 & 0.01077 & -0.11826 & 0.01746 \\
\hline Q & 0.2634 & 0.02606 & 0.01444 & 0.01161 & -0.13338 & 0.01768 \\
\hline R & -0.0405 & 0.02934 & - & - & - & 0.02097 \\
\hline S & 0.1207 & 0.02795 & 0.01569 & 0.01226 & -0.13778 & 0.01957 \\
\hline Z & -0.1140 & 0.02627 & 0.01397 & 0.01228 & -0.15451 & 0.01789 \\
\hline
\end{tabular}


Figure 9 provides the ranked continuum estimates of total drag for both DLR-F6 and FX2B configurations. Applying a simple statistical reduction of this data, we have:

$$
\begin{aligned}
F 6: \quad \text { Avg. } C_{D} & =0.02690 \\
\sigma & =0.00060 \\
\min & =0.02612 \\
\max & =0.02772
\end{aligned}
$$

$$
\begin{aligned}
F X 2 B: \quad \text { Avg. } C_{D} & =0.02636 \\
\sigma & =0.00049 \\
\min & =0.02527 \\
\max & =0.02727 .
\end{aligned}
$$

Here, $\sigma$ is the standard deviation of the data.

Table VI: Case 1 Continuum Drag Estimates Ranked By Avg $\mathcal{M O M}$.

\begin{tabular}{|c|c|c|c|c|c|c|}
\hline Rank & AvgMoM & F6-Merit & FX2B-Merit & Tag & F6. $C_{D}$ & FX2B. $C_{D}$ \\
\hline \hline 1 & 0.00010 & 0.00007 & 0.00013 & $\mathrm{I}$ & 0.02636 & 0.02636 \\
\hline 2 & 0.00012 & 0.00007 & 0.00017 & $\mathrm{G}$ & 0.02622 & 0.02624 \\
\hline 3 & 0.00013 & 0.00012 & 0.00014 & $\mathrm{~F}$ & 0.02772 & 0.02628 \\
\hline 4 & 0.00021 & 0.00021 & 0.00022 & $\mathrm{E}$ & 0.02761 & 0.02628 \\
\hline 5 & 0.00022 & 0.00015 & 0.00029 & $\mathrm{H}$ & 0.02641 & 0.02639 \\
\hline 6 & 0.00024 & 0.00025 & 0.00023 & $\mathrm{M}$ & 0.02759 & 0.02596 \\
\hline 7 & 0.00026 & 0.00023 & 0.00029 & $\mathrm{~J}$ & 0.02658 & 0.02655 \\
\hline 8 & 0.00038 & 0.00039 & 0.00037 & $\mathrm{Q}$ & 0.02626 & 0.02606 \\
\hline 9 & 0.00063 & 0.00089 & 0.00037 & $\mathrm{~N}$ & 0.02612 & 0.02527 \\
\hline 10 & 0.00083 & 0.00103 & 0.00062 & $\mathrm{~L}$ & 0.02695 & 0.02643 \\
\hline 11 & 0.00094 & 0.00122 & 0.00067 & $\mathrm{~K}$ & 0.02747 & 0.02656 \\
\hline 12 & 0.00096 & 0.00180 & 0.00012 & $\mathrm{O}$ & 0.02710 & 0.02727 \\
\hline 13 & 0.00100 & 0.00088 & 0.00112 & $\mathrm{D}$ & 0.02732 & 0.02705 \\
\hline 14 & 0.00125 & 0.00192 & 0.00059 & $\mathrm{Z}$ & 0.02846 & 0.02627 \\
\hline 15 & 0.00157 & 0.00224 & 0.00090 & $\mathrm{P}$ & 0.03226 & 0.02584 \\
\hline 16 & 0.00244 & 0.00413 & 0.00075 & $\mathrm{~S}$ & 0.02939 & 0.02795 \\
\hline 17 & 0.00327 & 0.00269 & 0.00386 & $\mathrm{C}$ & 0.02613 & 0.02639 \\
\hline 18 & 0.00336 & 0.00280 & 0.00391 & $\mathrm{~A}$ & 0.02686 & 0.02701 \\
\hline 19 & 0.00715 & 0.00662 & 0.00769 & $\mathrm{~B}$ & 0.02846 & 0.02854 \\
\hline
\end{tabular}

Recall that this discussion began with a disheartening statement regarding the large spread of data shown in Figure 3. If there is any merit to the above filtering of outliers, then the situation of data scatter looks much better, albeit not at the Medium-mesh level, but rather with the continuum estimates. Now the standard deviation of absolute drag levels is about $2 \%$. Furthermore, the scatter of the data of the DLRF6 results, as quantified by $\sigma$, is statistically equivalent to that of the FX2B. This, in and of itself, is an interesting observation. Does this contradict our hypothesis that pockets of flow separation are a root cause of grid-convergence issues? Not necessarily. This may be partially a consequence of the measure-of-merit chosen, and how the metric was used to identify outliers. As noted before, Ranks 01-08 have very similar values of $\mathcal{M O M}$ for the DLR-F6 and FX2B configurations. This indicates that (for these data blocks) grid convergence is not being adversely affected by the pockets of flow separation. However, in all but one of Ranks 09-16, the FX2B $\mathcal{M O M}$ is significantly better than that of DLR-F6. These data blocks appear to uphold our hypothesis. The values of $\mathcal{M O M}$ for Ranks 17-19 are significantly bad for either configuration and are not used to draw a conclusion on this point. So it seems that pockets of flow separation can aggravate grid convergence, but not necessarily. This is somewhat of an unexpected finding of the DPW-III.

Reconsider Figure 9, but now focus on the DLR-F6 data, open-square symbols. Notice that there appears to be two distinct levels of drag, one in the band of 261-to-266 counts, and the other in the 270-to-277 range, separated by a 4 -count void. Is a 4 -count void that separates a 5 -count cluster and a 7 -count group 
statistically significant? Probably not with only 13 data samples, but let's further investigate these groupings anyway. The first band is comprised of 6 data from 3 participants using 3 grid families; the second band is comprised of 7 data from 5 participants using 5 grid families. Hence, the computed double-valued levels of drag do not appear to be explained by any single main effect that we have considered (e.g., grid type, CFD user, or even by turbulence model). It could be that we have not identified the correct effect, or it could be that it is a more complex interaction between main effects; at this time, we do not know. Applying a statistical reduction of the two bands of DLR-F6 data gives:

$\begin{aligned} F 6_{1}: \quad A v g \cdot C_{D 1} & =0.02632, & F 6_{2}: \quad A v g . C_{D 2} & =0.02739, \\ \sigma_{1} & =0.00016, & \sigma_{2} & =0.00029, \\ \min _{1} & =0.02612, & \min _{2} & =0.02695, \\ \max _{1} & =0.02658, & \max _{2} & =0.02772 .\end{aligned}$

From these statistical reductions, we have three guesses at the delta-drag between the DLR-F6 baseline and the FX2B configurations. However, due to the small number of samples, we note that the statistical values given by $\Delta C_{D 1}$ and $\Delta C_{D 2}$ may not be significantly different from that of $\Delta C_{D 0}$.

$$
\begin{aligned}
(F X 2 B-F 6): \quad \Delta C_{D 0} & =-0.00054, \\
\Delta C_{D 1} & =+0.00004, \\
\Delta C_{D 2} & =-0.00103 .
\end{aligned}
$$

Figure 10 provides the rank-ordered estimates of $\Delta C_{D}$ from each data block. Notice that the three statistical estimates of $\Delta C_{D 012}$ appear to be present in this figure. Here, six data indicate essentially no change in drag, two have a 3-to-5-count improvement, and five data show a benefit of 8-to-17 counts.

Before leaving the discussion related to the continuum estimates, a couple of observations will be noted; there are a few back-to-back comparisons in the data where only one variable changed. For example, the drag predicted using the full Navier-Stokes equations appears to be higher than that obtained using the thin-layer approximations. The back-to-back comparisons yielding this observation come from the data block pairs of $(\mathrm{G}, \mathrm{I}),(\mathrm{H}, \mathrm{J})$, and $(\mathrm{L}, \mathrm{K})$ for both the DLR-F6 and FX2B results; all six pairs exhibit this trend.

Another comparison that can be made is regarding the SA and SST turbulence models. Here refer to the data block pairs of $(\mathrm{G}, \mathrm{H}),(\mathrm{I}, \mathrm{J})$, and $(\mathrm{M}, \mathrm{U})$. Although we do not have sufficient data for a continuum estimate of the U data block, we can compare the DLR-F6 Medium grid results with those of the M data block. In this comparison, all five pairs of data show that the total drag from the SST model is higher than that from the SA model. Digging further into this SA/SST comparison shows that the pressure drag of the SST model is always higher than that of the SA model, yet the skin-friction drag shows the opposite trend in all cases.

In addition to the above trends identified, other trends are currently being investigated by participants of the DPW-III with post-workshop studies. As an example, Sclafani ${ }^{40}$ has studied the effect of an extreme refinement of the grid near the wing trailing edge. Also, he has added an Extra-Fine mesh to his overset grid families; the results on the baseline DLR-F6 show that the side-of-body bubble monotonically grows with increasing grid refinement. Further, the size of the bubble is consistently larger when the flow is computed with the full Navier-Stokes equations as compared with that of the thin-layer approximations.

\section{Case 1b: Drag Polar on Medium Grid Study}

The remainder of this subsection provides the alpha-sweep data. For the sake of clarity, these data are presented by grid type using similar side-by-side plots as before, with DLR-F6 on the left and FX2B on the right.

Figures 11-12 provide the idealized profile drag and skin-friction drag, respectively, of the data blocks based on multiblock structured grids. Note that these curves have been further itemized by grid family sets. The solid lines represent results using the Tinoco grid families; the dashed lines capture the Gridgen families; the chain-dot refer to the SAUNA grid families. In these figures, data block B appears to be an outlier related to the idealized profile drag, and data block A looks like an outlier with respect to skin friction. Both of these data blocks were identified as outliers by the filtering process of the grid-sensitivity study above. In 
addition to these, the $\mathrm{C}$ data block was also identified as an outlier; based on these figures, the $\mathrm{C}$ data block appears to be on the fringe in the polars of both drag components.

Figures 13-14 provide drag-polar results based on the overset mesh family. Note that the shapes of the idealized profile drag polars for the $\mathrm{K}$ data block are unlike those of any other data block. Yet, this data block was not captured as an outlier by the filter. Upon further inspection, it was noticed that the grid-sensitivity data from the $\mathrm{K}$ submission were not compatible with its own polars. The cause of this inconsistency was tracked down to different processes being used to compute the fixed- $C_{L}$ results from those of the $\alpha$-sweep. As a consequence, these data are updated in a companion paper ${ }^{40}$ focused on overset-mesh drag predictions. The filter for outliers was not designed to capture this type of inconsistency.

Figures 15-16 address the unstructured and hybrid mesh data. These data are also grouped. The solid lines represent results based on the LaRC grid families; the dashed lines depict results using the AFLR grid families; the chain-dot lines capture data based on the five remaining unstructured-mesh families. Here, the $\mathrm{S}$ and $\mathrm{P}$ data blocks appear to be outliers on the DLR-F6 idealized profile drag polars; the $\mathrm{S}$ block is also an extreme on the FX2B scatter. Refering to the skin-friction polars, the P and W data blocks look like they could also be outliers. The filter identified P and S as outliers; the W data block had insufficient grid-convergence data to be analyzed.

For completeness, lift and pitching moment curves are included next. Figure 17 shows the lift curves. Here, note that the $\mathrm{P}$ data block appears to be an outlier on the DLR-F6 lift curve. Figure 18 shows the pitching moment curves. Although none of the pitching-moment curves stand out as clearly being outliers, it is noted that the $\mathrm{P}$ and $\mathrm{S}$ data blocks fall on the extreme low side of pitching-moment values.

\section{Case 2: DPW-W1/W2 Wing-Alone}

The second test case is based on the wing-alone geometries developed by the organizing committee. Table VII provides the CFD code, grid type, grid family, turbulence model, and submitter name and organization for each block of data submitted. Ten of the eleven submissions were complete in the sense that they included all of the mandatory data requested. These data were used to conduct a consistent Richardson extrapolation to the continuum in the grid-sensitivity study that follows.

Table VII: Case 2 Submissions.

\begin{tabular}{|c|c|c|c|c|c|}
\hline Tag & Code & Grid Type & Grid Family & Turbulence Model & Submitter \\
\hline \hline 1 & FLUENT & Multiblock & Tinoco & K-Epsilon & Fluent Scheidigger \\
\hline 2 & CFL3D-Thin & Multiblock & Tinoco & SA & Boeing Tinoco \\
\hline 3 & CFL3D-Thin & Multiblock & Tinoco & SST & Boeing Tinoco \\
\hline 4 & OVERFLOW & Overset & Sclafani & SA & Boeing Sclafani \\
\hline 5 & TAU & Hybrid & DLR & SA Edwards & DLR Brodersen \\
\hline 6 & FUN3D & Unstructured & LaRC Nodal & SA & LaRC Lee-Rausch \\
\hline 7 & NSU3D & Hybrid & LaRC Mixed & SA & UWy Mavriplis \\
\hline 8 & NSU3D & Unstructured & Raytheon & SA & Cessna Zickuhr \\
\hline 9 & FLOWer & Multiblock & Tinoco & RSM & DLR Eisfeld \\
\hline 10 & FLOWer & Multiblock & Tinoco & SST & DLR Raddatz \\
\hline 11 & TAI & Multiblock & JFLO C-H & BL & TAI Tarhan \\
\hline
\end{tabular}

A representative example of the DPW-W1 wing pressures and upper-surface streamlines at the design condition of $M=0.76, \alpha=0.5^{\circ}$, and $R e=5$ million is given in Figures 19-20. Figure 20 overplots the $55 \%$ semispan pressure distributions as computed on the Coarse, Medium, Fine, and Extra-Fine meshes. Notice that there is an almost imperceptible difference of these pressures between all but the Coarse mesh solution. This behavior is typical of all of the data submitted on this case to the DPW-III.

\section{Case 2a: Fixed- $\alpha$ Single-Point Grid Sensitivity Study}

Figures 21-26 provide grid-sensitivity trends for the single-point constant-alpha condition of $M=0.76$, $\alpha=0.5^{\circ}$, and $R e=5$ million. These figures are organized such that the W1 data resides at the top of 
the page and the W2 data at the bottom. All curves are labeled with a number 1-9,0. Recall that this grid-sensitivity study includes four meshes.

Figures 21-22 illustrate the grid convergence of total drag. The scatter of these curves is on the order of $10 \%$, and is fairly constant with grid resolution. Figures 23-24 depict the trends of lift with respect to grid resolution. The scatter of these curves is also on the order of $10 \%$. However, since drag is a strong function of lift, and lift varies between data blocks, maybe the scatter on predicted aerodynamic performance is not as large as it seems in Figures 21-22. In an attempt to compensate for drag variation due to lift, the next two metrics are included. Figures 25-26 illustrate the idealized profile drag polars. Notice that the scatter band of this metric has been reduced to about $5 \%$ of absolute drag. Figures 27-28 depict the lift-to-drag ratio of these data. Again, the scatter is about $5 \%$ of absolute L/D. Although the scatter bands of the later two metrics are better than those of lift and drag, they are only comparable to the scatter bands of the wing/body configurations of Case 1 .

Following the measure-of-merit developed for Case 1, we introduce a similar measure-of-merit for Case 2. However, now there are data for four levels of resolution in the grid sequences.

$$
\begin{gathered}
\mathcal{Y}_{X F}=\frac{\mathcal{X}_{F} \mathcal{Y}_{X}-\mathcal{X}_{X} \mathcal{Y}_{F}}{\mathcal{X}_{F}-\mathcal{X}_{X}}, \quad \mathcal{Y}_{F M}=\frac{\mathcal{X}_{M} \mathcal{Y}_{F}-\mathcal{X}_{F} \mathcal{Y}_{M}}{\mathcal{X}_{M}-\mathcal{X}_{F}}, \quad \mathcal{Y}_{M C}=\frac{\mathcal{X}_{C} \mathcal{Y}_{M}-\mathcal{X}_{M} \mathcal{Y}_{C}}{\mathcal{X}_{C}-\mathcal{X}_{M}} \\
\mathcal{D}(\mathcal{Y})=\left|\mathcal{Y}_{X F}-\mathcal{Y}_{F M}\right|+\left|\mathcal{Y}_{F M}-\mathcal{Y}_{M C}\right|
\end{gathered}
$$

The choice of $\mathcal{D}(\mathcal{Y})$ is intended to penalize any non-monotonic trends in the Richardson extrapolations, when performed in order of grid resolution. Definitions for $\mathcal{M O M}$ and $A v g \mathcal{M O M}$ are as before, but are now applied to the W1 and W2 data. The continuum estimates of the $\mathcal{Y}_{X F}$ extrapolations are summarized in Table VIII for the DPW-W1 wing and in Table IX for the DPW-W2 wing. Table X puts these data in rank order by $\operatorname{Avg} \mathcal{M O M}$; this is also illustrated in Figure 29. In general, these $\operatorname{Avg} \mathcal{M O M}$ values are much better behaved than those from the wing-body solutions of Case 1, and there does not appear to be any clearly-identified outliers. However, one peculiarity that does stand out in this figure is that the $\mathcal{M O} \mathcal{M}_{W 2}$ values of Ranks $05,07-10$ are noticably higher than their corresponding $\mathcal{M O M}_{W 1}$ values. Upon closer inspection, these five data blocks exclusively utilized the same structured-mesh multiblock grid family. Based on this observation, is it possible that the W2 grids of this grid family were not generated in a consistent manner? In fact, Scheidigger ${ }^{31}$ discovered a minor inconsistency with the $Y^{+}$spacing for this grid family and noted it in his presentation at the workshop. Although this inconsistency might be considered a minor one, it is interesting that it seems to be detected by our choice of $\mathcal{M O M}$. Furthermore, it is worth stating that a seemingly minor inconsistency between members of a grid family can manifest itself as a significant deviation from the expected linear grid-convergence trend line. This emphasizes the fact that it is extremely important to develop uniformally-refined, and parametrically-consistent grids of a grid family when conducting asymptotic grid convergence studies.

Figure 30 provides estimates for lift-to-drag ratios for both wings in rank order. The data of this figure unanimously show an improvement of the aerodynamic performance of DPW-W2 over that of DPW-W1. The min-max scatter band of the continuum estimates of L/D on each wing is less than $4 \%$.

\section{Case 2b: Drag Polar on Medium Grid Study}

Figures 31-32 provide the polar of idealized profile drag, $C_{D P}$, for DPW-W1 and DPW-W2, respectively. Notice that the DPW-W1 polar has a typical parabolic shape, while the DPW-W2 polar exhibits a dragbucket behavior. This characteristic is common for single-point optimizations in a low-dimension design space. In these Medium grid drag polars, at the higher lifting conditions, data block 6 consistently shows a higher idealized profile drag than that of the other data blocks.

Figures 33-34 illustrate the computed lift curves. Note the linear behavior of the DPW-W1 wing lift curve for $-1^{\circ} \leq \alpha \leq 1^{\circ}$. In this same $\alpha$ range, note the nonlinear behavior of the DPW-W2 wing lift curve. The scatter bands of both sets of lift curves is about $\Delta C_{L} \sim 0.04$ in size. The round-over of these curves occur at $\alpha \geq 1.5^{\circ}$. The lift curve of data block 6 is on the low extreme side of the set.

Figures 35-36 show the pitching-moment trends with angle-of-attack. With the exception of data block 9 , the shape of these curves are very similar. The pitching moment curve of data block 6 is on the high extreme side of these data.

A curiosity of the Case 2 results is that our choice of $A v g \mathcal{M O M}$ did not clearly identify any outliers, and yet the results of data block 6 consistently seem to be at an extreme of the scatter bands. And while the 
results of data block 6 do not warrant being classified as an outlier, it would seem that its $A v g \mathcal{M O M}$ value should be on the high side. However, in Case 1 our measure-of-merit did an exceptional job in identifing the outliers. The difference is, in Case 1 the outlier data blocks exhibited outlier traits at the flow condition where the grid-convergence study was performed. Whereas the aggragate results of Case 2 remain clustered at the flow condition utilized by the grid-convergence study, and it is only at the higher lifting conditions that data block 6 migrates to an extreme of the scatter bands.

Table VIII: Case 2 DPW-W1 Data Extrapolated To Continuum.

\begin{tabular}{|c|c|c|c|c|c|c|c|}
\hline Tag & $C_{L}$ & $C_{D}$ & $C_{D . P R}$ & $C_{D . S F}$ & $C_{M}$ & $C_{D}-C_{L}^{2} /(\pi A R)$ & $L / D$ \\
\hline \hline 1 & 0.48684 & 0.02098 & 0.01467 & 0.00631 & -0.06970 & 0.01155 & 23.21 \\
\hline 2 & 0.47626 & 0.02039 & 0.01430 & 0.00615 & -0.06740 & 0.01137 & 23.36 \\
\hline 3 & 0.46245 & 0.01978 & 0.01375 & 0.00603 & -0.06390 & 0.01127 & 23.38 \\
\hline 4 & 0.48160 & 0.02021 & 0.01422 & 0.00600 & -0.06822 & 0.01098 & 23.83 \\
\hline 5 & 0.47803 & 0.02052 & 0.01460 & 0.00592 & -0.06774 & 0.01143 & 23.30 \\
\hline 6 & 0.42668 & 0.01788 & 0.01245 & 0.00544 & -0.05470 & 0.01064 & 23.86 \\
\hline 7 & 0.45361 & 0.01915 & 0.01324 & 0.00592 & -0.06172 & 0.01096 & 23.69 \\
\hline 8 & 0.47359 & 0.01982 & 0.01398 & 0.00581 & -0.06544 & 0.01090 & 23.89 \\
\hline 9 & 0.46727 & 0.01979 & 0.01399 & 0.00580 & -0.06491 & 0.01110 & 23.61 \\
\hline 10 & 0.46564 & 0.01933 & 0.01382 & 0.00554 & -0.06466 & 0.01070 & 24.09 \\
\hline
\end{tabular}

Table IX: Case 2 DPW-W2 Data Extrapolated To Continuum.

\begin{tabular}{|c|c|c|c|c|c|c|c|}
\hline Tag & $C_{L}$ & $C_{D}$ & $C_{D . P R}$ & $C_{D . S F}$ & $C_{M}$ & $C_{D}-C_{L}^{2} /(\pi A R)$ & $L / D$ \\
\hline \hline 1 & 0.50869 & 0.02126 & 0.01498 & 0.00628 & -0.06920 & 0.01096 & 23.93 \\
\hline 2 & 0.50491 & 0.02127 & 0.01517 & 0.00610 & -0.06857 & 0.01113 & 23.74 \\
\hline 3 & 0.48817 & 0.02035 & 0.01435 & 0.00599 & -0.06434 & 0.01087 & 23.99 \\
\hline 4 & 0.50180 & 0.02044 & 0.01448 & 0.00597 & -0.06726 & 0.01043 & 24.54 \\
\hline 5 & 0.49894 & 0.02078 & 0.01491 & 0.00589 & -0.06384 & 0.01087 & 24.01 \\
\hline 6 & 0.44704 & 0.01801 & 0.01261 & 0.00540 & -0.05362 & 0.01007 & 24.82 \\
\hline 7 & 0.47263 & 0.01922 & 0.01333 & 0.00589 & -0.06003 & 0.01033 & 24.59 \\
\hline 8 & 0.49359 & 0.02009 & 0.01429 & 0.00580 & -0.06533 & 0.01040 & 24.56 \\
\hline 9 & 0.49206 & 0.02011 & 0.01437 & 0.00574 & -0.06490 & 0.01047 & 24.47 \\
\hline 10 & 0.49249 & 0.01983 & 0.01434 & 0.00550 & -0.06488 & 0.01018 & 24.84 \\
\hline
\end{tabular}

Table X: Case 2 Continuum $L / D$ Estimates Ranked By Avg $\mathcal{M O M}$.

\begin{tabular}{|c|c|c|c|c|c|c|}
\hline Rank & AvgMoM & W1-Merit & W2-Merit & Tag & W1.L/D & W2.L/D \\
\hline \hline 1 & 0.00017 & 0.00019 & 0.00015 & 7 & 23.69 & 24.59 \\
\hline 2 & 0.00026 & 0.00027 & 0.00026 & 4 & 23.83 & 24.54 \\
\hline 3 & 0.00041 & 0.00040 & 0.00042 & 8 & 23.89 & 24.56 \\
\hline 4 & 0.00073 & 0.00062 & 0.00083 & 6 & 23.86 & 24.82 \\
\hline 5 & 0.00075 & 0.00046 & 0.00104 & 10 & 24.09 & 24.84 \\
\hline 6 & 0.00076 & 0.00074 & 0.00079 & 5 & 23.30 & 24.01 \\
\hline 7 & 0.00109 & 0.00083 & 0.00134 & 9 & 23.61 & 24.47 \\
\hline 8 & 0.00139 & 0.00063 & 0.00216 & 2 & 23.36 & 23.74 \\
\hline 9 & 0.00151 & 0.00095 & 0.00207 & 3 & 23.38 & 23.99 \\
\hline 10 & 0.00161 & 0.00137 & 0.00184 & 1 & 23.21 & 23.93 \\
\hline
\end{tabular}




\section{General Observation}

A general observation, after reviewing all of the results, is that there is a set of CFD codes whose members all seem to agree relatively well with each other, and do so over all of the test cases spanning the DPW Series. Most noteworthy about this core set of codes is that it is comprised of flow solvers that are based on all types of grids. Hence, several unstructured and hybrid mesh solvers have matured sufficiently to be useful CFD tools for accurate drag predictions. Alternatively, a solver based on structured meshes does not automatically imply the same.

\section{On-Going Plans}

At the time of writing this paper, work is underway at NASA and DLR preparing for a 2007 test of a refurbished DLR-F6 model in NASA's National Transonic Facility (NTF). The goal of the test is to provide data for comparison to existing DPW-III calculations with and without the Boeing-designed side-of-body fairing and at a Reynolds number higher than in the current database. DLR is providing all model hardware, including the new side-of-body fairing and balance block to attach to the NASA balance. NASA will provide instrumentation, new model support hardware to closely match the ONERA hardware used in earlier tests, and NTF test time. The maximum Reynolds number is likely to be 5 million based on mean aerodynamic chord, due to load limitations on the model and support hardware, and because the model is not suitable for cryogenic conditions. The planned test matrix includes 3 million Reynolds number to match existing data and Mach numbers from 0.4 to 0.8. The clean-wing configuration with and without the new side-of-body fairing is included. Data to be acquired includes forces, moments, surface pressures, and wing deformations under load. Targeted flow visualization will include transition measurements via sublimating chemicals, and an as yet undetermined technique such as mini-tufts to visualize separation. This test is currently scheduled for early summer 2007.

Another collaboration currently being pursued by the organizing committee is related to the preparation of future workshops. A recurring theme of these events has consistently identified that the quality of the CFD results are only as good as the quality of the underlying meshes. The linkage between grid quality and solution quality has been recognized for decades. Nonetheless, it has been very difficult for the organizing committee to guarantee that all baseline meshes meet a consistent level of quality. To help address this issue, the committee has initiated a dialog with the AIAA Meshing, Visualization, and Computational Environments (MVCE) TC to develop a more rigorous process of quality control for the standard baseline grids. This may also result in a special session on grid generation prior to future drag prediction workshops where the baseline grids are officially made available to the public domain, and quality-metrics of these grids are formally documented and discussed in an open forum.

\section{Conclusions}

Results from the Third AIAA Drag Prediction Workshop (DPW-III) are summarized. This workshop focused on the prediction of drag for wing-body and wing-alone configurations that are representative of transonic transport aircraft. Numerical calculations were performed using industry-relevant test cases. $\mathrm{Nu}-$ merous Reynolds-Averaged Navier-Stokes CFD results on fully-turbulent flows are provided. These solutions are performed on structured, unstructured, and hybrid grid systems. The structured grid sets include pointmatched multi-block meshes and over-set grid systems. The unstructured and hybrid grid sets are comprised of tetrahedral, pyramid, and prismatic elements. Effort was made to provide a high-quality and parametrically consistent family of grids for each grid type about each configuration under study. The wing-body families are comprised of a coarse, medium, and fine grid, while the wing-alone families also include an extra-fine mesh. These mesh sequences are utilized to help determine how the provided flow solutions fair with respect to asymptotic grid convergence, and are used to estimate an absolute drag of each configuration.

The DPW Series has provided a very broad view of the state-of-the-art of CFD applications within the industry, much more so than that which can be garnered by an isolated study. In fact, by reviewing in isolation any one of the DPW-III's individual data blocks, one may arrive at different conclusions than those presented herein. For example, a typical publication may show how successful a CFD solution matches test data. By combining a large set of solutions from many sources around the world, this workshop clearly shows that there remains much room for improvement. While this conclusion is somewhat disappointing, it is tempered by an observation that there exists a core set of CFD methods that consistently agree with each 
other in general, and do so on all test cases spanning the workshop series. Most noteworthy about this core set of solvers is that these methods are based on all grid types.

A measure-of-merit is introduced that quantifies issues specifically associated with grid-convergence data. This metric is not biased by grid type, absolute value of drag, or slope of grid-convergence trend line. It does not identify the source of a problem, only that a problem exists with the grid-convergence data. Applied to the data blocks of DPW-III, this metric successfully identified which data blocks were outliers, at least at the flow condition of the grid-convergence studies.

Through the data compiled by this workshop, it is obvious that several problematic issues continue to persist in the processes used for accurate drag prediction. Generating a consistent set of grids for the purpose of grid-convergence studies remains a challenge, especially for unstructured meshes. The side-ofbody separation bubble of the DLR-F6 wing/body configuration continues to be a source of difficulty; the full set of CFD solutions show a large variation of predicted bubble sizes. However, on a good note, the skin-friction predictions of the aggragate data blocks are well behaved and form relatively tight groupings.

An underlying objective of the DPW-III was to test a hypothesis that pockets of flow separation can be a root cause of poor grid convergence characteristics. While it appears that pockets of flow separation did adversely affect the grid-convergence trends of many of the CFD data blocks provided by the participants, it did not seem to cause issues with others. This is somewhat of an unexpected finding of the DPW-III.

\section{Acknowledgments}

The authors thank the AIAA Applied Aerodynamics Technical Committee for sponsoring the Drag Prediction Workshop Series. We also thank our respective organizations for their continued support in this endeavor. A special thanks is extended to the participants of DPW-III, for without their contributions, this workshop would not have been possible. Finally, the planning of these workshops throughout the duration of the series has been conducted by a substantial number of dedicated individuals. Members of the aggragate organizing committees include: Shreekant Agrawal, Olaf Brodersen, Bob Dowgwillo, Bernhard Eisfeld, Jean Luc Godard, Mike Hemsch, Steve Klausmeyer, Kelly Laflin, Dave Levy, Mori Mani, Rick Matus, Dimitri Mavriplis, Joe Morrison, Bas Oskam, Shahyar Pirzadeh, Mark Rakowitz, Ed Tinoco, John Vassberg, Rich Wahls, and Tom Zickuhr.

\section{References}

\footnotetext{
${ }_{1}^{1} 1^{s t}$ AIAA CFD Drag Prediction Workshop. http://aaac.larc.nasa.gov/tsab/cfdlarc/aiaa-dpw/Workshop1/workshop1.html, June 2001.

${ }^{2}$ G. Redeker. DLR-F4 wing-body configuration. In A Selection of Experimental Test Cases for the Validation of CFD Codes, number $A R-303$, pages B4.1-B4.21. AGARD, August 1994.

${ }^{3}$ J. C. Vassberg, M. A. DeHaan, and A. J. Sclafani. Grid generation requirements for accurate drag predictions based on OVERFLOW calculations. AIAA Paper 2003-4124, $16^{\text {th }}$ AIAA Computational Fluid Dynamics Conference, Orlando, FL, June 2003.

${ }^{4}$ D. W. Levy, J. C. Vassberg, R. A. Wahls, T. Zickuhr, S. Agrawal, S. Pirzadeh, and M. J. Hemsch. Summary of data from the first AIAA CFD Drag Prediction Workshop. AIAA paper 2002-0841, Reno, NV, January 2002.

${ }^{5}$ D. W. Levy, J. C. Vassberg, R. A. Wahls, T. Zickuhr, S. Agrawal, S. Pirzadeh, and M. J. Hemsch. Summary of data from the first AIAA CFD Drag Prediction Workshop. AIAA Journal of Aircraft, 40(5):875-882, Sep-Oct 2003.

${ }^{6}$ M. J. Hemsch. Statistical analysis of cfd solutions from the Drag Prediction Workshop. AIAA paper 2002-0842, Reno, NV, January 2002.

${ }^{7}$ M. Hemsch. Statistical analysis of CFD solutions from the drag prediction workshops. In CFD-based Aircraft Drag Prediction and Reduction, Hampton, VA, November 2003. von Karman Institue Lecture Series.

${ }^{8}$ M. Rakowitz, B. Eisfeld, D. Schwamborn, and M. Sutcliffe. Structured and unstructured computations on the DLR-F4 wing-body configuration. AIAA paper 2002-0837, Reno, NV, January 2002.

${ }^{9}$ M. Rakowitz, B. Eisfeld, D. Schwamborn, and M. Sutcliffe. Structured and unstructured computations on the DLR-F4 wing-body configuration. AIAA Journal of Aircraft, 40(2):256-264, 2003.

${ }^{10}$ D. J. Mavriplis and D. W. Levy. Transonic drag predictions using an unstructured multigrid solver. AIAA paper 2002-0838, Reno, NV, January 2002.

${ }^{11}$ S. Z. Pirzadeh and N. T. Frink. Assessment of the unstructured grid software TetrUSS for drag prediction of the DLR-F4 configuration. AIAA paper 2002-0839, Reno, NV, January 2002.

${ }^{12}$ J. C. Vassberg, P. G. Buning, and C. L. Rumsey. Drag prediction for the DLR-F4 wing/body using OVERFLOW and CFL-3D on an overset mesh. AIAA Paper 2002-0840, 40th AIAA Aerospace Sciences Meeting \& Exhibit, Reno, NV, January 2002.

${ }^{13} 2^{\text {nd }}$ AIAA CFD Drag Prediction Workshop. http://aaac.larc.nasa.gov/tsab/cfdlarc/aiaa-dpw/, dpw@cessna.textron.com, June 2003.
} 
${ }^{14}$ K. R. Laflin, J. C. Vassberg, R. A. Wahls, J. H. Morrison, O. Brodersen, M. Rakowitz, E. N. Tinoco, and J. Godard. Summary of data from the second AIAA CFD drag prediction workshop. AIAA Paper 2004-0555, 42 ${ }^{\text {nd }}$ AIAA Aerospace Sciences Meeting and Exhibit, Reno, NV, January 2004.

${ }^{15}$ K. R. Laflin, J. C. Vassberg, R. A. Wahls, J. H. Morrison, O. Brodersen, M. Rakowitz, E. N. Tinoco, and J. Godard. Summary of data from the second AIAA CFD drag prediction workshop. AIAA Journal of Aircraft, 42(5):1165-1178, 2005.

${ }^{16} \mathrm{M}$. Hemsch and J. Morrison. Statistical analysis of CFD solutions from $2^{\text {nd }}$ drag prediction workshop. AIAA Paper 20040556, 42 $2^{\text {nd }}$ AIAA Aerospace Sciences Meeting and Exhibit, Reno, NV, January 2004.

${ }^{17}$ N. Pfeiffer. Reflections on the second drag prediction workshop. AIAA Paper 2004-0557, 42 ${ }^{\text {nd }}$ AIAA Aerospace Sciences Meeting and Exhibit, Reno, NV, January 2004.

${ }^{18}$ O. Brodersen, M. Rakowitz, S. Amant, P. Larrieu, D. Destarac, and M. Sutcliffe. Airbus, ONERA, and DLR results from the $2^{\text {nd }}$ AIAA drag prediction workshop. AIAA Paper 2004-0391, 42 $2^{\text {nd }}$ AIAA Aerospace Sciences Meeting and Exhibit, Reno, NV, January 2004.

${ }^{19}$ O. P. Brodersen, M. Rakowitz, S. Amant, P. Larrieu, D. Destarac, and M. Suttcliffe. Airbus, ONERA and DLR results from the second AIAA drag prediction workshop. AIAA Journal of Aircraft, 42(4):932-940, 2005.

${ }^{20}$ R. B. Langtry, M. Kuntz, and F. Menter. Drag prediction of engine-airframe interference effects with CFX-5. AIAA Paper 2004-0392, 42 ${ }^{\text {nd }}$ AIAA Aerospace Sciences Meeting and Exhibit, Reno, NV, January 2004.

${ }^{21}$ R. B. Langtry, M. Kuntz, and F. Menter. Drag prediction of engine-airframe interference effects with CFX-5. AIAA Journal of Aircraft, 42(6):1523-1529, 2005.

${ }^{22}$ A. J. Sclafani, M. A. DeHaan, and J. C. Vassberg. OVERFLOW drag predictions for the DLR-F6 transport configuration: A DPW-II case study. AIAA Paper 2004-0393, $42^{\text {nd }}$ AIAA Aerospace Sciences Meeting and Exhibit, Reno, NV, January 2004.

${ }^{23}$ C. Rumsey, M. Rivers, and J. Morrison. Study of CFD variations on transport configurations from the $2^{\text {nd }}$ AIAA drag prediction workshop. AIAA Paper 2004-0394, 42 ${ }^{\text {nd }}$ AIAA Aerospace Sciences Meeting and Exhibit, Reno, NV, January 2004.

${ }^{24} \mathrm{~K}$. Wutzler. Aircraft drag prediction using Cobalt. AIAA Paper 2004-0395, 42 ${ }^{\text {nd }}$ AIAA Aerospace Sciences Meeting and Exhibit, Reno, NV, January 2004.

${ }^{25}$ G. May, E. van der Weide, A. Jameson, and S. Shankaran. Drag prediction of the DLR-F6 configuration. AIAA Paper 20040396, 42 ${ }^{\text {nd }}$ AIAA Aerospace Sciences Meeting and Exhibit, Reno, NV, January 2004

${ }^{26}$ Y. Kim, S. Park, and J. Kwon. Drag prediction of DLR-F6 using the turbulent Navier-Stokes calculations with multigrid. AIAA Paper 2004-0397, 42 ${ }^{\text {nd }}$ AIAA Aerospace Sciences Meeting and Exhibit, Reno, NV, January 2004.

${ }^{27} \mathrm{~K}$. Yamamoto, A. Ochi, E. Shima, and R. Takaki. CFD sensitivity to drag prediction on DLR-F 6 configuration by structured method and unstructured method. AIAA Paper 2004-0398, 42 ${ }^{\text {nd }}$ AIAA Aerospace Sciences Meeting and Exhibit, Reno, NV, January 2004.

${ }^{28}$ E. Tinoco and T. Su. Drag prediction with the Zeus/CFL3D system. AIAA Paper 2004-0552, 42 ${ }^{\text {nd }}$ AIAA Aerospace Sciences Meeting and Exhibit, Reno, NV, January 2004.

${ }^{29} \mathrm{~S}$. Klausmeyer. Drag, lift, and moment estimates for transonic aircraft using the Navier-Stokes equations. AIAA $P a-$ per 2004-0553, 42 ${ }^{\text {nd }}$ AIAA Aerospace Sciences Meeting and Exhibit, Reno, NV, January 2004.

${ }^{30}$ E. Lee-Rausch, N. Frink, W. Milholen, and D. Mavriplis. Transonic drag prediction using unstructured grid solvers. AIAA Paper 2004-0554, 42 $2^{\text {nd }}$ AIAA Aerospace Sciences Meeting and Exhibit, Reno, NV, January 2004.

${ }^{31} 3^{r d}$ AIAA CFD Drag Prediction Workshop. http://aaac.larc.nasa.gov/tsab/cfdlarc/aiaa-dpw/, dpw@cessna.textron.com, June 2006 .

32 J. C. Vassberg, A. J. Sclafani, and M. A. DeHaan. A wing-body fairing design for the DLR-F6 model: a DPW-III case study. AIAA Paper 2005-4730, AIAA 23 ${ }^{\text {rd }}$ Applied Aerodynamics Conference, Toronto, Canada, June 2005.

${ }^{33}$ T. J. Baker. Mesh generation: Art or science? Progress in Aerospace Sciences, 41:29-63, 2005.

${ }^{34}$ M. D. Salas. Digital flight: The last CFD aeronautical grand challenge. Journal of Scientific Computing, Vol. 28, No. 213, September 2006.

${ }^{35} \mathrm{O}$. Brodersen and A. Sturmer. Drag prediction of engine-airframe interference effects using unstructured Navier-Stokes calculations. AIAA Paper 2001-2414, 19 ${ }^{t h}$ AIAA Applied Aerodynamics Conference, Anaheim, CA, June 2001.

${ }^{36}$ S. S. Samant, J. E. Bussoletti, F. T. Johnson, R. H. Burkhart, B. L. Everson, R. G. Melvin, and D. P. Young. TRANAIR: A computer code for transonic analysis of arbitrary configurations. AIAA Paper 87-0034, AIAA Aerospace Sciences Meeting and Exhibit, Reno, NV, January 1987.

${ }^{37}$ P. E. Gill, W. Murray, M. A. Saunders, and M. H. Wright. User's guide for npsol (version 4.0): A fortran package for nonlinear programming. Technical Report SOL 86-2, Department of Operations Research, Stanford University, Jan. 1986.

${ }^{38}$ P. E. Gill, W. Murray, M. A. Saunders, and M. H. Wright. Some theoretical properties of an augmented Lagrangian merit function. In Advances in Optimization and Parallel Computing. P. M. Pardalos (ed.), North-Holland, Amsterdam, 101-128, 1992.

${ }^{39}$ E. N. Tinoco, C. Winkler, M. Mani, and V. Venkatakrishnan. Structured and unstructured solvers for the $3^{\text {rd }}$ CFD drag prediction workshop. AIAA Paper 2007-0255, 45 ${ }^{\text {th }}$ AIAA Aerospace Sciences Meeting and Exhibit, Reno, NV, January 2007.

${ }^{40}$ A. J. Sclafani, J. C. Vassberg, N. A. Harrison, M. A. DeHaan, C. L. Rumsey, S. M. Rivers, and J. H. Morrison. Drag predictions for the DLR-F6 wing/body and DPW wings using CFL3D and OVERFLOW on an overset mesh. AIAA Paper 20070257, $45^{t h}$ AIAA Aerospace Sciences Meeting and Exhibit, Reno, NV, January 2007.

${ }^{41}$ D. J. Mavriplis. Results from the $3^{\text {rd }}$ drag prediction workshop using the NSU3D unstructured mesh solver. AIAA Paper 2007-0256, 45 th AIAA Aerospace Sciences Meeting and Exhibit, Reno, NV, January 2007.

${ }^{42}$ O. Brodersen, B. Eisfeld, J. Raddatz, and P. Frohnapfel. DLR results from the third AIAA CFD drag prediction workshop. AIAA Paper 2007-0259, 45 th AIAA Aerospace Sciences Meeting and Exhibit, Reno, NV, January 2007.

${ }^{43} \mathrm{~J}$. H. Morrison and M. J. Hemsch. Statistical analysis of CFD solutions from the third AIAA drag prediction workshop. AIAA Paper 2007-0254, 45 ${ }^{\text {th }}$ AIAA Aerospace Sciences Meeting and Exhibit, Reno, NV, January 2007. 


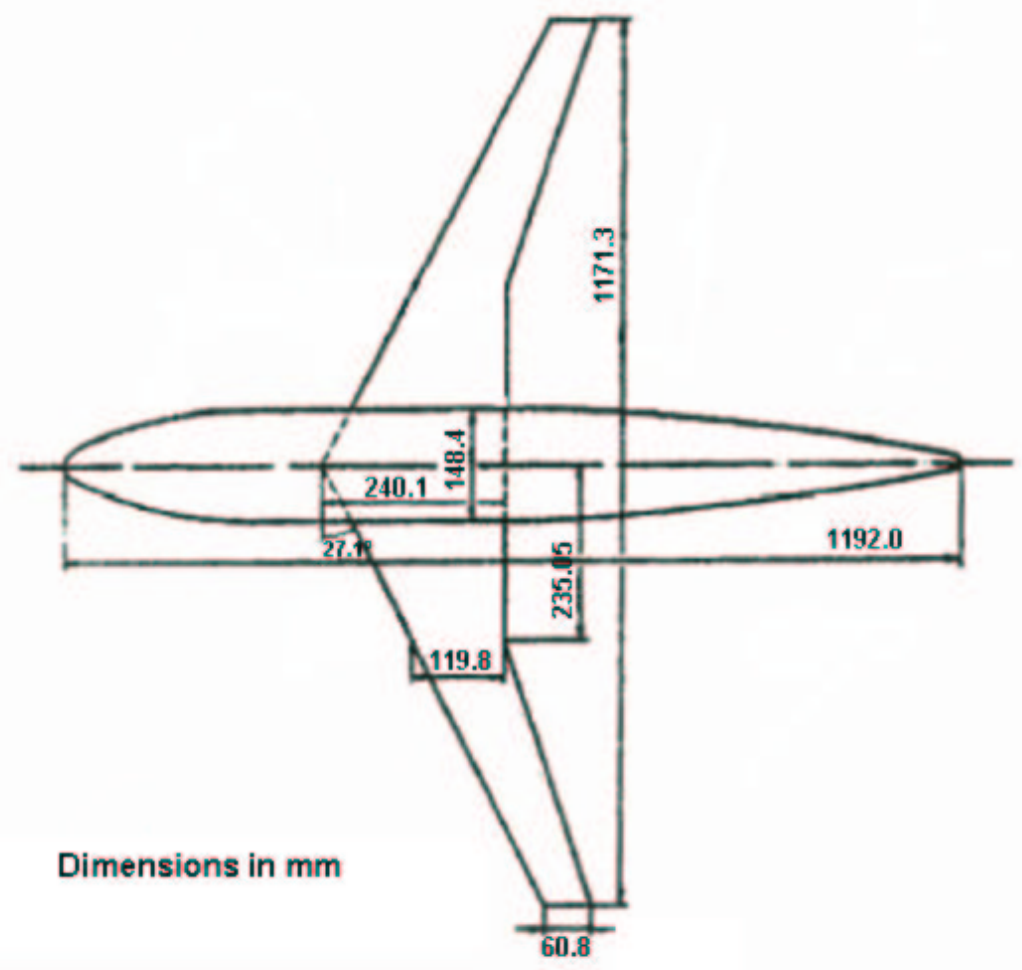

Figure 1. DLR-F6 Wing/Body Planform.
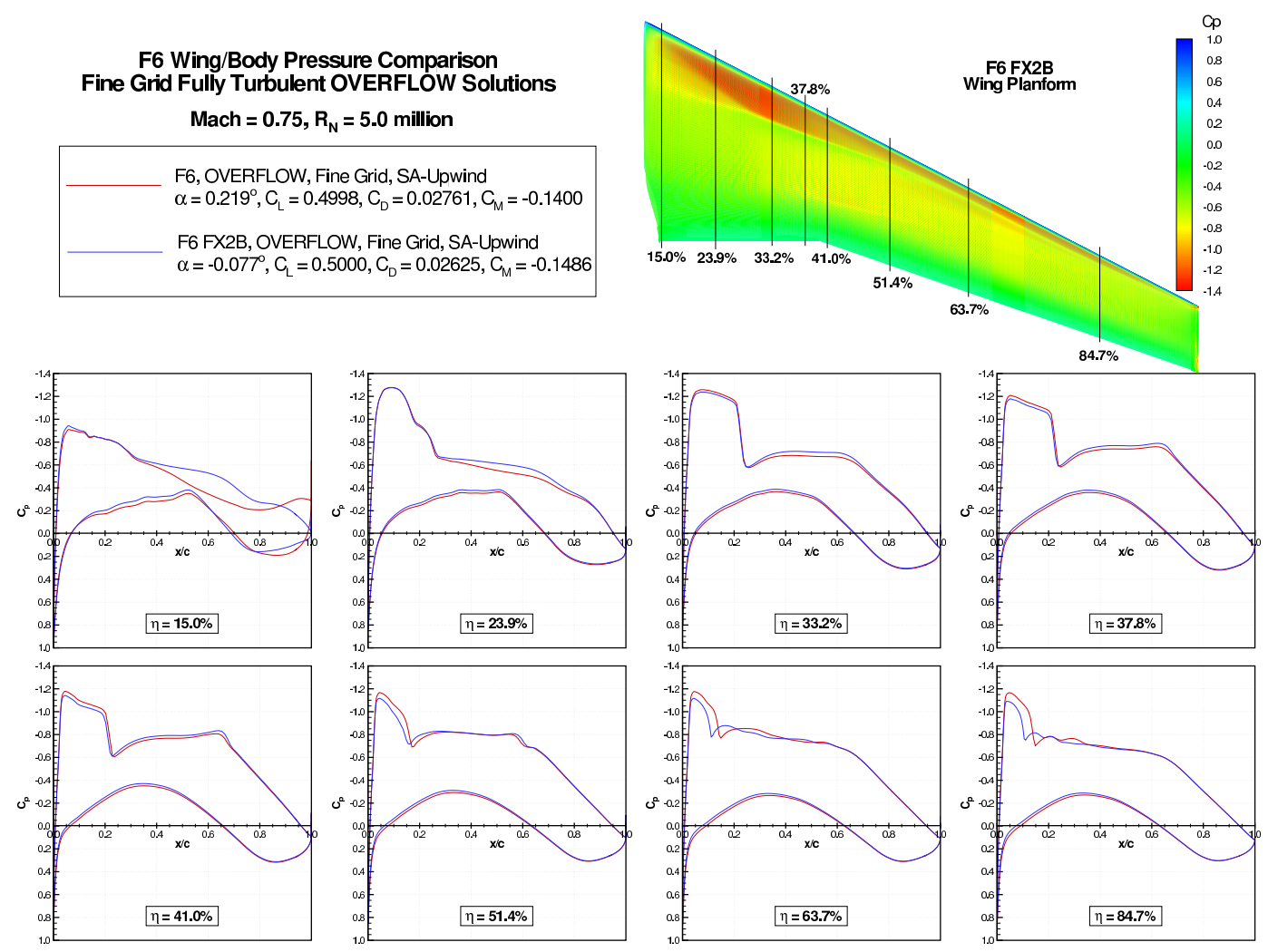

Figure 2. Comparison of Pressure Distributions on the DLR-F6 Wing/Body: $M=0.75, C_{L}=0.5, R e=5$ million. 


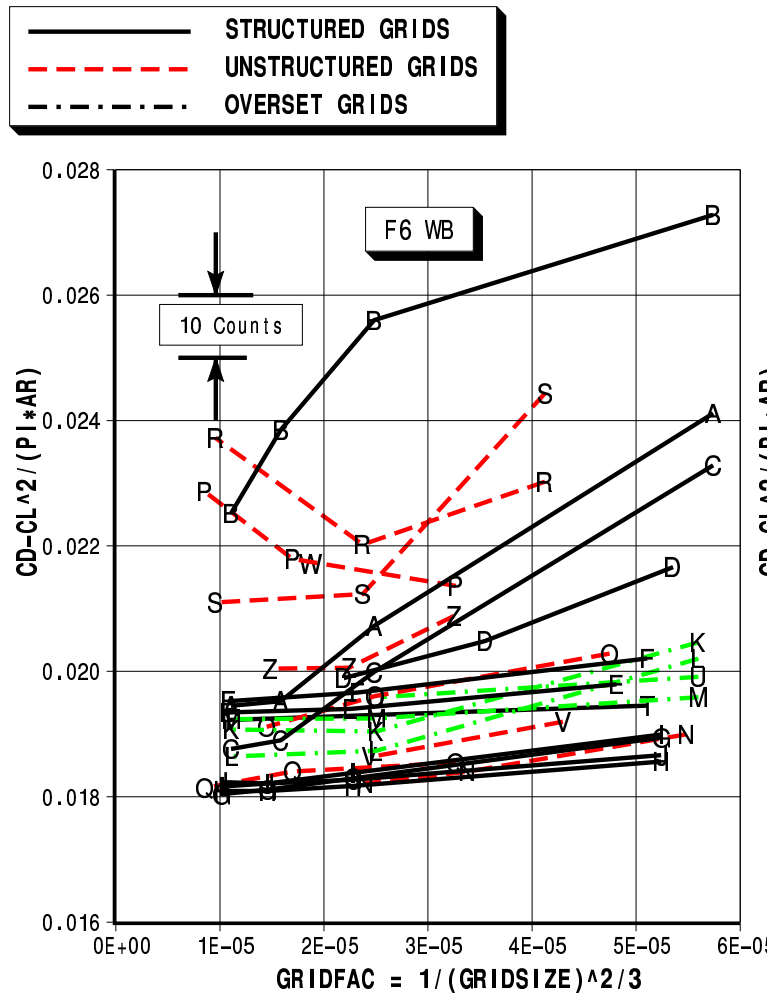

F6 Wing-Body w/wo FX2B, MACH $=0.75$

Re $=5$ Million, Fixed $C L=0.50$

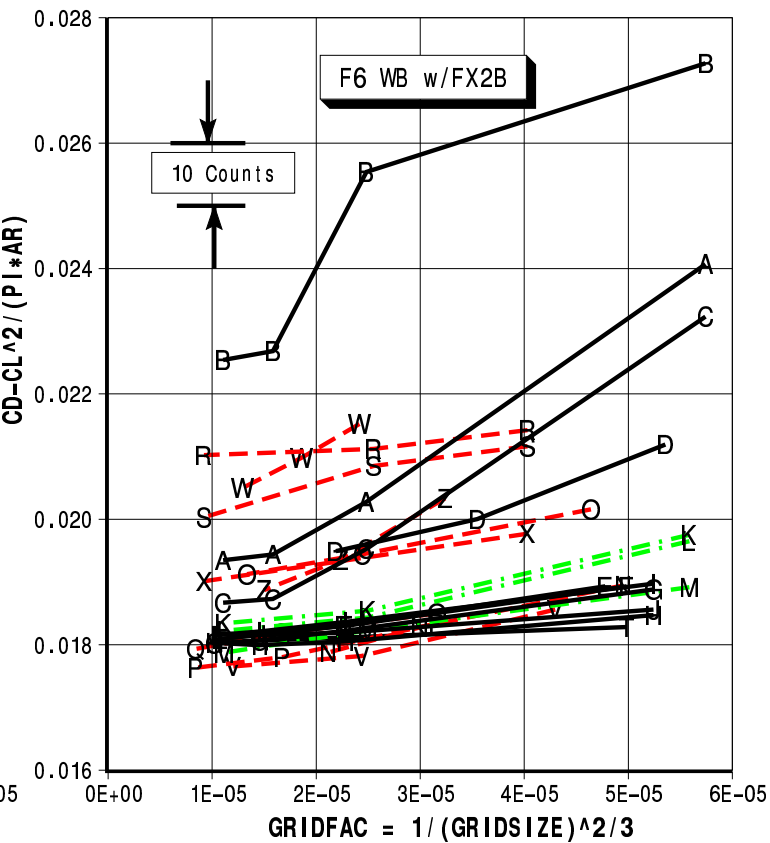

Figure 3. Case 1 Grid Sensitivity on Idealized Proflle Drag: $M=0.75, C_{L}=0.5, R e=5$ million.
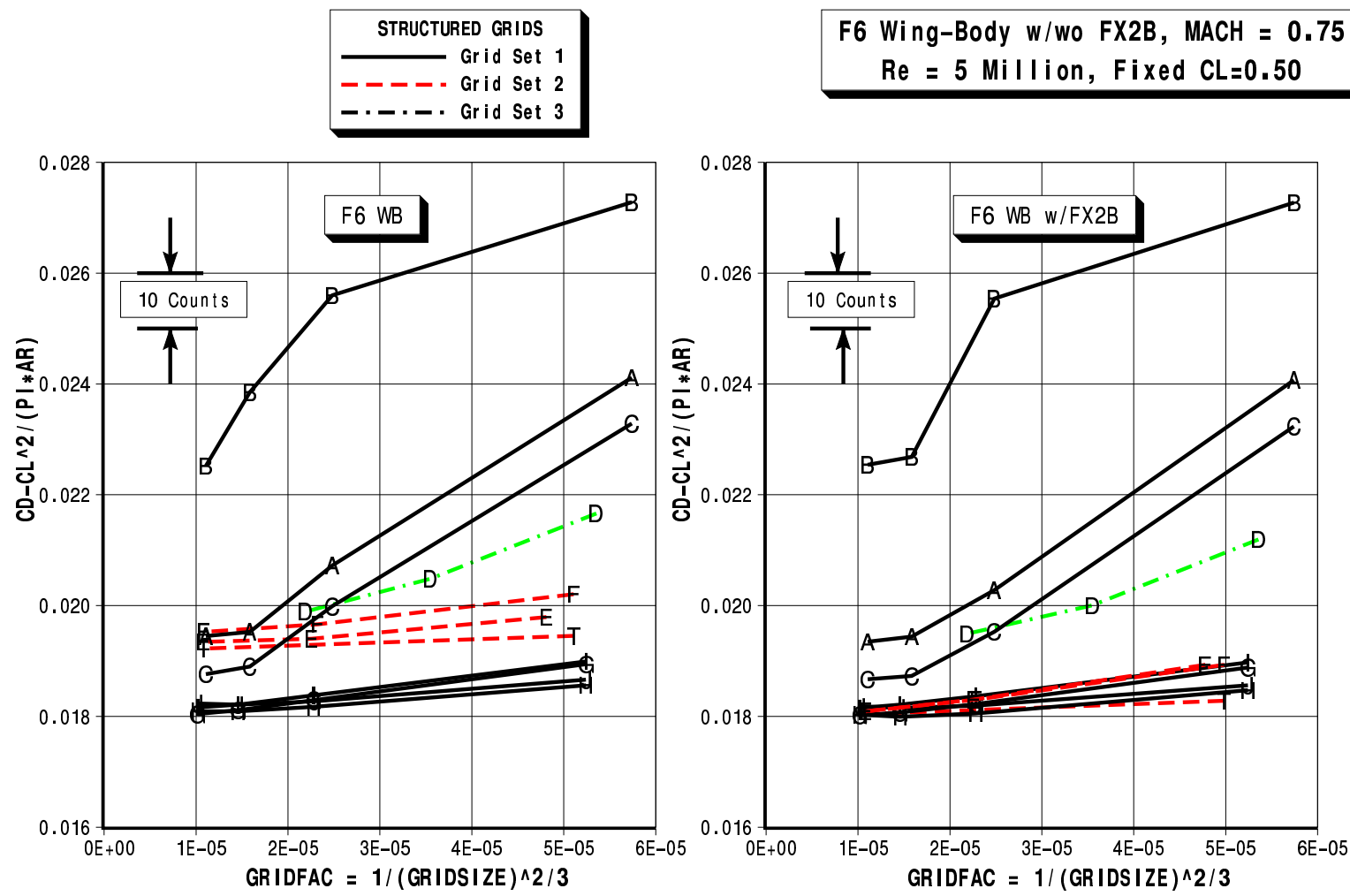

Figure 4. Case 1 Grid Sensitivity on Structured Mesh Idealized Profle Drag. 


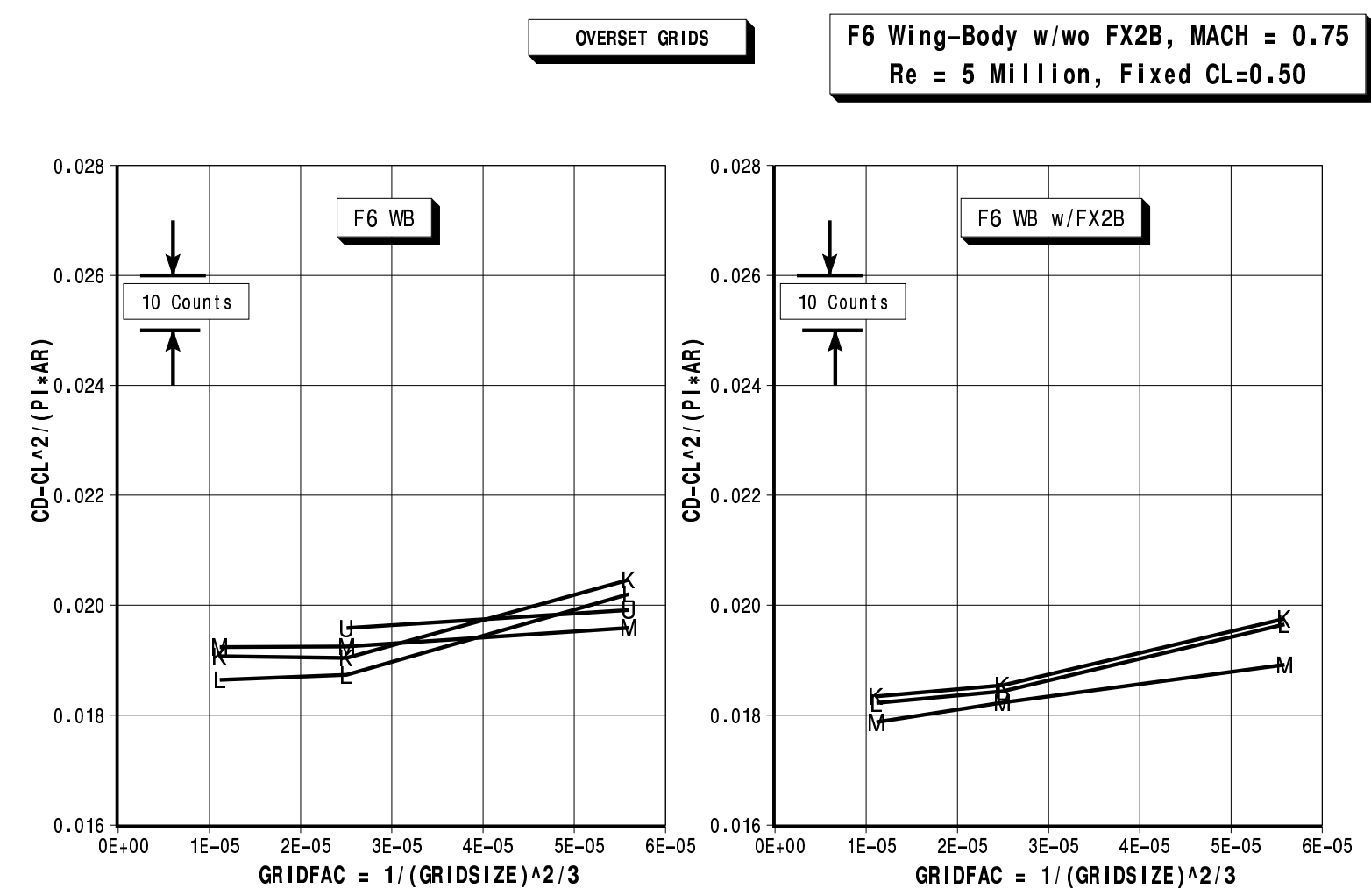

Figure 5. Case 1 Grid Sensitivity on Overset Mesh Idealized Profle Drag.

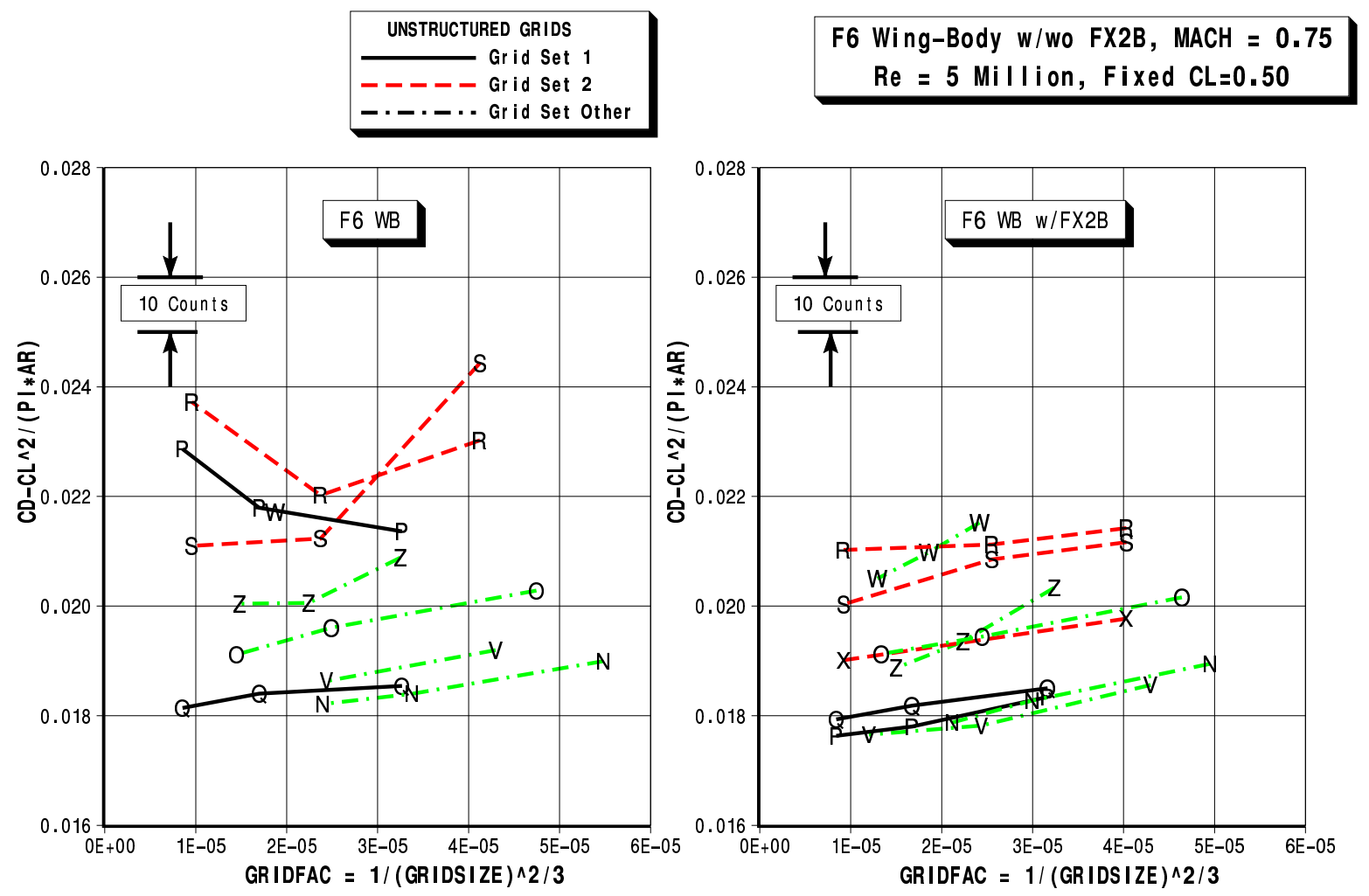

Figure 6. Case 1 Grid Sensitivity on Unstructured Mesh Idealized Profile Drag. 

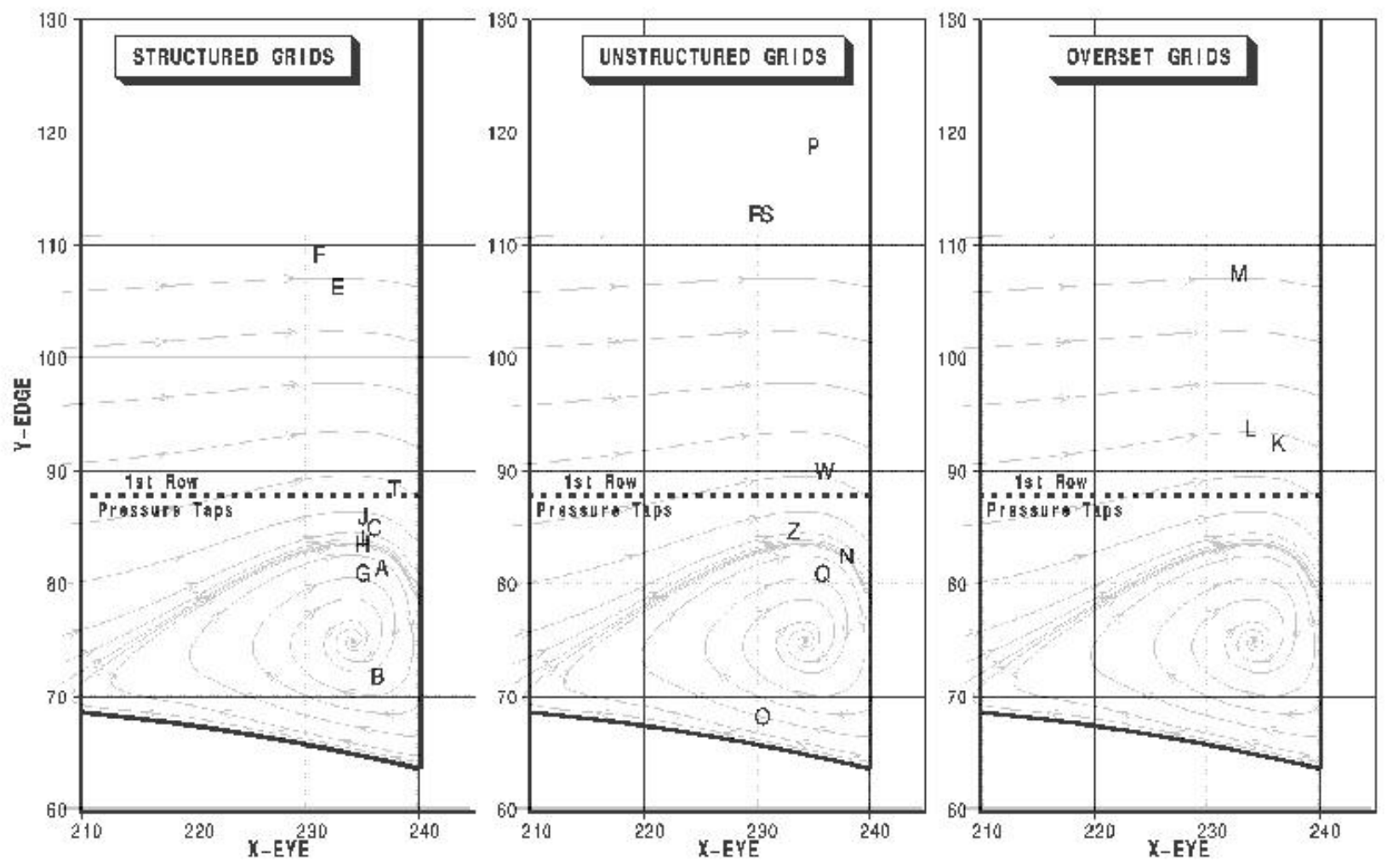

Figure 7. Case 1 Side-of-Body Separation Bubble Characteristics of the Baseline DLR-F6.

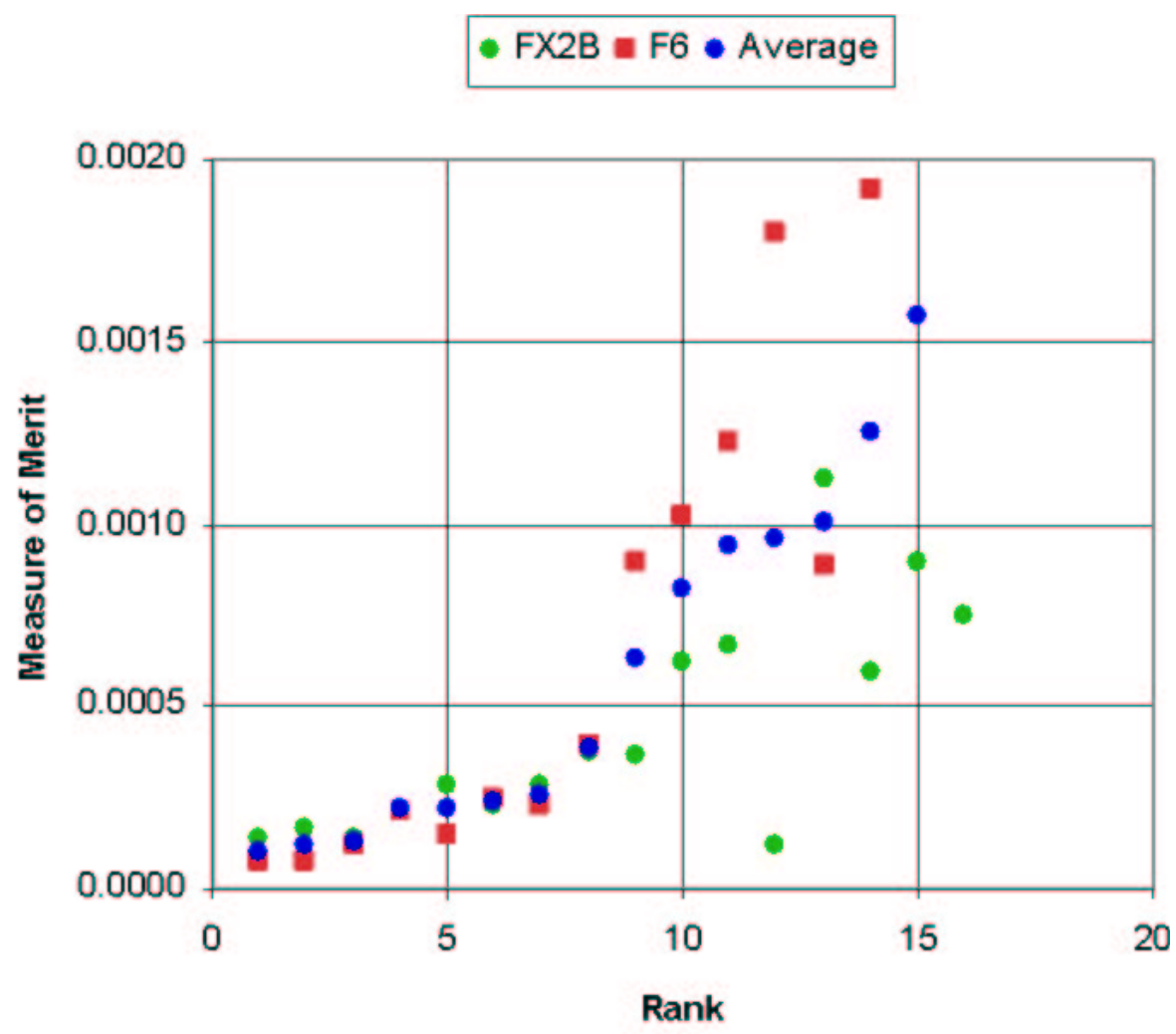

Figure 8. Case 1 Ranked by Measure of Merit. 


\section{- FX2B $\square \mathrm{F} 6$}

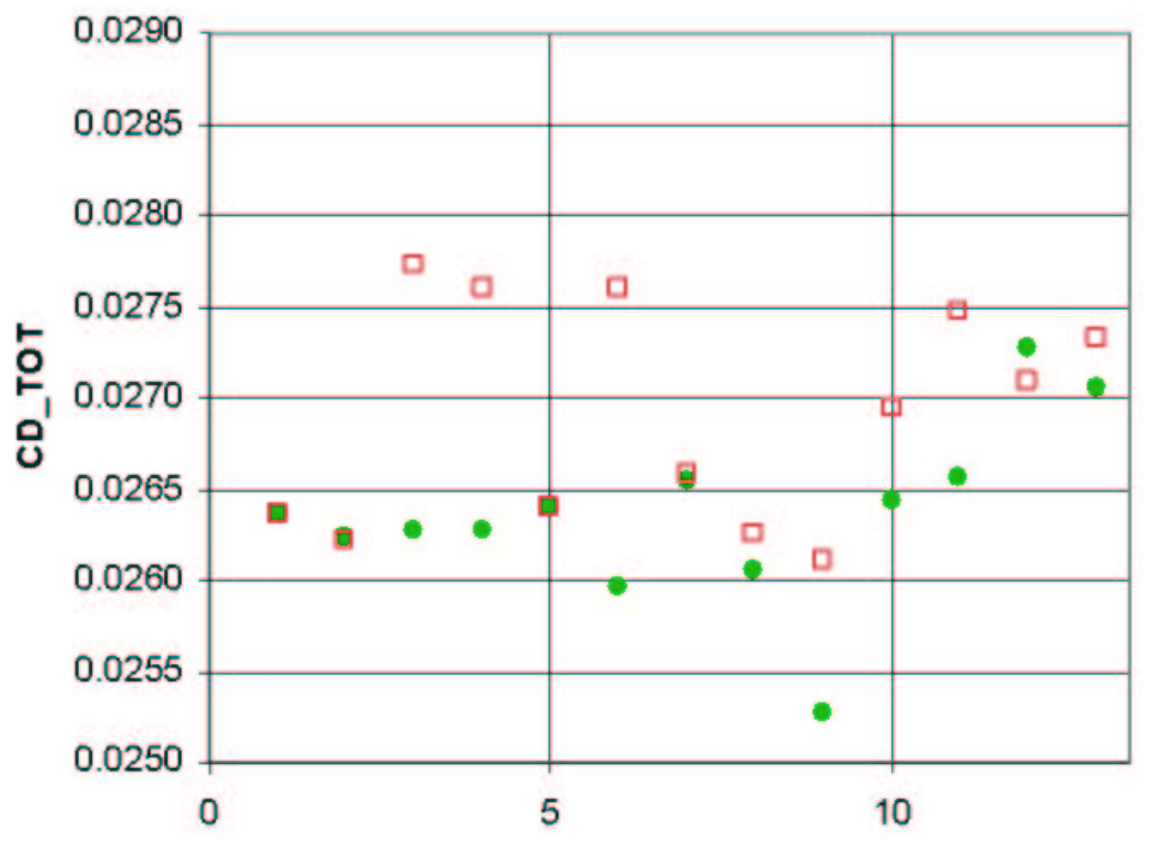

Rank

Figure 9. Case 1 Ranked Continuum Total Drag Coefficients.

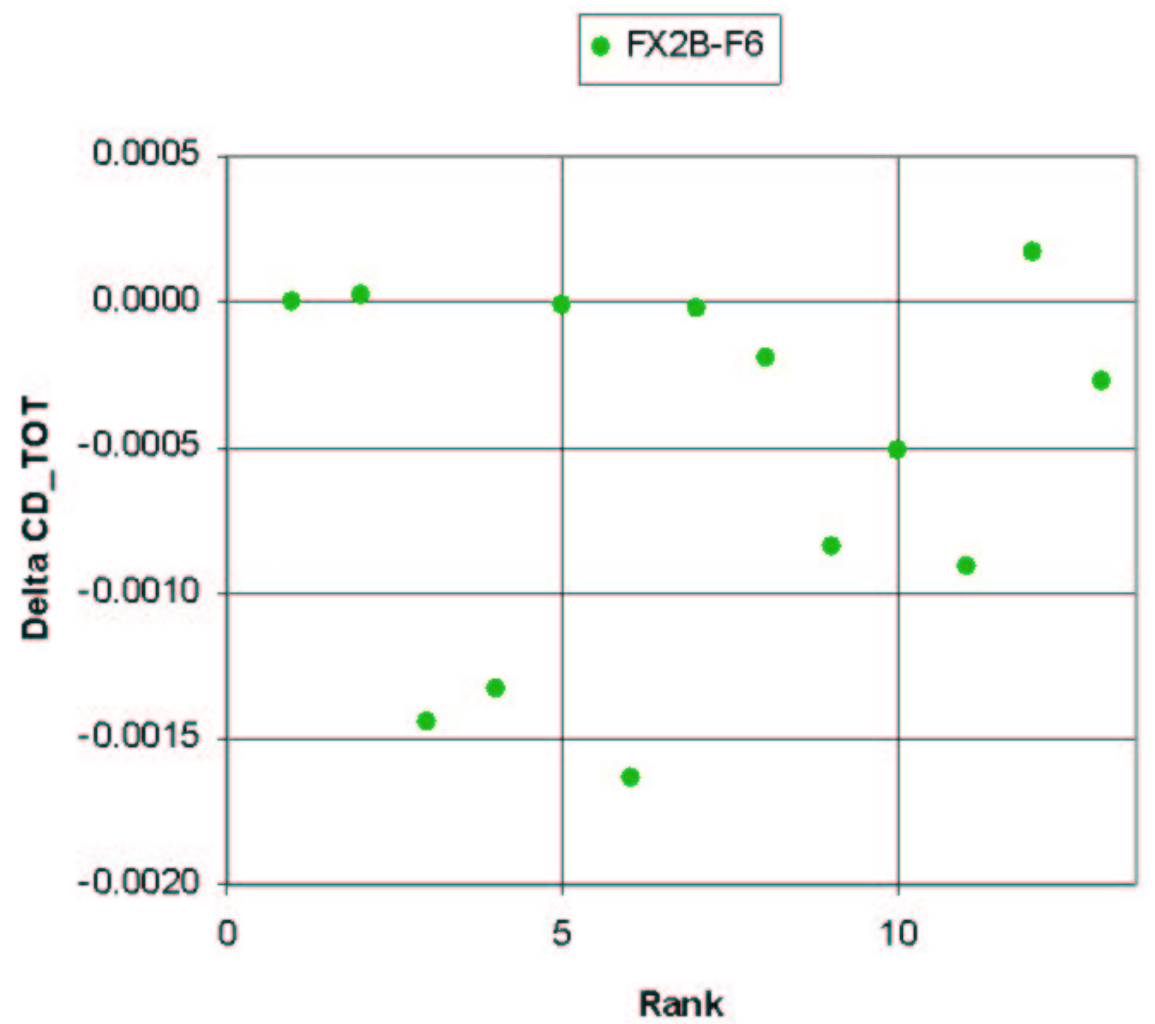

Figure 10. Case 1 Ranked Continuum Delta Drag. 


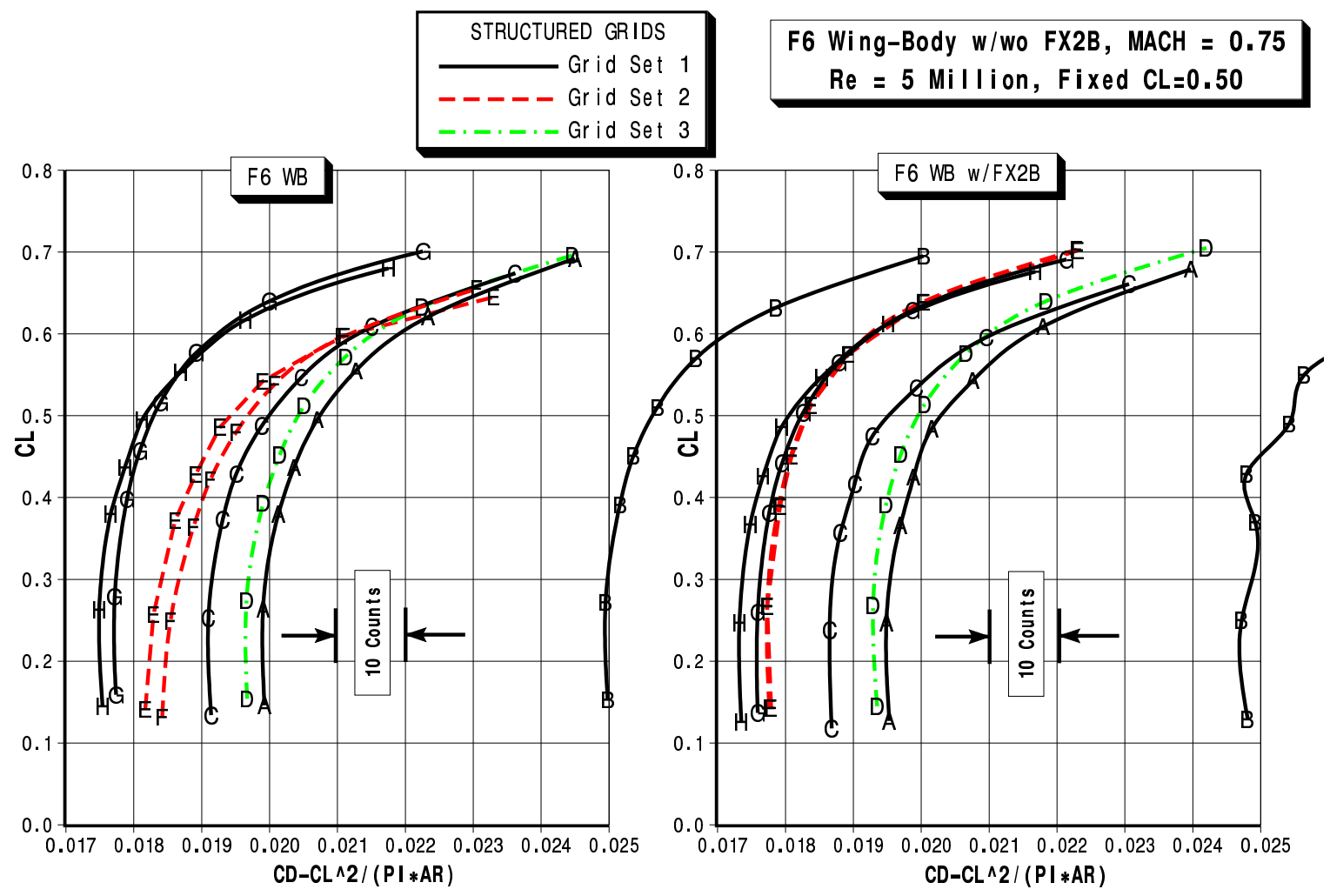

Figure 11. Case 1 Structured Mesh Idealized Profle Drag Polars.

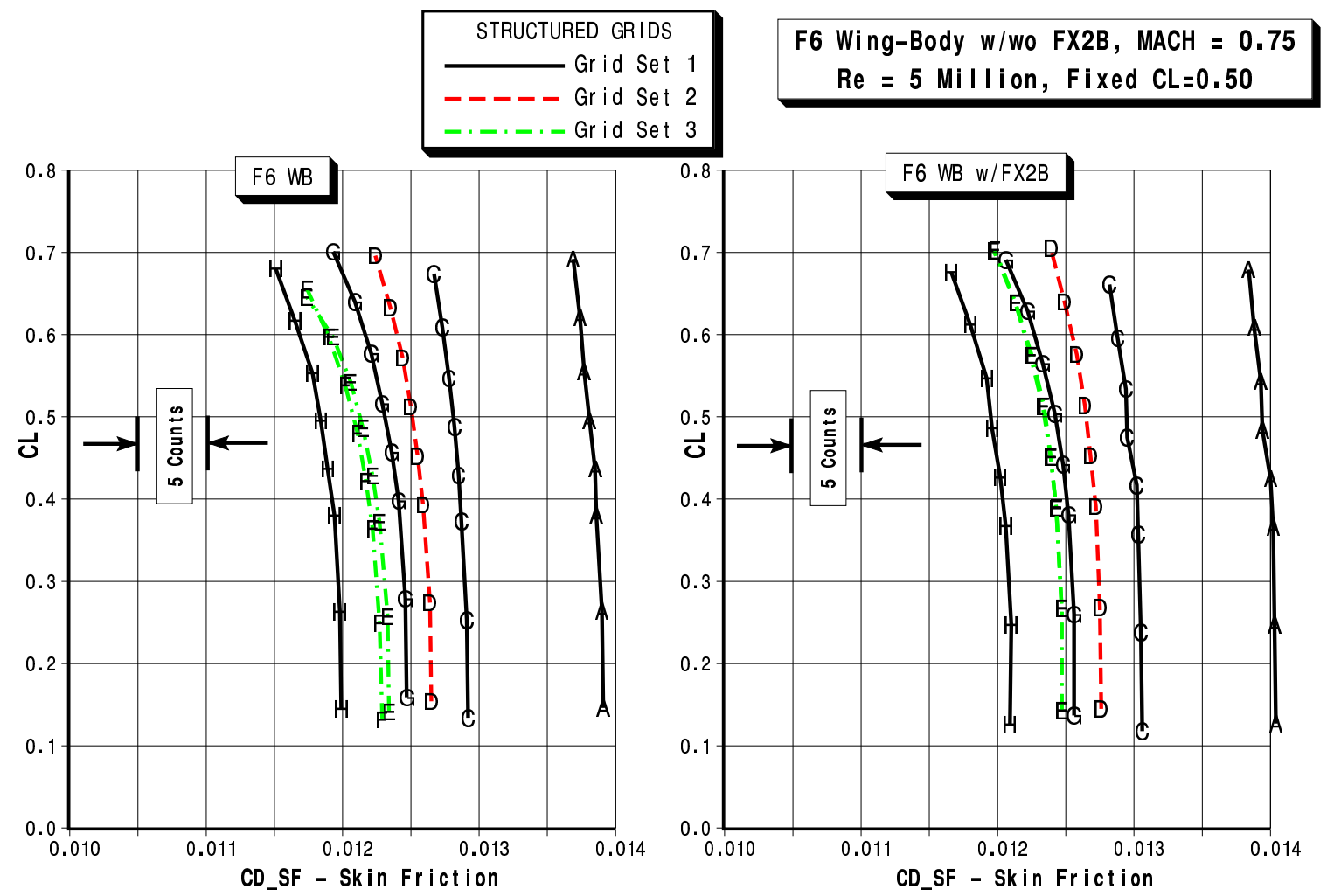

Figure 12. Case 1 Structured Mesh Skin-Friction Polars. 


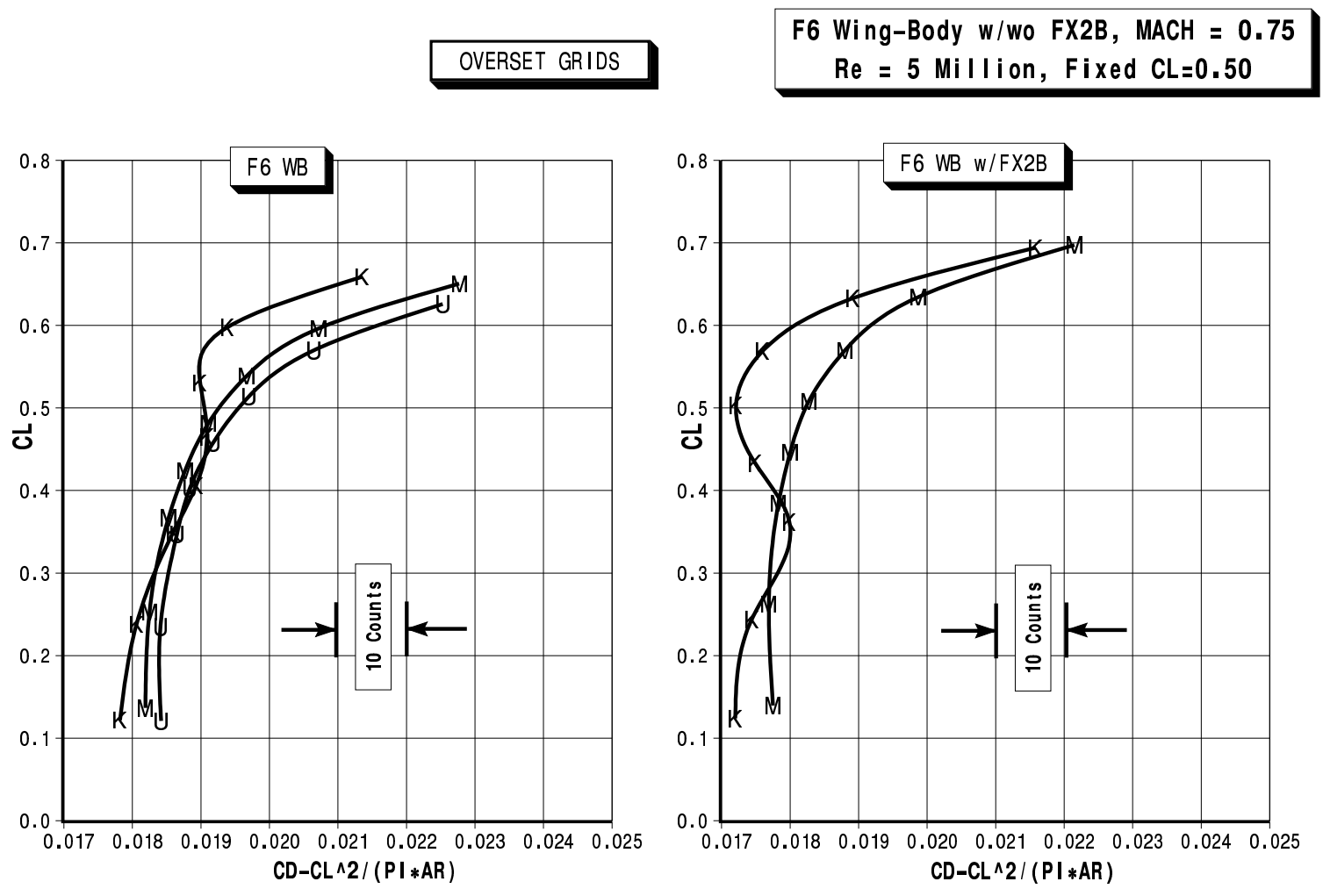

Figure 13. Case 1 Overset Mesh Idealized Proflle Drag Polars.

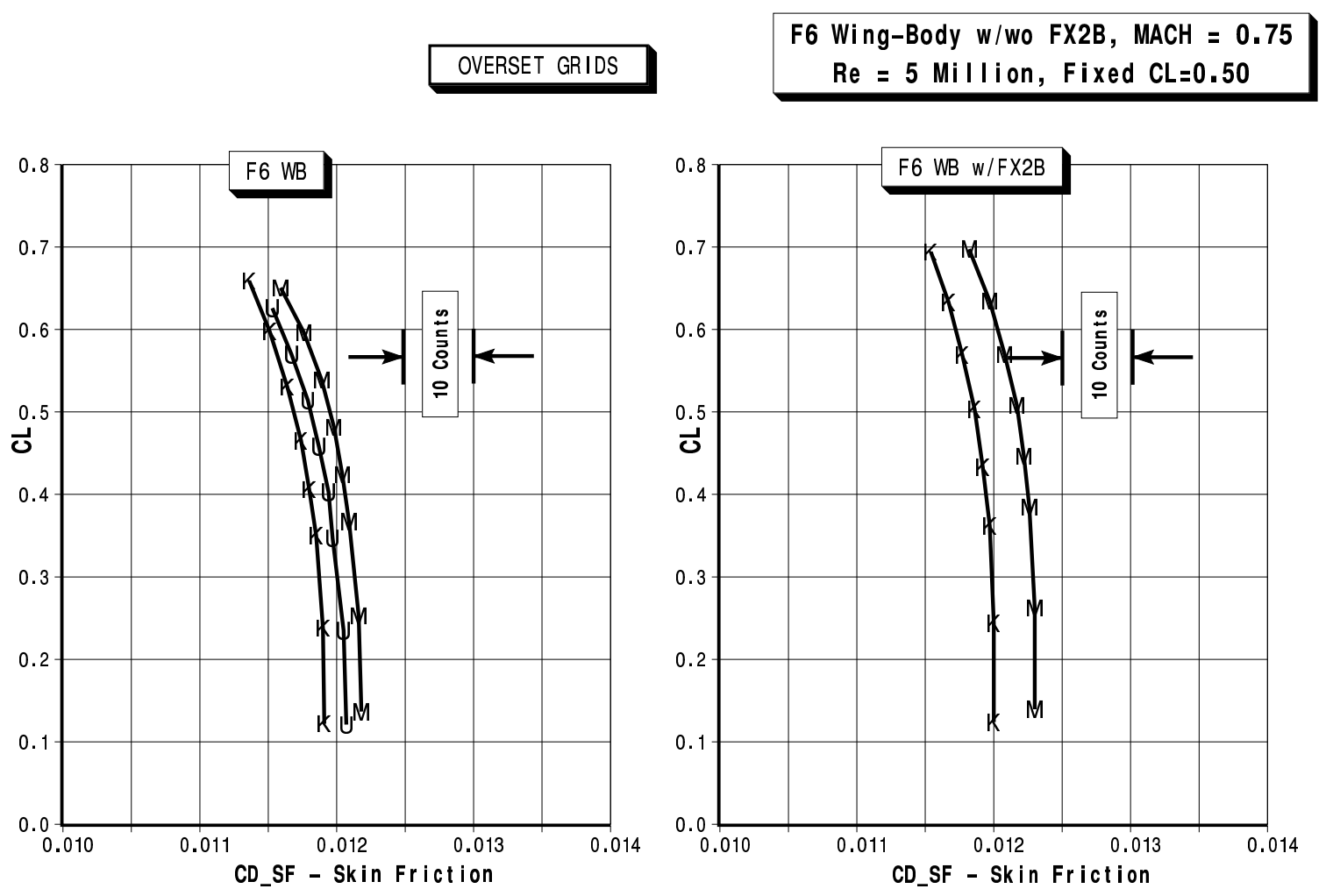

Figure 14. Case 1 Overset Mesh Skin-Friction Polars. 


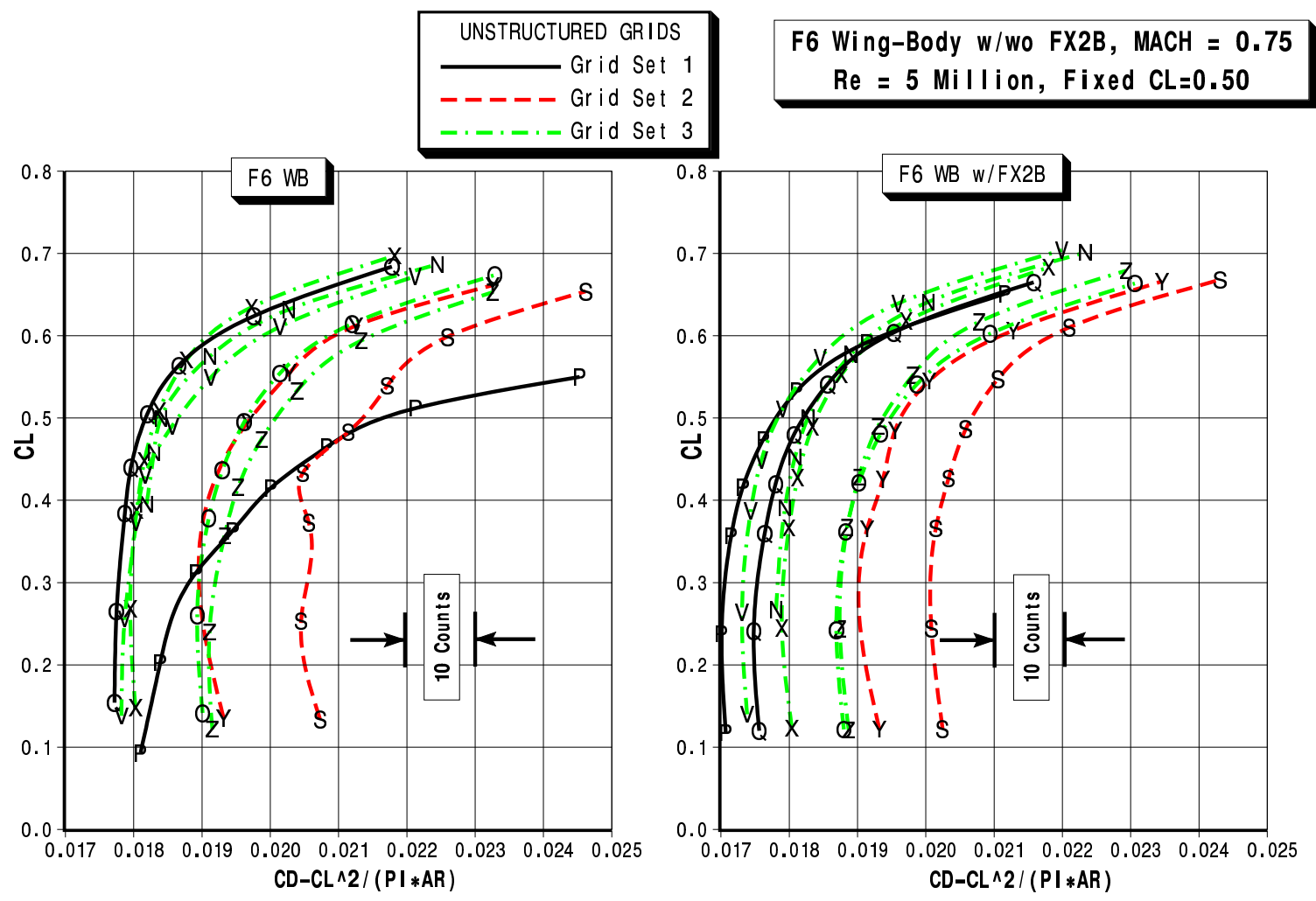

Figure 15. Case 1 Unstructured Mesh Idealized Profile Drag Polars.

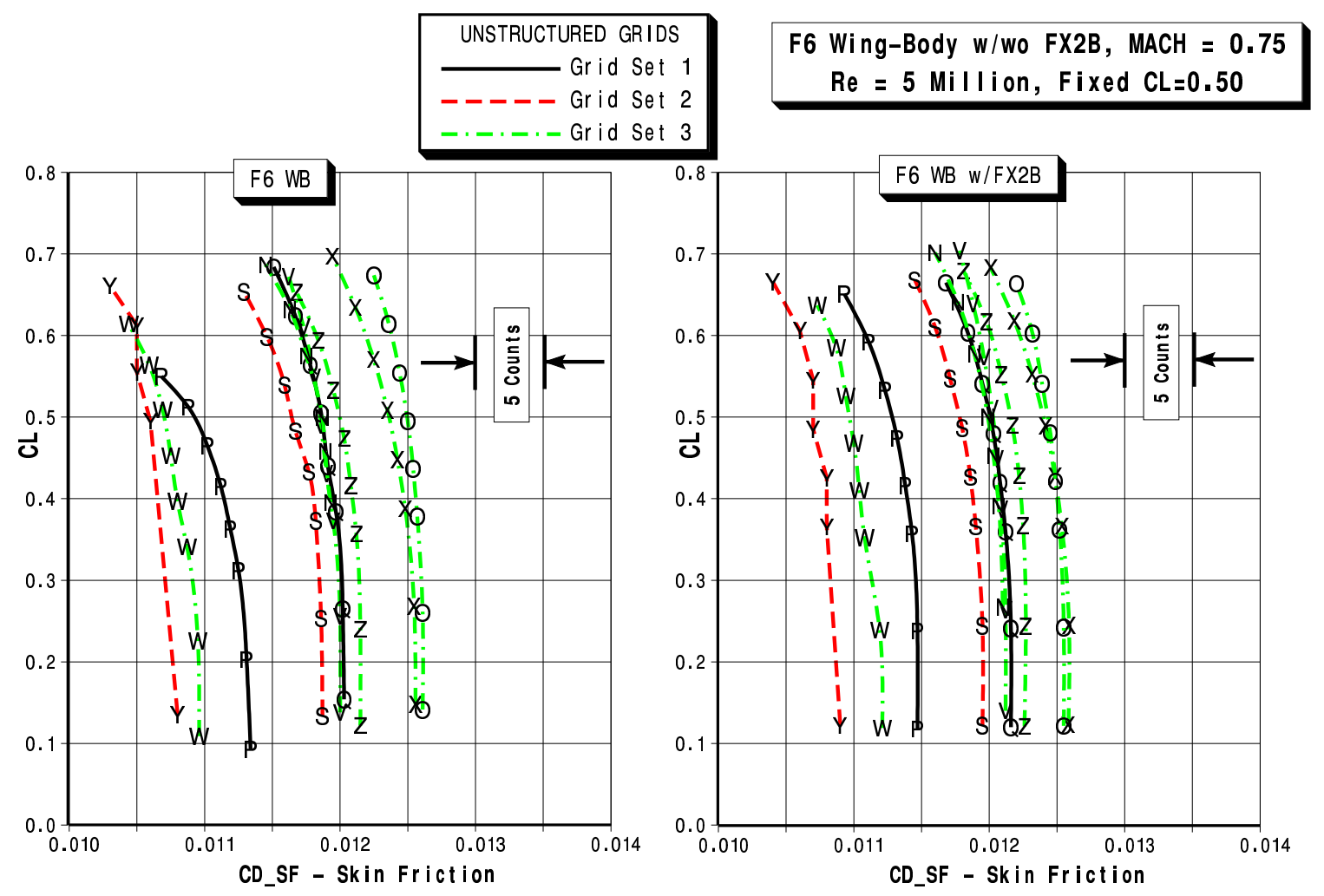

Figure 16. Case 1 Unstructured Mesh Skin-Friction Polars. 


$$
\frac{\pi}{\pi 1}
$$




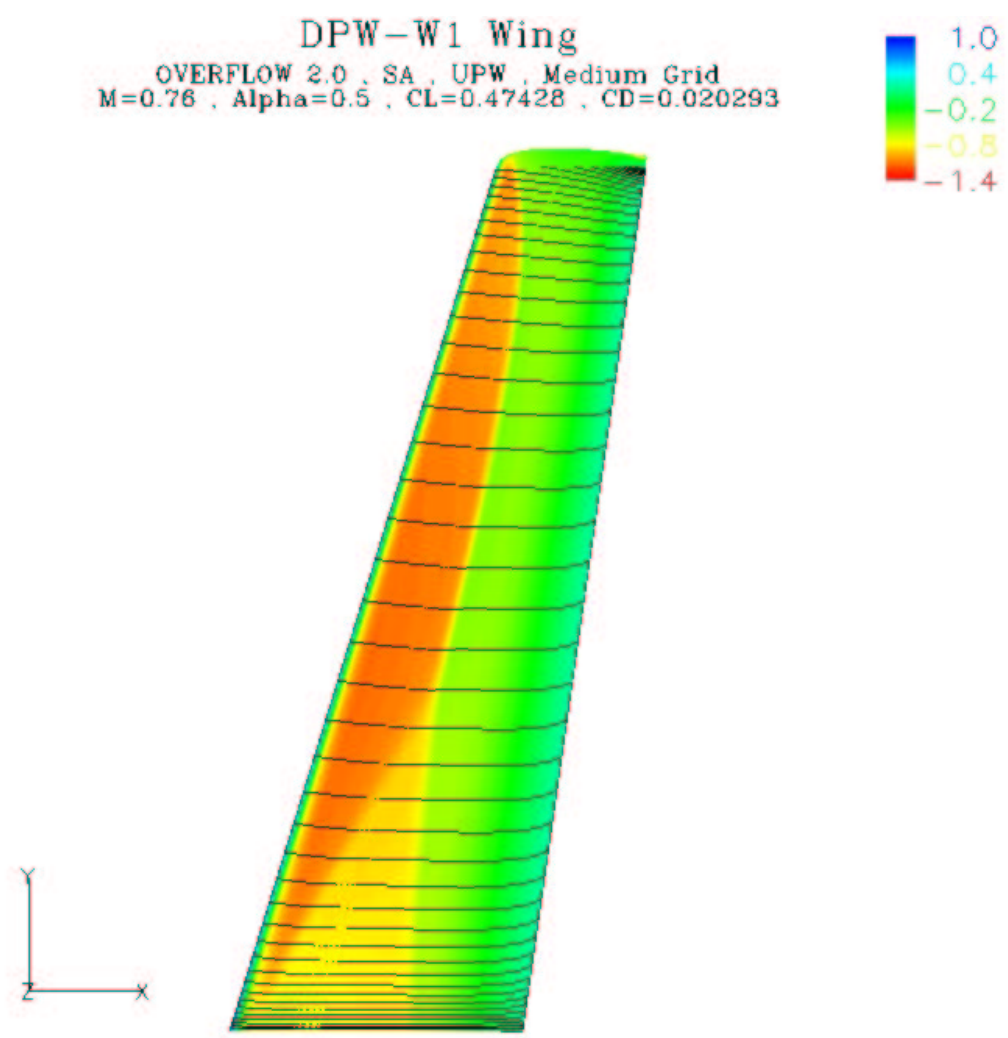

Figure 19. Case 2 W1 Upper-Surface Pressures and Streamlines: $M=0.76, \alpha=0.5^{\circ}, R e=5$ million.

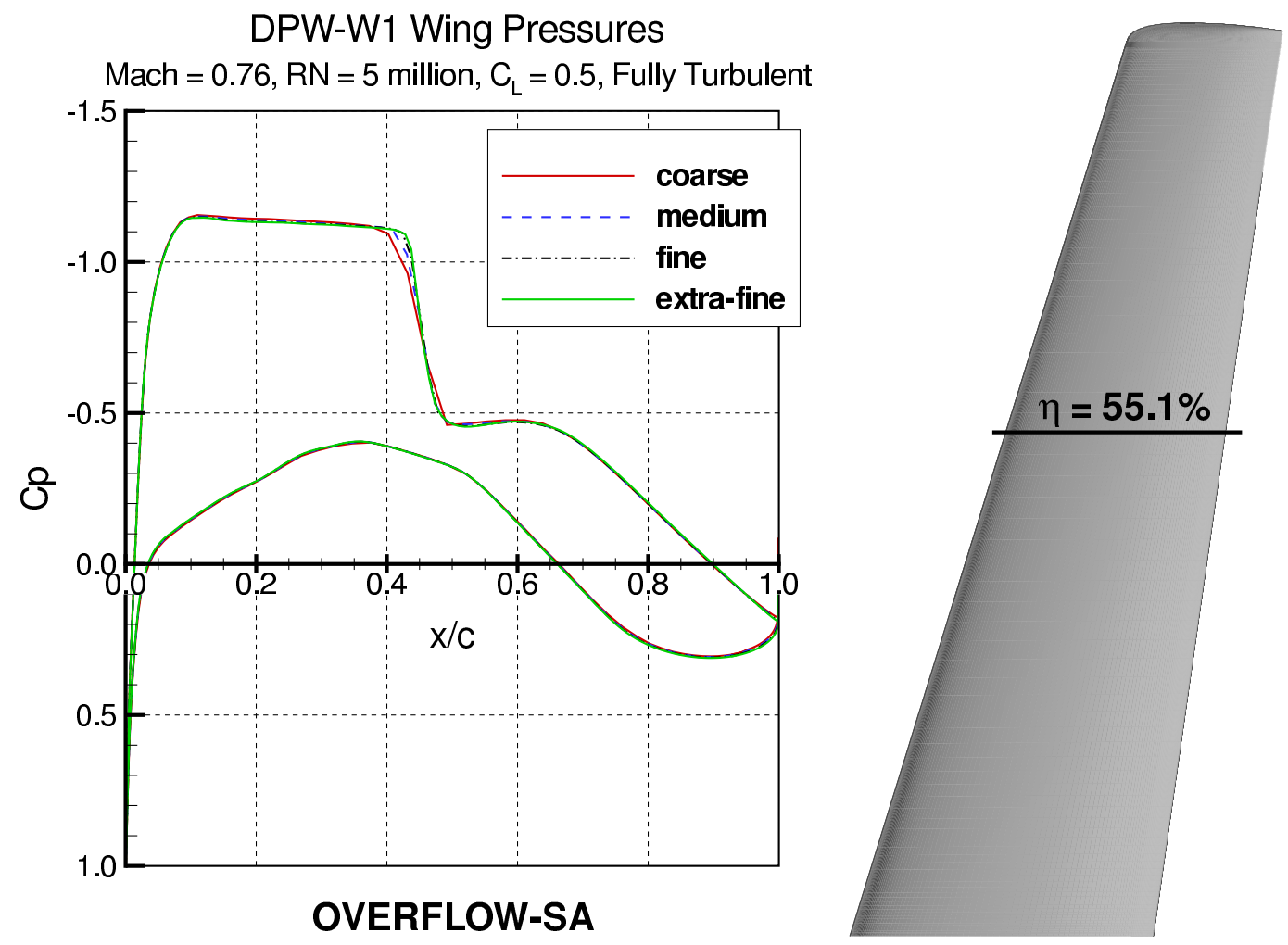

Figure 20. Case 2 W1 Pressure Distributions: $M=0.76, C_{L}=0.5, R e=5$ million. 


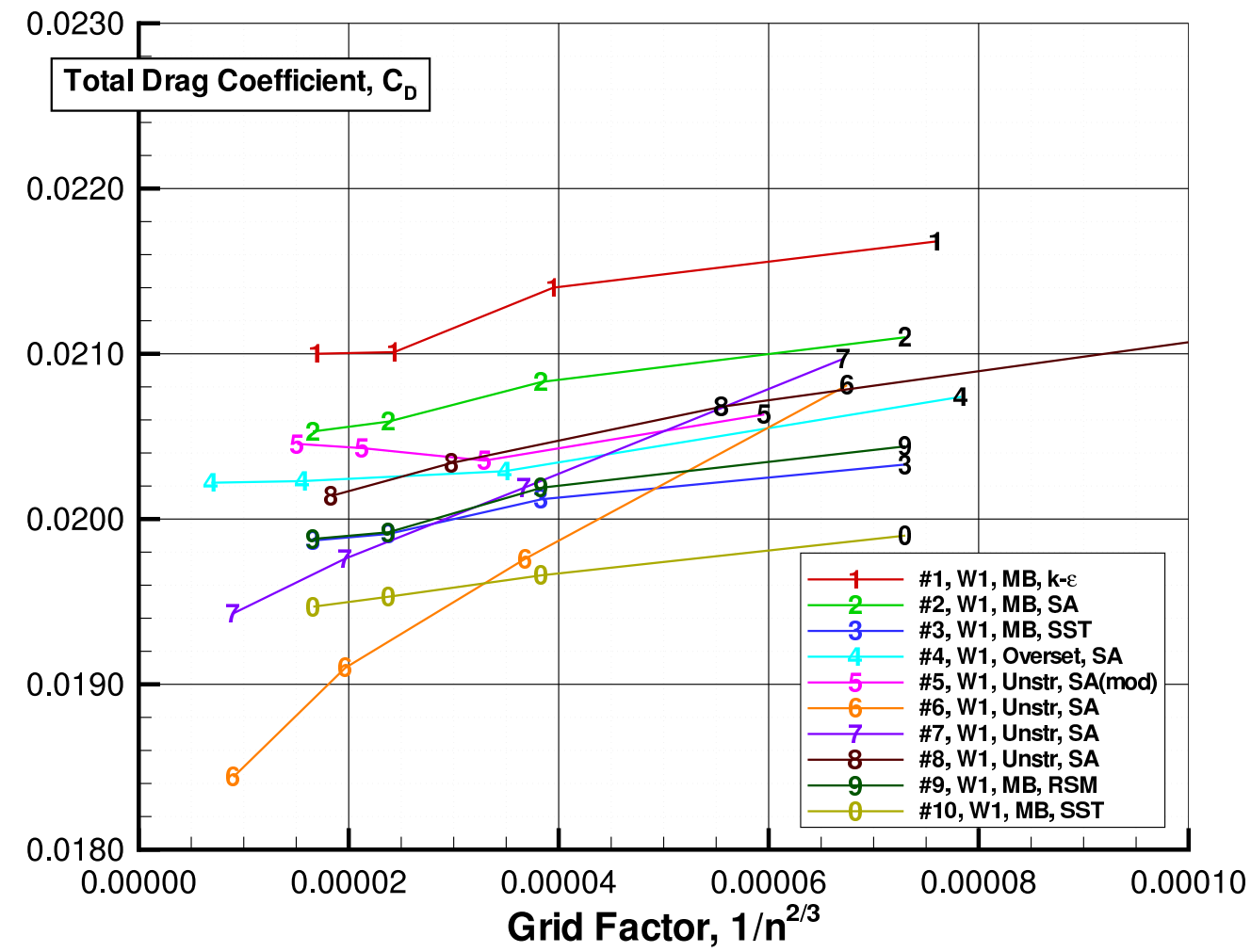

Figure 21. Case 2 Grid Sensitivity on W1 Total Drag: $M=0.76, \alpha=0.5^{\circ}, R e=5$ million .

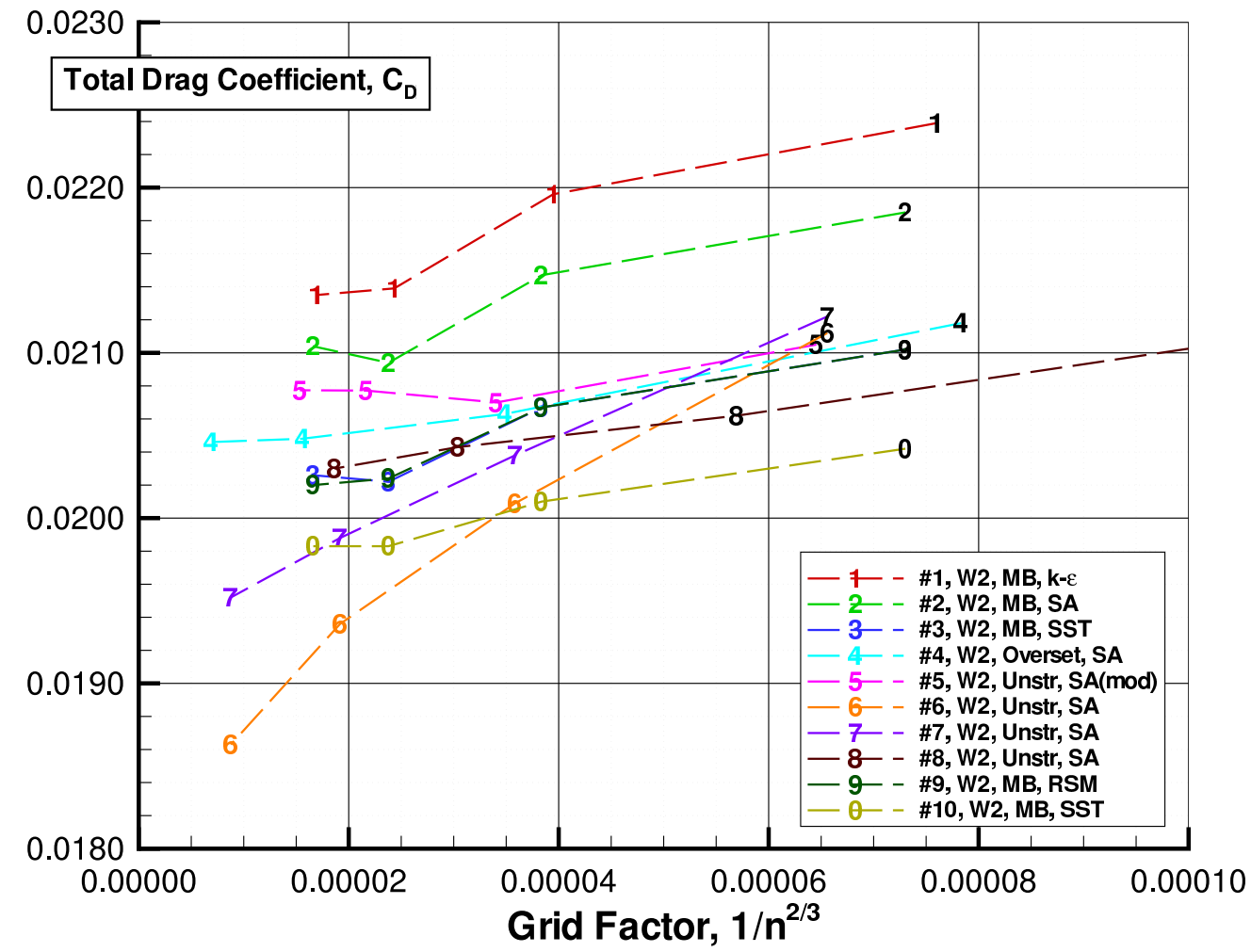

Figure 22. Case 2 Grid Sensitivity on W2 Total Drag: $M=0.76, \alpha=0.5^{\circ}, R e=5$ million. 


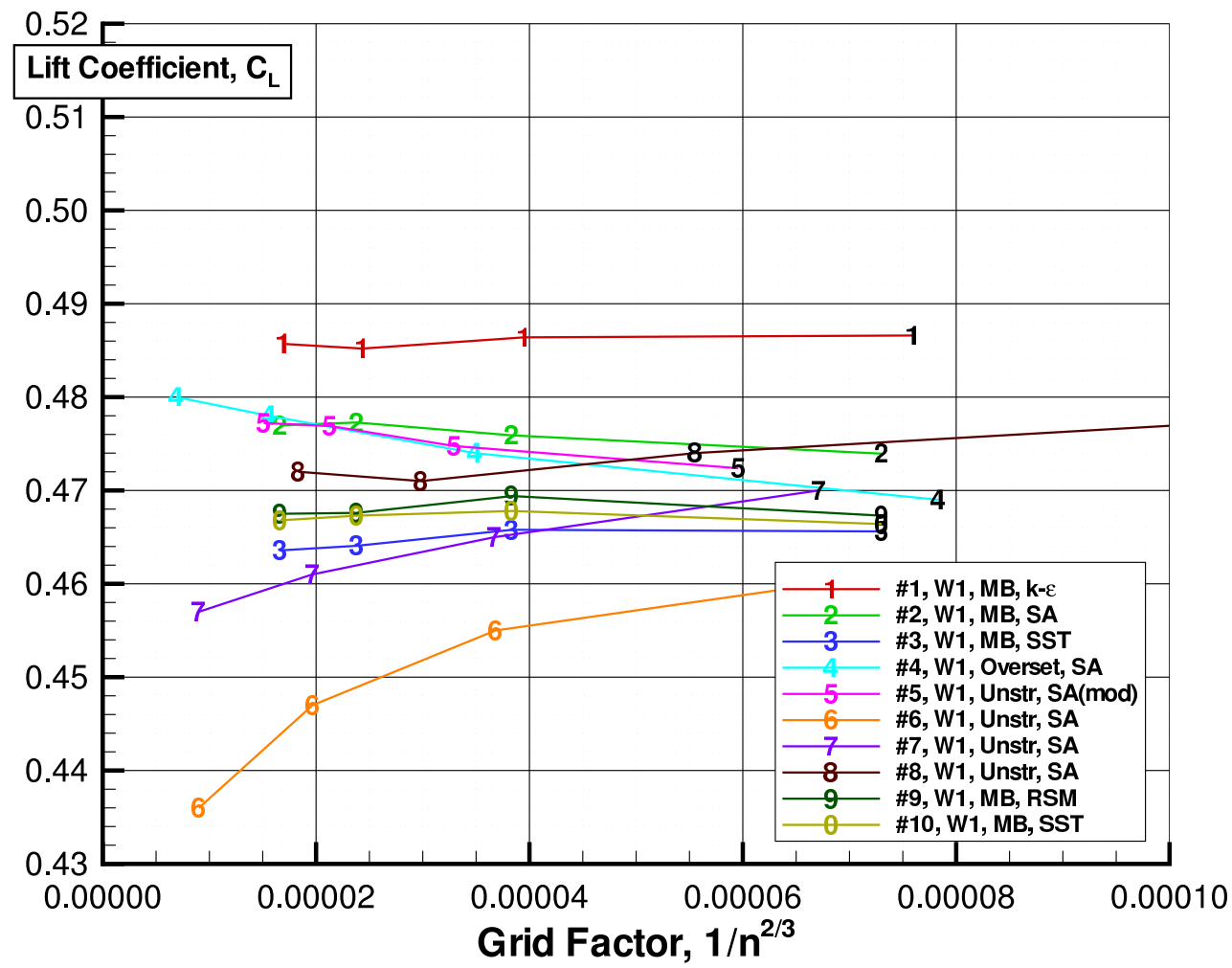

Figure 23. Case 2 Grid Sensitivity on W1 Lift: $M=0.76, \alpha=0.5^{\circ}, R e=5$ million.

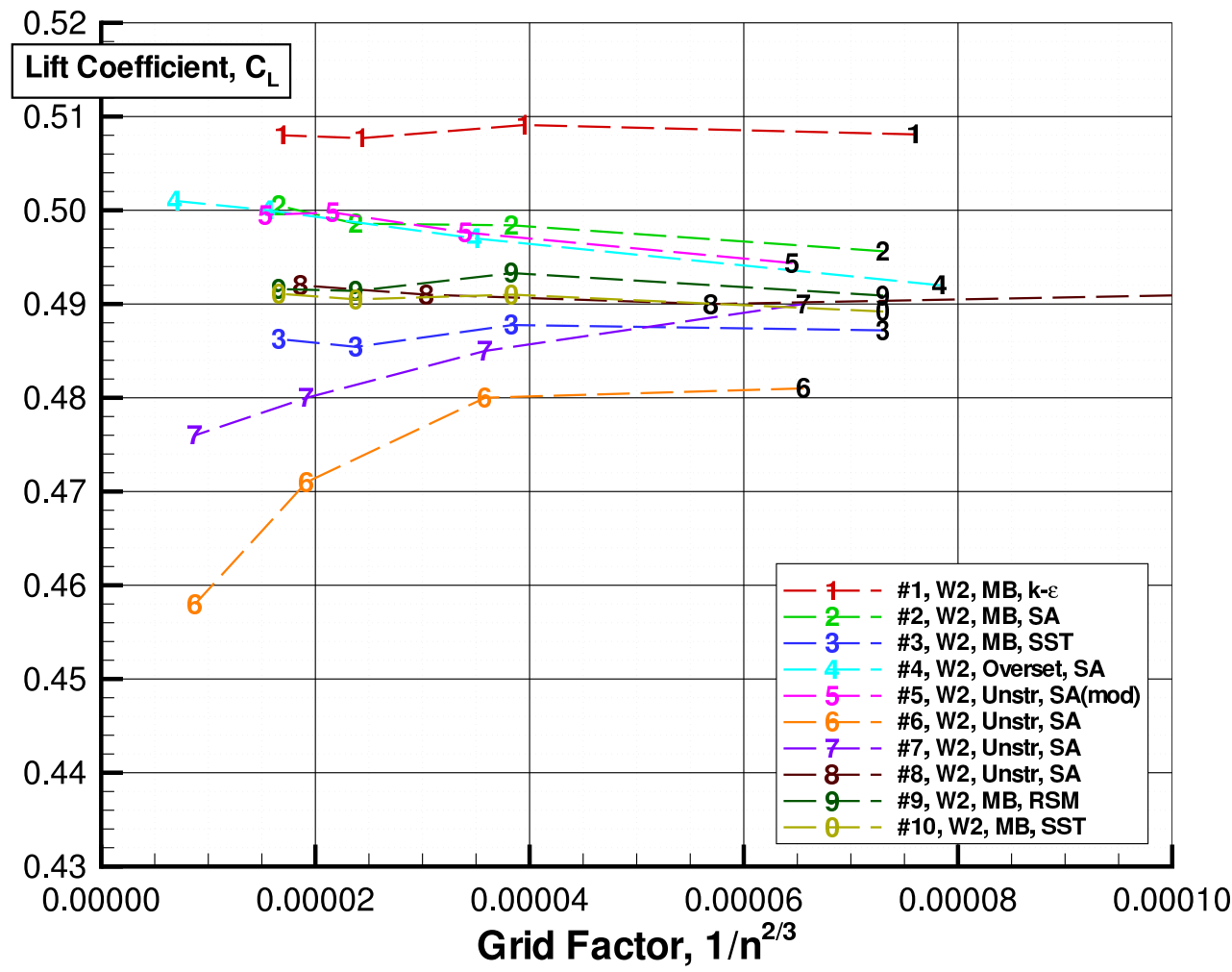

Figure 24. Case 2 Grid Sensitivity on W2 Lift: $M=0.76, \alpha=0.5^{\circ}, R e=5$ million. 


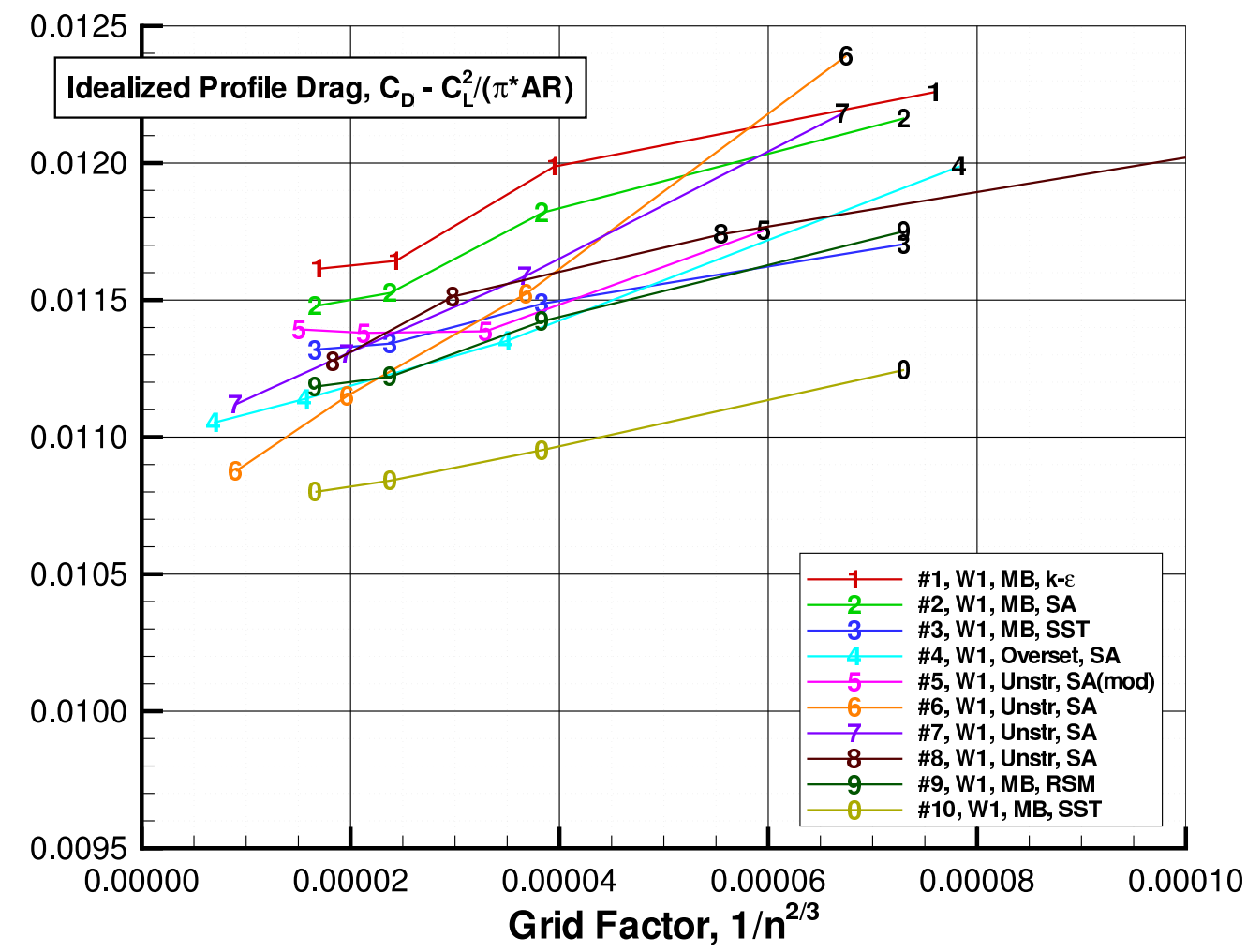

Figure 25. Case 2 Grid Sensitivity on W1 Idealized Profile Drag: $M=0.76, \alpha=0.5^{\circ}, R e=5 \mathrm{million}$.

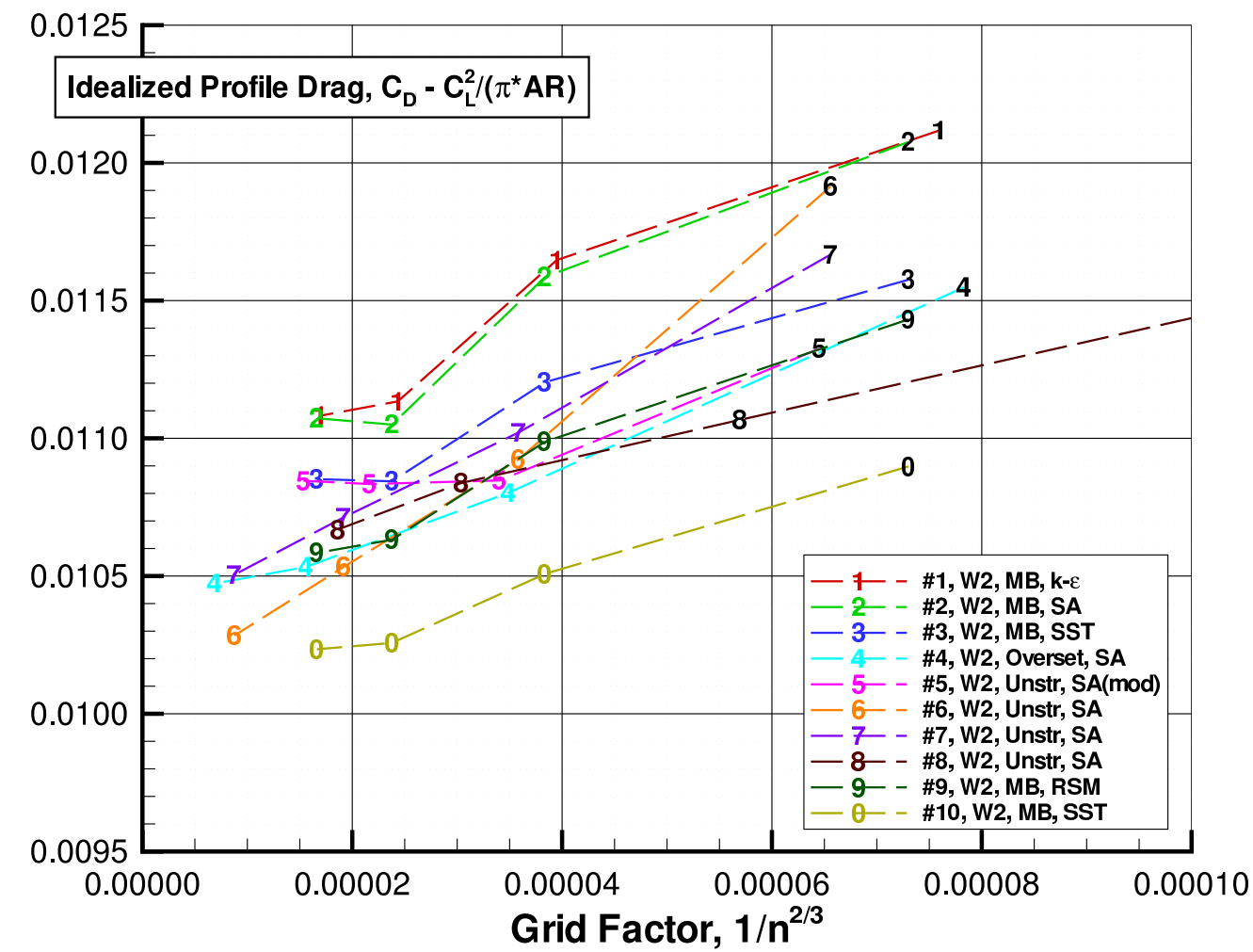

Figure 26. Case 2 Grid Sensitivity on W2 Idealized Proflle Drag: $M=0.76, \alpha=0.5^{\circ}, R e=5 \mathrm{million}$. 


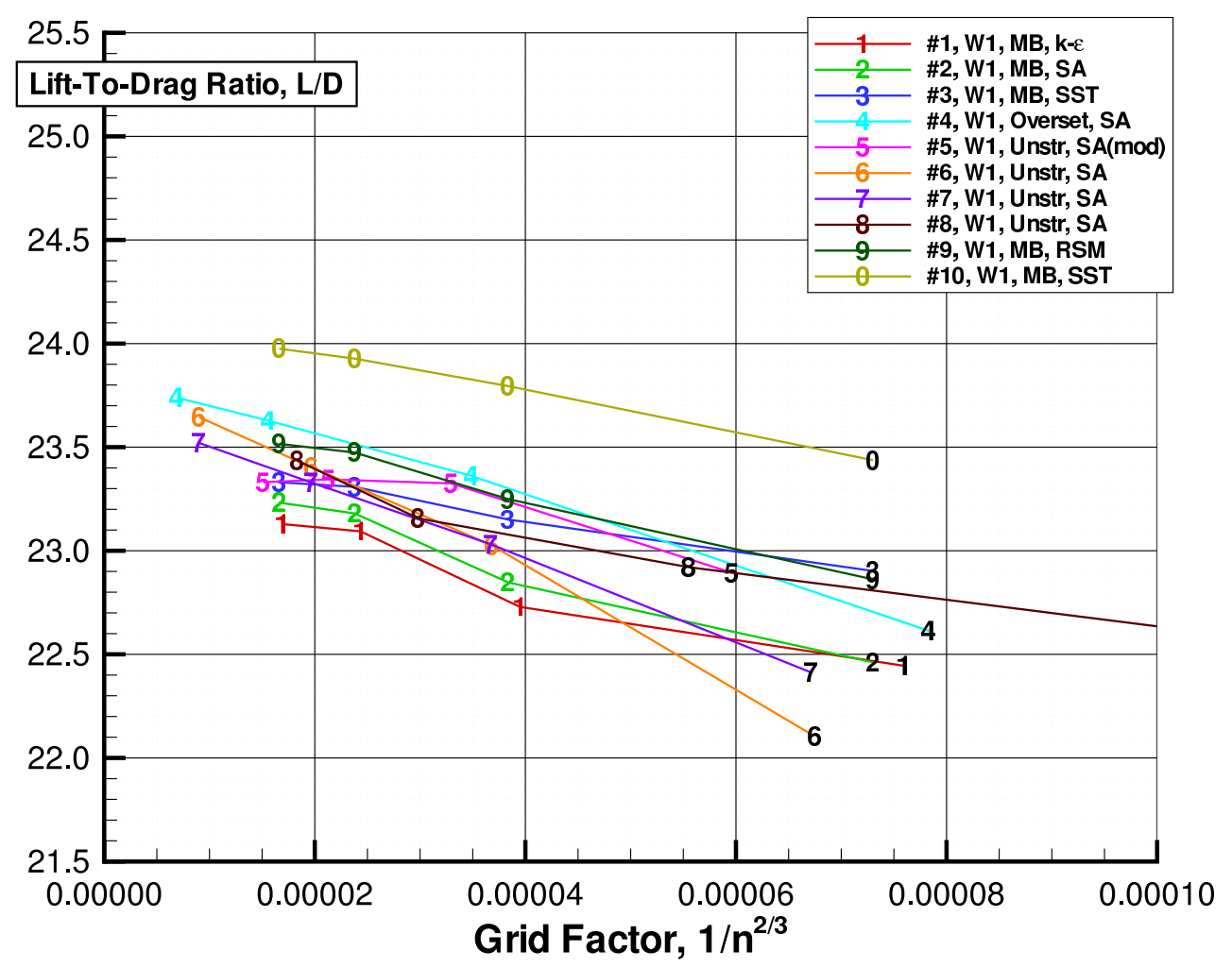

Figure 27. Case 2 Grid Sensitivity on W1 Lift-to-Drag Ratio: $M=0.76, \alpha=0.5^{\circ}$, Re $=5$ million.

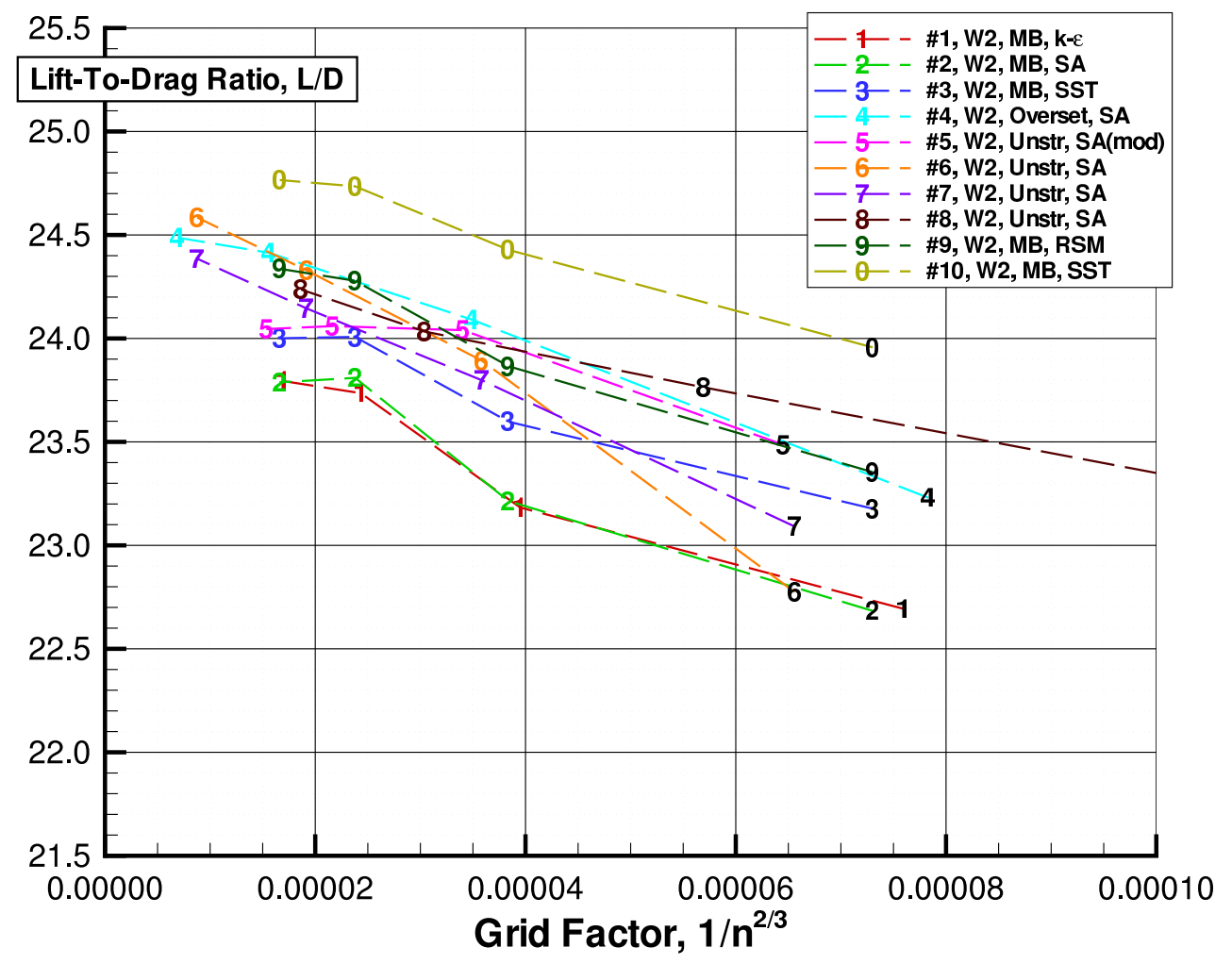

Figure 28. Case 2 Grid Sensitivity on W2 Lift-to-Drag Ratio: $M=0.76, \alpha=0.5^{\circ}, R^{2}=5$ million. 
- $W_{1}=W_{2} \bullet$ Average

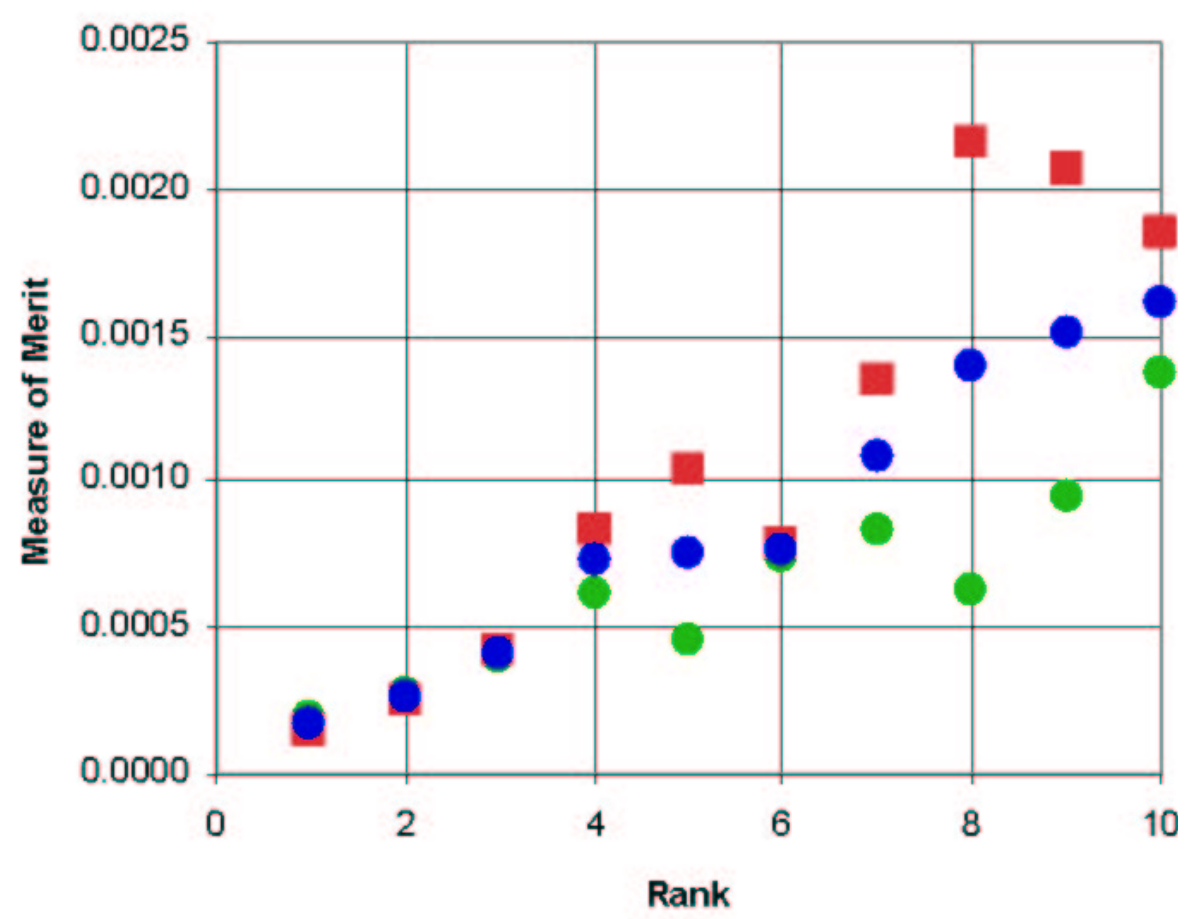

Figure 29. Case 2 Ranked by Measure of Merit.

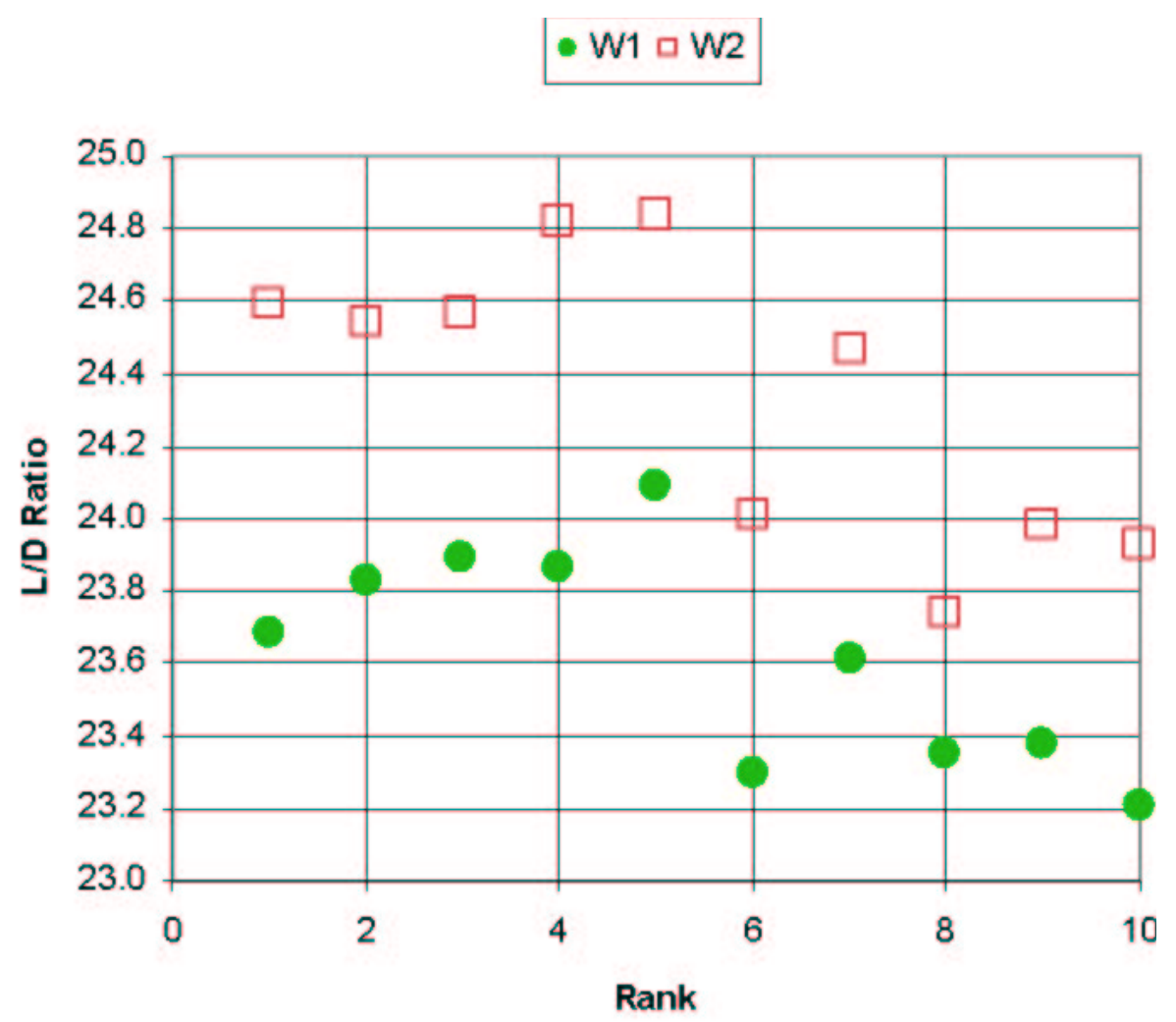

Figure 30. Case 2 Ranked Continuum Lift-to-Drag Ratio. 


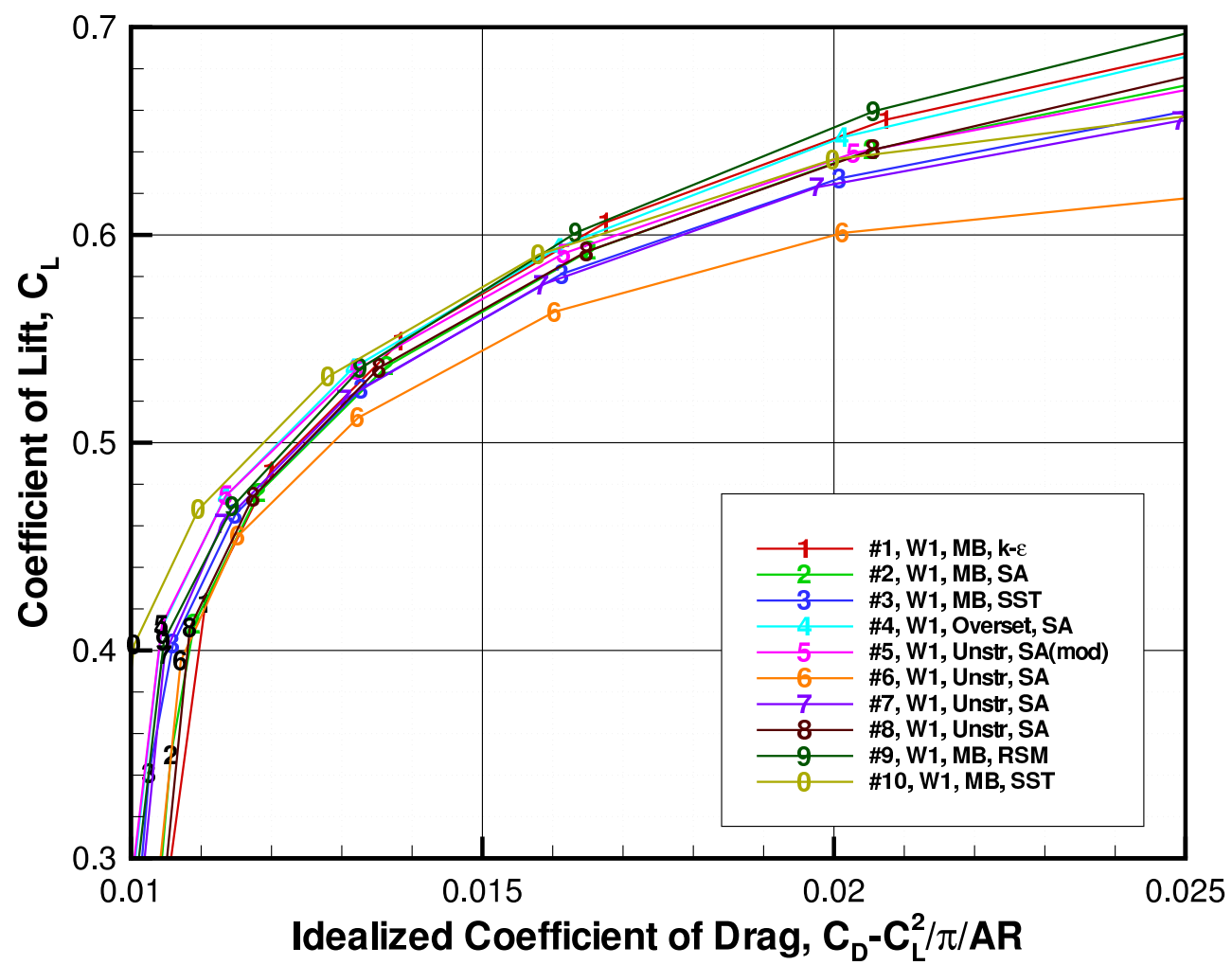

Figure 31. Case 2 W1 Idealized Profile Drag Polar: $M=0.76, R e=5$ million, Medium Mesh.

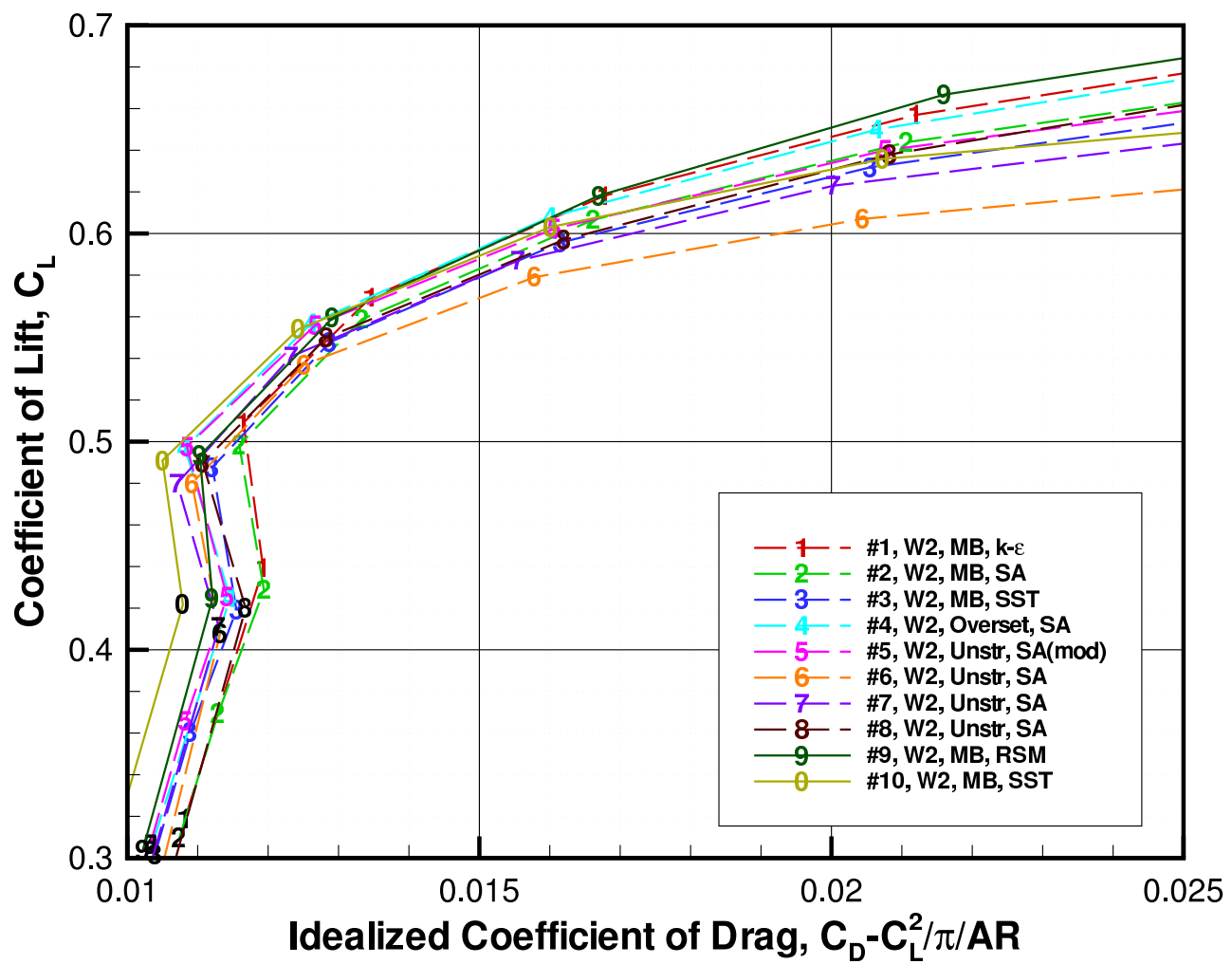

Figure 32. Case 2 W2 Idealized Profle Drag Polar: $M=0.76, R e=5$ million, Medium Mesh. 


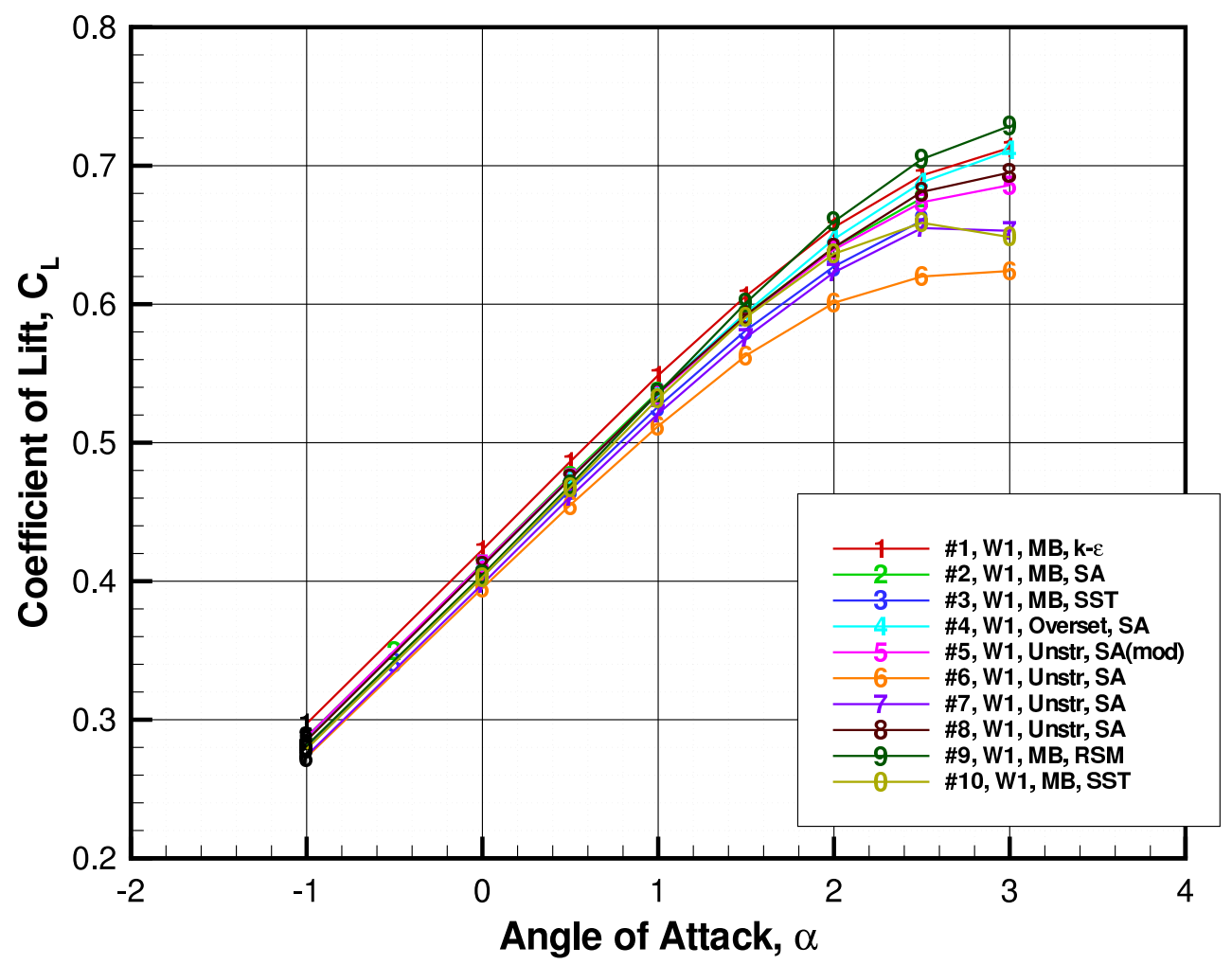

Figure 33. Case 2 W1 $C_{L}-\alpha$ Curves: $M=0.76, R e=5$ million, Medium Mesh.

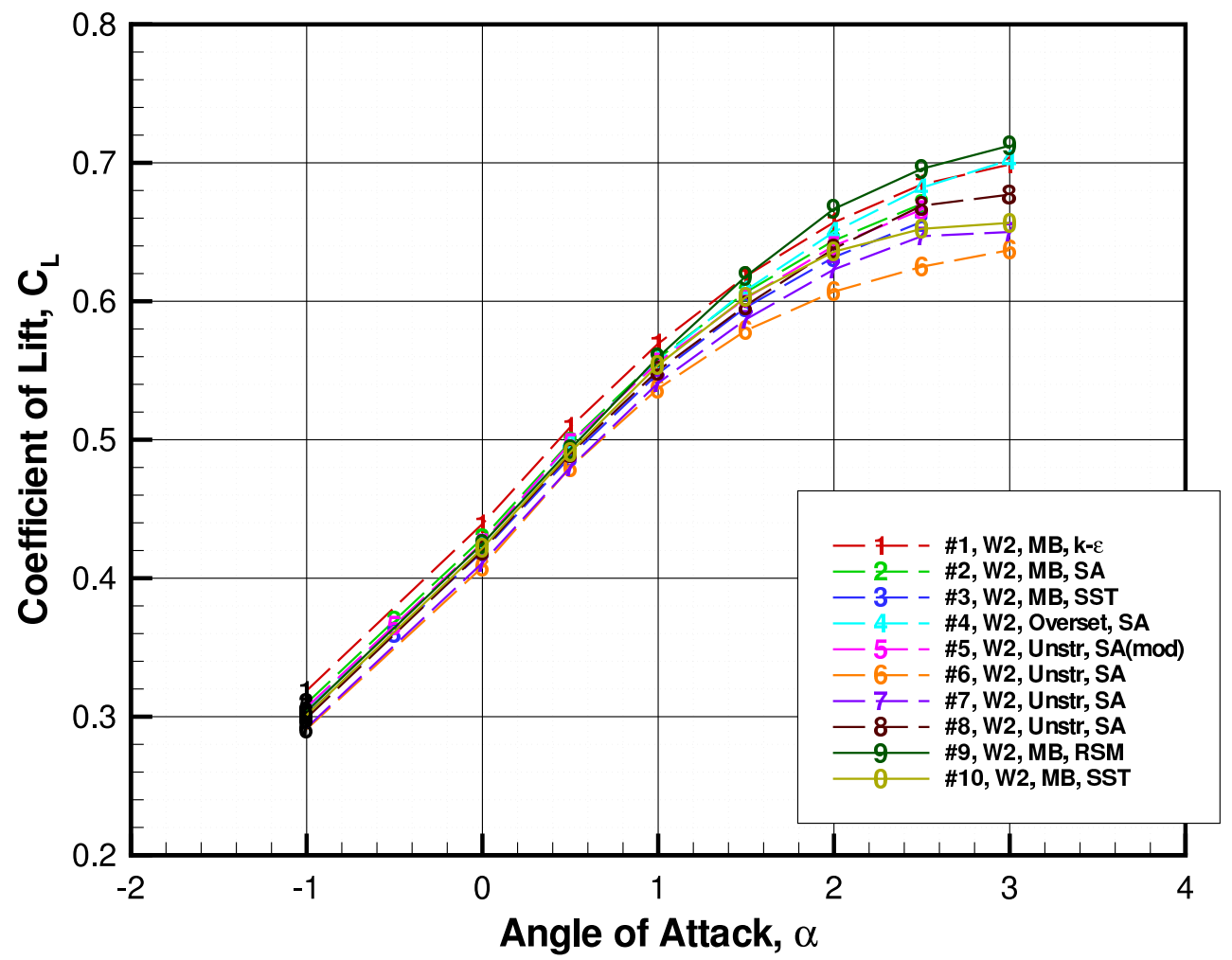

Figure 34. Case 2 W2 $C_{L}-\alpha$ Curves: $M=0.76, R e=5$ million, Medium Mesh. 


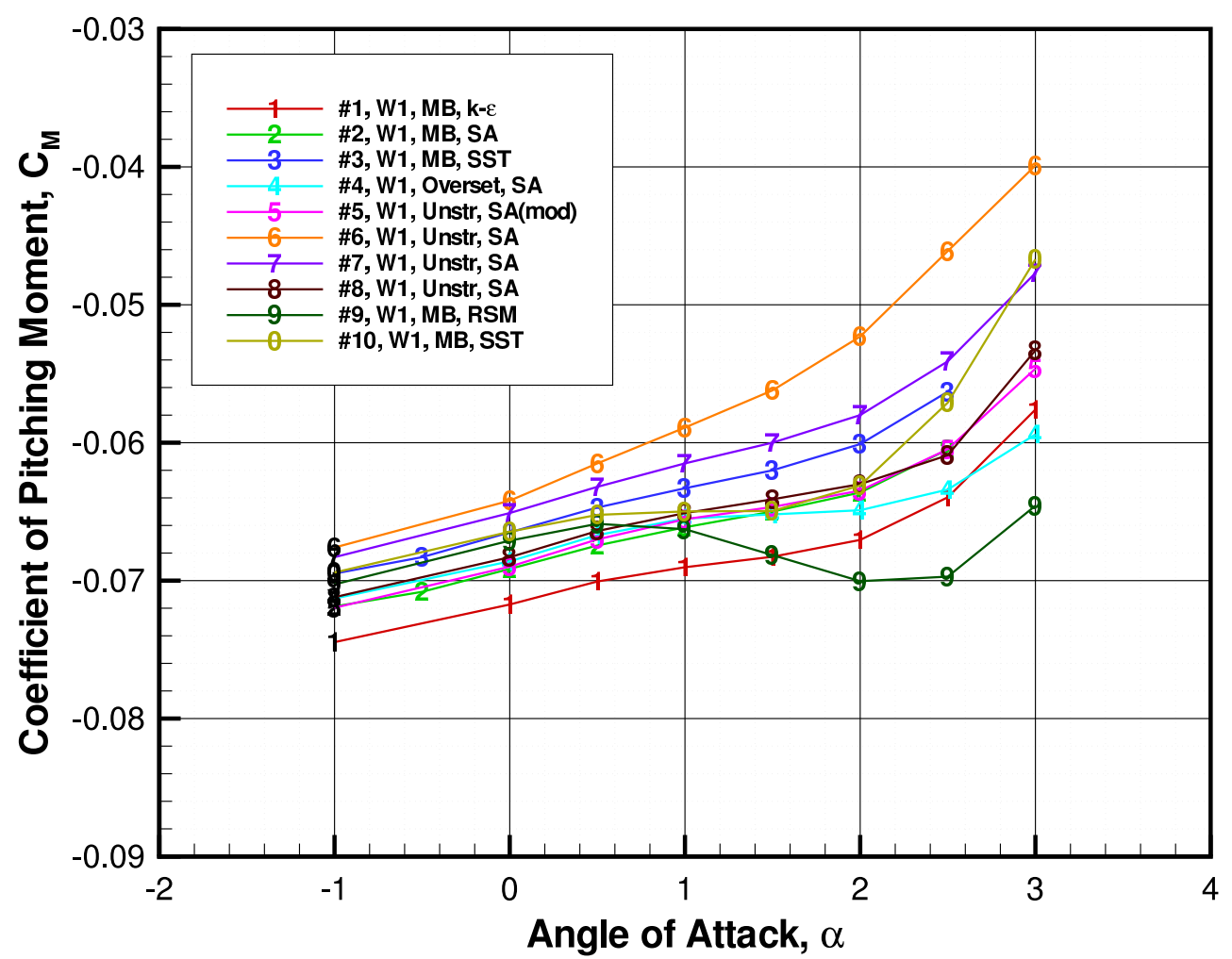

Figure 35. Case 2 W1 $C_{M}-\alpha$ Curves: $M=0.76, R e=5$ million, Medium Mesh.

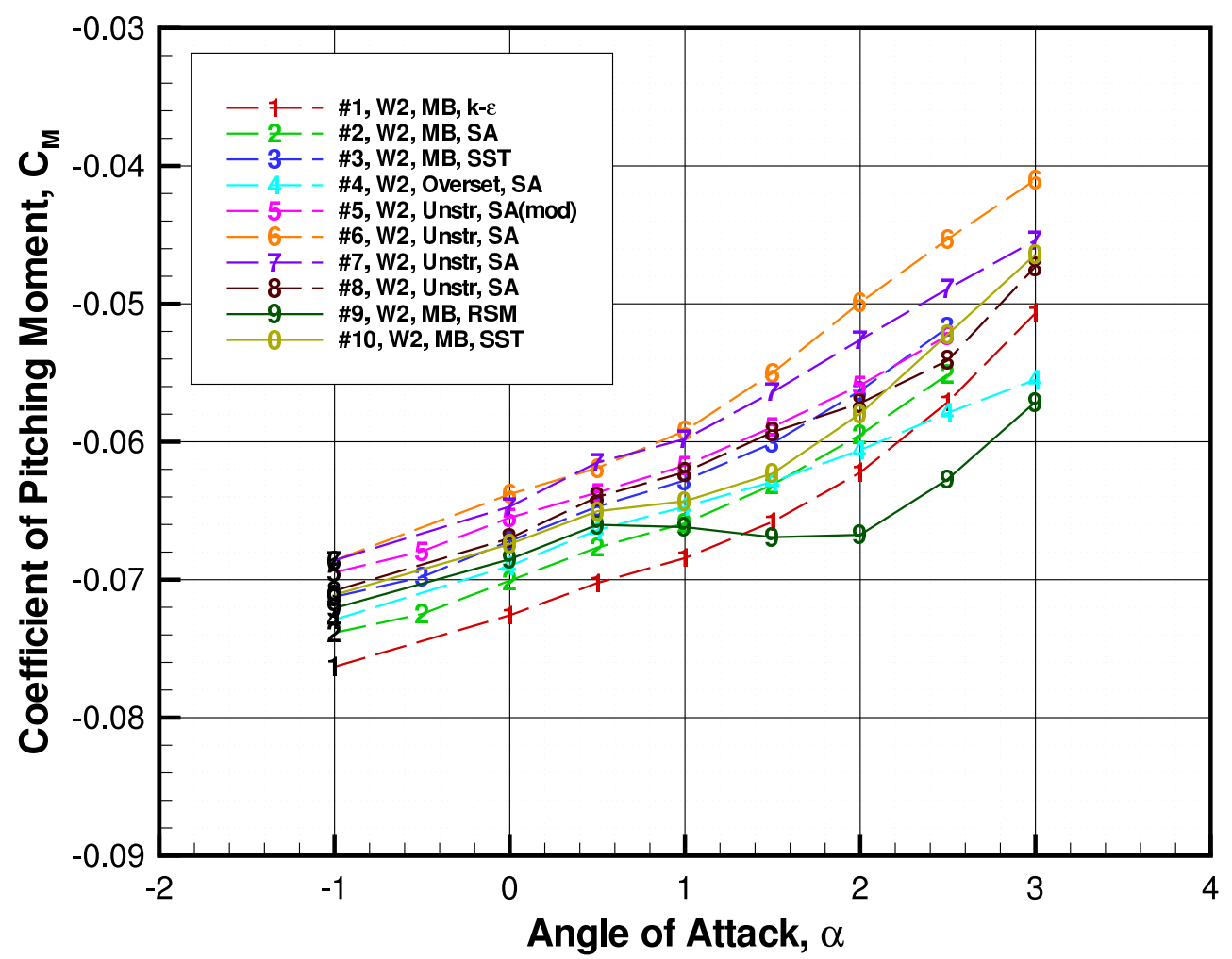

Figure 36. Case 2 W2 $C_{M}-\alpha$ Curves: $M=0.76, R e=5$ million, Medium Mesh. 